Invariantes de variedades determinantais

Nancy Carolina Chachapoyas Siesquén 

SERVIÇO DE PÓS-GRADUAÇ̃̃O DO ICMC-USP

Data de Depósito:

Assinatura:

\title{
Invariantes de variedades determinantais
}

\author{
Nancy Carolina Chachapoyas Siesquén
}

Orientadora: Profa. Dra. Maria Aparecida Soares Ruas

Orientador da instituição conveniada: Prof. Dr. Jean-Paul Brasselet

Tese apresentada ao Instituto de Ciências Matemáticas e de Computação - ICMC-USP, como parte dos requisitos para obtenção do título de Doutor em Ciências - Matemática. VERSÃO REVISADA. 
Ficha catalográfica elaborada pela Biblioteca Prof. Achille Bassi e Seção Técnica de Informática, ICMC/USP, com os dados fornecidos pelo(a) autor(a)

C431i Chachapoyas Siesquen, Nancy Carolina Invariantes de variedades determinantais / Nancy Carolina Chachapoyas Siesquen; orientadora Maria Aparecida Soares Ruas; co-orientador Jean-Paul Brasselet. -- São Carlos, 2014. $82 \mathrm{p}$.

Tese (Doutorado - Programa de Pós-Graduação em Matemática) -- Instituto de Ciências Matemáticas e de Computação, Universidade de São Paulo, 2014.

1. Variedades determinantais. 2. Transformada de Nash. 3. Hiperplano genérico. 4. Seção genérica. 5 . Obstrução de Euler. I. Soares Ruas, Maria Aparecida, orient. II. Brasselet, Jean-Paul, co-orient. III. Título. 


\begin{abstract}
A Matemática é o mais maravilhoso instrumento criado pelo gênio do homem para a descoberta da verdade.

\section{Laisant.}

Going after a dream has a price. But however costly it may be, it is never as high as the price paid by people who didn't live!

Paulo Coelho. 


\section{Agradecimentos}

É com muita satisfação que expresso aqui o mais profundo agradecimento a todos aqueles que tornaram a realização deste trabalho possível.

Em primeiro lugar gostaria de expressar minha gratidão a Deus por seu infinito amor, por todas as alegrias, pela saúde e pela força que me concedeu, para que conseguisse chegar até aqui.

Gostaria antes de mais nada de agradecer aos meus Professores orientadores, Profa. Dra. Maria Aparecida Soares Ruas, pelo apoio nesses momentos em que quase desistia e me sentia perdida, pelo incentivo de continuar, pela confiança desprendida. Ao prof. Dr. Jean-Paul Brasselet, pela assistência durante minha estadia na França, pelo apoio e sugestões realizadas, pelo incentivo de progredir. Agradeço aos dois por acreditar sempre no meu trabalho, pela disponibilidade demonstrada, pela amizade e pela enorme contribuição em todas as fases que levaram à concretização deste trabalho.

Quero expressar aqui meus sinceros agradecimentos a minha mãe, María Yolanda, pelo seu carinho e apoio moral incondicional durante o transcurso da minha vida, sempre acreditando em mim, "te amo mamá". A meu pai, Santiago, pelos ensinamentos em minha infância que me foram muito úteis, só Deus sabe quanta saudade tenho de você. A meus irmãos Jimmy, Sandra, José e Alexis pela compreensão, companherismo e carinho durante estes anos, porque mais que irmãos sempre têm sido meus amigos. Talvez nem saibam a sua importância em minha vida, meus sobrinhos Jhon, Juliana e Estrella. Alegrias incomparáveis ao lado deles. Agradeço muito a toda minha família que apesar de estar longe sempre estão comigo no meu coração.

Gostaria de agradecer aos professores Jawad Snoussi e Juan J. Nuño Ballesteros por aceitar ser os pareceristas desta tese, "rapporteurs", pelos comentários e sugestões. Aos professores Nicolas Dutertre, Marcelo Escudeiro por ser parte da banca. Agradeço muito sua participação!

Agradeço aos professores Lê Dung Trang, Terence Gaffney por algumas sugestões e pelas conversas matemáticas.

Aos professores do ICMC, com os quais muito aprendi. Aos amigos que sempre estiveram presentes Luis Sanchez, Manuel Zuloeta, Thaís Dalbelo, Maria Amelia, Andrea e a todos os amigos com os quais compartilhei conversas durante todo este longo trajeto do meu doutorado, muito obrigada pela amizade e por fazerem minha estadia em São Carlos inesquecível.

A meu amor José Vidarte, pelo apoio e pela compreensão com que sempre me brinda. Por 
viii

dar-me forças para não desistir. Suas palavras sempre chegam na hora certa. Obrigada por estar comigo neste longo caminho.

A FAPESP, Fundação de Amparo à Pesquisa do Estado de São Paulo, processo $n^{0}$ 2010/097361 e 2011/20082-6 pelo apoio financeiro.

Reitero aqui minhas palavras, sendo este um grande sonho, não se pode empreendê-lo sem a ajuda dos que nos cercam e querem bem. Um muito obrigado a todos, por estarem lado a lado nessa caminhada. 

Com amor, a minha mãe Yolanda, a meus irmãos Jimmy, Sandra, José e Alexis.

Ao meu pai Santiago em memória a seu décimo sexto aniversário de estar junto a nosso Criador. 


\section{Resumo}

Neste trabalho estudamos variedades determinantais essencialmente isoladas (EIDS), definidas por W. Èbeling e S. M. Gusel̆n-Zade em [23]. Este tipo de singularidades é uma generalização das singularidades isoladas. A variedade determinantal genérica $M_{m, n}^{t}$ é o subconjunto das matrizes $m \times n$, tais que o posto seja menor que $t$, onde $t \leqslant \min \{n, m\}$. Uma variedade $X \subset \mathbb{C}^{N}$ é determinantal se é definida como a pré-imagem de uma função holomorfa $F: \mathbb{C}^{N} \rightarrow M_{m, n}$, sobre a variedade determinantal genérica $M_{m, n}^{t}$, com a condição $\operatorname{codim} X=\operatorname{codim} M_{m, n}^{t}$.

Uma variedade determinantal tem singularidade isolada se $N \leqslant(n-t+2)(m-t+2)$ e admite suavização se $N<(n-t+2)(m-t+2)$. Trabalhos recentes têm estudado variedades determinantais com singularidade isolada, [35, 31]. O número de Milnor de uma superfície determinantal é investigado em [35, 31, 12]. Para variedades determinantais de dimensões maiores a característica de Euler evanescente é definida em [31, 12].

Neste trabalho estudamos o conjunto de limites de hiperplanos tangentes às variedades determinantais $X^{2} \subset \mathbb{C}^{4} \mathrm{e} X^{3} \subset \mathbb{C}^{5}$ para dar uma caracterização deste conjunto, em que o número de Milnor de sua seção com a superfície no primeiro caso ou a 3-variedade no segundo caso não é mínimo. O primeiro caso foi estudado por Jawad Snoussi em [38].

Provamos também que se $X$ é uma EIDS de dimensão $d$ e $H$ e $H^{\prime}$ são dois hiperplanos fortemente gerais, se $P \subset H$ e $P^{\prime} \subset H^{\prime}$ são planos lineares de codimensão $d-2$ contidos respectivamente em $H$ e $H^{\prime}$, o número de Milnor das superfícies correspondentes $X \cap P$ e $X \cap P^{\prime}$ são iguais. Este resultado foi provado para o caso em que a seção genérica é uma curva em [26].

Estudamos a transformada de Nash de uma EIDS e discutimos condições suficientes para que esta transformada seja suave.

Outro objetivo é estudar a obstrução de Euler de singularidades determinantais essencialmente isoladas. Obtemos fórmulas que relacionam a obstrução de Euler com a característica de Euler evanescente da suavização essencial de suas seções gerais. Estudamos as variedades determinantais com o conjunto singular de dimensão 1 para ilustrar os resultados. 


\section{Résumé}

Dans ce travail nous étudions les variétés determinantales essentiellement isolées (EIDS), qui ont éte définies dans l'article [23]. Ce type de singularité est une généralization de la notion de singularité isolée. La variété determinantale générique est un sous-ensemble des matrices, $m \times n$, tels que le rang est inférieur que $t$, où $t \leqslant \min \{n, m\}$. Une variété $X \subset \mathbb{C}^{N}$ est determinantal si $X$ est définie comme la pré-image d'une fonction holomorphe, $F: \mathbb{C}^{N} \rightarrow M_{m, n}$, sur la variété determinantale générique, $M_{m, n}^{t}$, avec la condition $\operatorname{codim} X=\operatorname{codim} M_{m, n}^{t}$.

Les variétés determinantales ont des singularités isolées si $N \leqslant(n-t+2)(m-t+2)$ et elles admettent un "lissage" si $N<(n-t+2)(m-t+2)$. Certains travaux précédents ont étudié les variétés determinantales avec singularité isolée [35, 31] et ils ont défini le nombre de Milnor d'une surface determinantale. La caractéristique évanescente d'Euler a été definie dans [31].

Nous étudions l'ensemble des hyperplans limites d'hyperplans tangents à une variété determinantale $X^{2} \subset \mathbb{C}^{4}$ et $X^{3} \subset \mathbb{C}^{5}$ pour donner une caractérisation de ces hyperplans, par le fait que le nombre de Milnor de leur section avec la surface dans le premier cas ou la 3- variété dans le deuxième cas n'est pas minimum. Le premier cas a été étudié par Jawad Snoussi dans [38].

Nous montrons également que, si $X$ est une EIDS, de dimension $d$ et $H$ et $H^{\prime}$ sont des hyperplans fortement généraux, si $P \subset H$ et $P^{\prime} \subset H^{\prime}$ sont des plans de codimension $d-2$ contenus respectivement dans $H$ et $H^{\prime}$, les nombres de Milnor des surfaces $X \cap P$ et $X \cap P^{\prime}$ sont égaux. Ce résultat a été démontré dans le cas où la section est une courbe dans [26].

Nous étudions aussi la modification de Nash d'une EIDS et donnons des conditions suffisantes pour que cette transformation soit lisse.

Un autre objectif de notre travail est l'étude de l'obstruction d'Euler des variétés déterminantales essentiellements isolées. Nous obtenons des formules inductives qui relient l'obstruction d'Euler de $X$ à la caractéristique d'Euler évanescente du lissage essentiel de leurs sections génériques. Nous étudions les variétés déterminantales dont l'ensemble singulier est de dimension 1 pour illustrer ce résultat 


\section{Abstract}

In this work, we study the essentially isolated determinantal singularities (EIDS), which have been defined by W. Ėbeling and S. M. Guseĭn-Zade in the article [23]. This type of singularities is a natural generalization of isolated ones. A generic determinantal variety $M_{m, n}^{t}$ is a subset of the space of $m \times n$ matrices, given by matrices of rank less than $t$, where $t \leqslant$ $\min \{n, m\}$. A variety $X \subset \mathbb{C}^{N}$ is determinantal if $X$ is defined as the pre-image of $M_{m, n}^{t}$ by a holomorphic function $F: \mathbb{C}^{N} \rightarrow M_{m, n}$ with the condition $\operatorname{codim} X=\operatorname{codim} M_{m, n}^{t}$.

Determinantal varieties have isolated singularity if $N \leqslant(n-t+2)(m-t+2)$ and they admit smoothing if $N<(n-t+2)(m-t+2)$. Several recent works investigate determinantal variety with isolated singularities. The Milnor number of a surface was defined in [35, 31] and the vanishing Euler characteristic was studied in [31].

In this work we study the set of limits of tangent hyperplanes to determinantal varieties $X^{2} \subset \mathbb{C}^{4}$ and $X^{3} \subset \mathbb{C}^{5}$ to give a characterization of this set by the fact that the Milnor number of its section with the surface in the first case or the 3-dimensional determinantal variety in the second case is not minimum. The first case is studied by Jawad Snoussi in [38].

We also prove that if $X$ is a $d$-dimensional EIDS and $H$ and $H^{\prime}$ are strongly general hyperplans, if $P \subset H$ and $P^{\prime} \subset H^{\prime}$ are linear plans of codimension $d-2$ contained in $H$ and $H^{\prime}$, the Milnor number of the surfaces $X \cap P$ and $X \cap P^{\prime}$ are equal. In the case that the generic section is a curve the result has been proved in [26].

We study the Nash transformation of an EIDS and give sufficient conditions for this transformation to be smooth.

Another aim of our study is the Euler obstruction of essentially isolated determinantal singularities. We obtain inductive formulas associating the Euler obstruction with the vanishing Euler characteristic of the essencial smoothing of their generic sections. We study the determinantal variety with singular set of dimension 1 to illustrate the results. 


\section{Sumário}

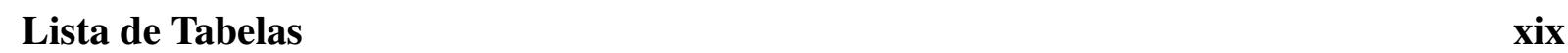

Introdução 1

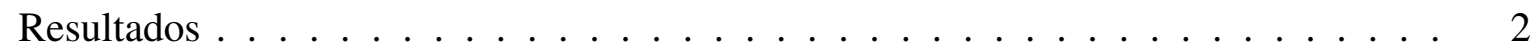

1 Preliminares 5

1.1 Variedades Analíticas $\ldots \ldots \ldots \ldots \ldots$. . . . . . . . . . . . . . . .

1.2 Estratificação de Whitney $\ldots \ldots \ldots \ldots$. . . . . . . . . . . . 6

1.3 Transformação de Nash e o Fibrado de Nash . . . . . . . . . . . . . . . . . . . 6

$1.4 \quad$ Variedades Polares e Multiplicidade Polar . . . . . . . . . . . . . . . . . . . 8

1.5 Fecho Integral de Ideais e de Módulos . . . . . . . . . . . . . . . . . . . 11

1.6 Número de Milnor de uma Curva em $\mathbb{C}^{N} \ldots \ldots \ldots \ldots \ldots$

$\begin{array}{lll}2 & \text { Variedades Determinantais } & 15\end{array}$

$2.1 \quad$ Variedades Determinantais Essencialmente Isoladas . . . . . . . . . . . . . 15

2.2 Singularidades de Matrizes $\ldots \ldots \ldots \ldots \ldots \ldots$. . . . . . . . . . . 18

$2.2 .1 \mathcal{K}$-Equivalência . . . . . . . . . . . . . . . 19

$2.2 .2 \mathrm{~A} \mathcal{G}(n, m)$ - Equivalência . . . . . . . . . . . . 19

$2.2 .3 \mathrm{~A} \mathcal{K}_{V}$ - Equivalência . . . . . . . . . . . . . 21

$2.2 .4 \mathrm{~A} \mathcal{K}_{H}$ - Equivalência . . . . . . . . . . . . . . 22

2.3 Determinação Finita . . . . . . . . . . . . . . . . . . 23

2.4 Variedades Cohen-Macaulay de Codimensão $2 \ldots \ldots$. . . . . . . . 24

3 Topologia de Variedades com Singularidade Isolada 27

3.1 Teoria de Morse . . . . . . . . . . . . . . . . . . . . . . . . . . . 27 
3.2 Funções Definidas em IDS $\ldots \ldots \ldots$

3.3 Fórmula de Tipo Lê-Greuel para Variedades Determinantais $\ldots \ldots \ldots$

4 A Transformada de Nash de Variedades Determinantais 33

$4.1 \quad$ Resoluções de $M_{m, n}^{t} \ldots \ldots \ldots \ldots \ldots \ldots \ldots \ldots \ldots \ldots$

4.2 Transformação de Nash de uma EIDS $\ldots \ldots \ldots \ldots \ldots \ldots \ldots$

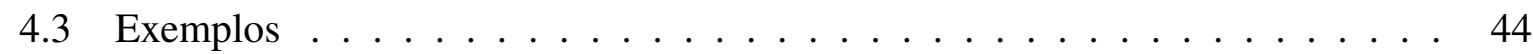

5 Espaço Conormal de Variedades Determinantais 44

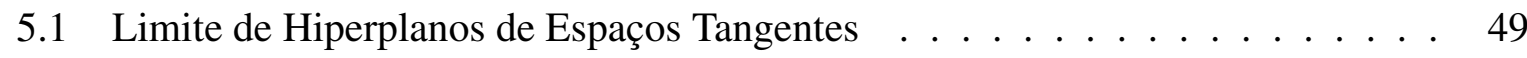

5.2 Minimalidade do Número de Milnor . . . . . . . . . . . . . . . . . . 54

$5.2 .1 \quad$ Hipersuperfícies e Interseções Completas em $\mathbb{C}^{N} \ldots \ldots \ldots \ldots$. . . . 54

$5.2 .2 \quad$ Variedades Determinantais com Singularidade Isolada na Origem . . . 55

5.3 Seções de Variedades Determinantais Essencialmente Isoladas $\ldots . . . . . . \quad 59$

$6 \quad$ Topologia de EIDS

6.1 Indices de Poincaré Hopf de 1-formas sobre EIDS . . . . . . . . . . . . . 61

6.2 Obstrução de Euler Local . . . . . . . . . . . . . . . . . . . . . . . . 65

6.3 Número de Milnor Singular de Seções de Interseções Completas . . . . . . . . 67

6.4 A característica de Euler Evanescente de Variedades Determinantais . . . . . . 69

6.5 Obstrução de Euler de Variedades Determinantais . . . . . . . . . . . . . 71

$6.5 .1 \quad$ Obstrução de Euler de IDS, caso $N<(n-t+2)(m-t+2) \ldots 73$

6.6 Obstrução de Euler para as EIDS, definidas por $F: \mathbb{C}^{N} \rightarrow M_{2,3} \ldots \ldots .73$

6.7 Obstrução de Euler de EIDS, caso $N \geqslant 7 \ldots \ldots \ldots \ldots . \ldots \ldots$

\begin{tabular}{|l|l|}
\hline Referências Bibliográficas & 82
\end{tabular} 


\section{Lista de Tabelas}

6.1 Tabela das equações definindo os divisores livres, $\mathcal{E}$ e as variedades determinantais $\mathcal{D}$. Se $A=\left(A_{i j}\right)$ então $\hat{A}$ denota a matriz obtida eliminando a primeira coluna da matriz $A$ e $A^{(k)}$ denota a submatriz $k \times k$ superior a esquerda de $A .6 \quad 69$

$6.2 \quad$ Ostrução de Euler, $X \subset \mathbb{C}^{6} \ldots \ldots \ldots \ldots \ldots \ldots$. . . . . . . . . . . . .

$6.3 \quad$ Obstrução de Euler, $X \subset \mathbb{C}^{6} . \ldots \ldots \ldots \ldots \ldots \ldots$ 


\section{Introdução}

Um tipo de variedade analítica que pode ser considerado uma geralização para interseções completas com singularidade isolada (ICIS) é a variedade com singularidade determinantal essencialmente isolada (EIDS). Estas variedades foram definidas por W. Èbeling e S. M. Guseĭn-Zade em [23]. As variedades determinantais $X \subset \mathbb{C}^{N}$ são definidas por uma função $F: \mathbb{C}^{N} \rightarrow M_{m, n}$ holomorfa tal que $X=F^{-1}\left(M_{m, n}^{t}\right)$ e que satisfazem certas condições sobre a codimensão de $X$, em que $M_{m, n}^{t}$ é o subconjunto das matrizes $m \times n$ com posto menor do que $t$, chamada variedade determinantal genérica.

Uma EIDS satisfaz condições de transversalidade da $F$ sobre os estratos de $M_{m, n}^{t}$. Nem toda EIDS tem singularidade isolada. Temos singularidade determinantal isolada (IDS) se $N \leqslant$ $(m-t+2)(n-t+2)$. Trabalhos recentes, como por exemplo [31, 35], têm estudado IDS que admitem suavização $(N<(m-t+2)(n-t+2))$. A topologia da fibra genérica da suavização é um invariante da variedade e então se pode definir a característica de Euler evanescente em termos da característica de Euler da fibra genérica, $\nu(X)=(-1)^{d}\left(\chi\left(X_{t}\right)-1\right)$.

No caso de superfícies determinantais com singularidade isolada em $\mathbb{C}^{N}$, o primeiro número de Betti $b_{1}\left(X_{t}\right)$ é nulo [40], então podemos definir o número de Milnor de $X, \mu(X)=b_{2}\left(X_{t}\right)$. Miriam Pereira e Maria Ruas [35], Bruna Oréfice, João Tomazella e Juan Nuño Ballesteros [31] demonstraram uma fórmula de tipo Lê-Greuel em termos do número de Milnor da superfície determinantal ou característica de Euler evanescente.

Neste trabalho desenvolvemos o estudo de seções genéricas de IDS, dadas pela interseção da IDS $X$ com um hiperplano geral, isto é um hiperplano que não é limite de hiperplanos tangentes à variedade $X$. Estudamos superfícies determinantais em $\mathbb{C}^{4}$ e 3 - variedades em $\mathbb{C}^{5}$ e damos uma caracterização das seções genéricas em termos da minimalidade do número de Milnor. $\mathrm{O}$ caso de superfícies determinantais segue do trabalho de Jawad Snoussi [38], pois toda variedade determinantal com singularidade isolada é normal.

Estudamos também seções genéricas de uma EIDS $X \subset \mathbb{C}^{N}$, dadas pela interseção de uma EIDS e $P$-planos que estão contidos num hiperplano fortemente geral, com a condição que essa 
interseção seja uma superfície determinantal (IDS). Sobre algumas condições sobre $N$ podemos garantir que $X \cap P$ admite suavização. Provamos uma generalização do resultado de Lê Dung Trang em [26], obtendo a igualdade do número de Milnor de duas seções gerais de uma EIDS.

No Capítulo 1, relembramos algumas definições e resultados básicos tais como germes de variedades analíticais, estratificação de Whitney, transformada de Nash de uma variedade analítica, variedades polares, fecho integral de módulos e número de Milnor de curvas espaciais.

No Capítulo 2, definimos as variedades essencialmente isoladas (EIDS), suavização essencial de uma EIDS, estudamos diferentes grupos de equivalência que agem sobre o conjunto de funções $F: \mathbb{C}^{N} \rightarrow M_{m, n}$ e relacionamos as EIDS com a teoria de singularidades de matrizes.

No capítulo 3, é estudada a topologia de variedades com singularidade isolada. A fórmula de tipo Lê-Greuel para singularidades determinantais isoladas (IDS) é apresentada.

A transformada de Nash da variedade determinantal genérica $M_{m, n}^{t}$ é suave, no capítulo 4, fazemos um estudo detalhado deste resultado, usando as diferentes resoluções da variedade $M_{m, n}^{t}$. Calculamos o espaço tangente em um ponto regular de $M_{m, n}^{t}$. Provamos em que condições a transformada de Nash de uma EIDS é suave. Concluimos este capítulo dando exemplos de transformada de Nash.

Os resultados obtidos no capítulo 5, são geralizações dos trabalhos de Jawad Snoussi [38] e Lê Dung Trang [26]. Aqui usamos as seções gerais da EIDS, dadas pela interseção de hiperplanos gerais e a variedade EIDS. Provamos que um hiperplano é geral a uma variedade $X \subset \mathbb{C}^{N}$ 3-dimensional se somente se $\mu(X \cap H)$ e $\mu\left(X \cap H \cap H^{\prime}\right)$ são mínimos.

No Capítulo 6, estudamos a topologia de EIDS. Definimos a obstrução de Euler e fornecemos uma fórmula para o cálculo da obstrução de Euler de EIDS que admitem 3 estratos, com a condição que o conjunto singular é uma ICIS. Para variedades determinantais definidas por $F: \mathbb{C}^{N} \rightarrow M_{2,3}, N \geqslant 7$ e de coposto 1 , mostramos que este número é constante e igual a 2.

\section{Resultados}

Os resultados mais relevantes deste trabalho são enunciados nesta seção. As provas são encontradas nos Capítulos 4, 5, 6.

O seguinte resultado dá uma condição para que a transformada de Nash de uma EIDS seja suave.

Proposição 0.1. (Proposição 4.7) Seja $X=F^{-1}\left(M_{m, n}^{t}\right) \subset \mathbb{C}^{N}$ uma EIDS, definida pela função $F: \mathbb{C}^{N} \rightarrow M_{m, n}$. Se $F$ é transversal aos limites de espaços tangentes da variedade $M_{m, n}^{t}$ então a transformada de Nash de $X$ denotado por $\widehat{X}$, é suave.

Dizemos que um hiperplano $H \subset \mathbb{C}^{N}$ é geral a $X$ no ponto $x$ se $H$ não é limite de hiperplanos tangentes a $X$ em $x$. Se $H$ for transversal a todos os estratos da estratificação de $X$ numa 
vizinhança do ponto $x$ dizemos que $H$ é fortemente geral.

Proposição 0.2. (Proposição 5.9) Seja $X \subset \mathbb{C}^{N}$ uma variedade determinantal d-dimensional com singularidade isolada na origem e $H$ um hiperplano geral com relação a $X$, então $X \cap H \subset$ $\mathbb{C}^{N-1}$ é uma variedade determinantal $d-1$ dimensional com singularidade isolada na origem.

Teorema 0.3. (Teorema 5.12) Seja $(X, 0) \subset\left(\mathbb{C}^{N}, 0\right)$ uma EIDS, de tipo $(n, m, t)$. Se $H \subset \mathbb{C}^{N}$ é um hiperplano fortemente geral a $X$ na origem, então $X \cap H$ é uma EIDS em $\mathbb{C}^{N-1}$ de dimensão $d-1$ e do mesmo tipo.

Teorema 0.4. (Teorema 5.21) Seja $X \subset \mathbb{C}^{N}$ uma variedade determinantal de dimensão 3 com singularidade isolada, e seja $H$ um hiperplano em $\mathbb{C}^{N}$ dado pelo núcleo da projeção linear $p: \mathbb{C}^{N} \rightarrow \mathbb{C}$. Suponha que $X \cap H$ tem singularidade isolada na origem, então as seguintes condições são equivalentes:

a) pé geral a $X$ na origem

b) $\mu(X \cap H)$ é mínimo e $\mu\left(X \cap H \cap H^{\prime}\right)$ é mínimo para todo $H^{\prime}$ geral a $X$ e a $X \cap H$.

Teorema 0.5. (Teorema 5.24) Seja $X^{d} \subset \mathbb{C}^{N}$ uma EIDS de tipo $(m, n, t)$ tal que codim $X<$ $(m-t+2)(n-t+2)-2$. Sejam $H$ e $H^{\prime}$ hiperplanos de $\mathbb{C}^{N}$ fortemente gerais para $(X, 0) n a$ origem. Então $H$ e $H^{\prime}$ contêm respetivamente $P$ e $P^{\prime}$ tais que $\operatorname{codim} P=\operatorname{codim} P^{\prime}=d-2$, para os quais as superfícies determinantais $X \cap P$ e $X \cap P^{\prime}$ satisfazem as seguintes condições

a) têm singularidade isolada

b) admitem suavizações

c) $\mu(X \cap P)=\mu\left(X \cap P^{\prime}\right)$.

Proposição 0.6. (Proposição 6.36) Seja $X=F^{-1}\left(M_{m, n}^{t}\right)$ uma EIDS, definida pela função $F: \mathbb{C}^{N} \rightarrow M_{m, n}$, se $N<(n-t+3)(m-t+3)$ e o conjunto singular, $\Sigma X$, é uma ICIS, então a obstrução de Euler de $X$ na origem é dado por:

$$
E u_{0}(X)=\left((-1)^{\operatorname{dim}\left(\Sigma X \cap l^{-1}(0)\right)} \mu\left(\Sigma X \cap l^{-1}(0)\right)+1\right)\left(\chi\left(L_{V_{1}}\right)-1\right)+\tilde{\chi}\left(X \cap l^{-1}(0) \cap B_{\epsilon}\right)+1,
$$

onde l $: \mathbb{C}^{N} \rightarrow \mathbb{C}$ é uma projeção linear genérica, $L_{V_{1}}$ é o link complexo do estrato $V_{1}$ em $X$ e $B_{\epsilon}$ é a bola de raio $\epsilon$ em $\mathbb{C}^{N}$.

Teorema 0.7. (Teorema 6.41) Seja $X \subset \mathbb{C}^{N}$ uma EIDS, definida pela função $F: \mathbb{C}^{N} \rightarrow M_{2,3}$, com $N \geqslant 7$. Então

$$
E u_{0}(X)=(-1)^{N-7} \mu(\Sigma(X \cap H))+\tilde{\chi}(X \cap H)+2
$$

onde $\tilde{\chi}(X)=\chi\left(F_{s}^{-1}\left(M_{2,3}^{2}\right)\right)-1, F_{s}$ é uma estabilização de $F$. 
Corolário 0.8. (Corolário 6.42) Com as hipóteses do Teorema 0.7, se F é de coposto igual a 1 , então

$$
E u_{0}(X)=2
$$




\section{Preliminares}

Neste capítulo apresentamos as definições e principais resultados que serão usados para o desenvolvimento deste trabalho. Começamos definindo variedades analíticas, estratificação de Whitney, transformação de Nash de variedades analíticas. O nosso objeto de estudo são as variedades determinantais que serão definidas no próximo capítulo. As principais referências para este capítulo são [8, 24, 27, 21].

\subsection{Variedades Analíticas}

Seja $\mathbb{K}$ o corpo dos números reais ou complexos. Indicamos por $\mathcal{O}_{N}$ o conjunto de germes de funções analíticas $\left(\mathbb{K}^{N}, 0\right) \rightarrow \mathbb{K}$. Este conjunto é um anel local (possui um único ideal maximal), cujo ideal maximal é dado por $\mathfrak{m}=\left\{f \in \mathcal{O}_{N} / f(0)=0\right\}$.

Usaremos a notação $\mathbb{C}\left[x_{1}, x_{2}, \ldots, x_{N}\right]$ para representar o anel dos polinômios com variáveis $x_{1}, \ldots, x_{N}$ sobre $\mathbb{C}$.

Definição 1.1. Seja $Y \subset \mathbb{C}^{N}$. Se existe um subconjunto $T \subset \mathbb{C}\left[x_{1}, x_{2}, \ldots, x_{N}\right]$ tal que $Y$ é o conjunto de zeros comuns a todos os polinômios de $T$, dizemos que $Y$ é um conjunto algébrico.

Definição 1.2. Um subconjunto $Y \subset \mathbb{C}^{N}$ é chamado subconjunto analítico complexo de $\mathbb{C}^{N}$ se para cada ponto $y \in \mathbb{C}^{N}$ existe uma vizinhança $U$ de y e funções holomorfas $f_{1}, \ldots, f_{m}$ em $U$ tal que

$$
Y \cap U=\left\{x \in U \mid f_{1}=\cdots=f_{m}=0\right\} .
$$

Ou seja Y localmente é o conjunto de zeros de funções holomorfas.

Definição 1.3. Um conjunto $Y$ é chamado conjunto analítico ou variedade analítica (complexa) se em uma vizinhaça $U^{\prime}$ de cada um do seus pontos, $U^{\prime}$ é um subconjunto analítico. 
Definição 1.4. Sejam $Y_{1}, Y_{2} \subset \mathbb{C}^{N}$ conjuntos analíticos, $Y_{1}$ e $Y_{2}$ são equivalentes em y se existe uma vizinhança $U$ de y tal que $Y_{1} \cap U=Y_{2} \cap U$. As correspondentes classes de equivalências são chamadas germes de conjuntos analíticos em y.

\subsection{Estratificação de Whitney}

Definição 1.5. Seja $X$ uma variedade analítica complexa de dimensão (complexa) d. Uma estratificação de $X$ é dada por uma filtração

$$
\emptyset=X_{-1} \subset X_{0} \subset \cdots \subset X_{d-1} \subset X_{d}=X
$$

tal que cada $V_{i}=X_{i} \backslash X_{i-1}$ é uma variedade complexa suave de dimensão complexa $i$. Chamamos as variedades $V_{i}$ de estratos (não necessariamente conexos) da estratificação.

Definição 1.6. Dizemos que a estratificação $\left\{V_{i}\right\}$ de $X$ satisfaz à condição de fronteira, se para cada par de estratos $\left(V_{1}, V_{2}\right)$ tais que $V_{1} \cap \bar{V}_{2} \neq \emptyset$ então $V_{1} \subset \bar{V}_{2}$.

Definição 1.7. A estratificação $\left\{V_{i}\right\}$ satisfaz as condições de Whitney se para todo par de estratos $(V, W)$ tais que $W \subset \bar{V}$ e para todo $y \in W$ temos:

a) Para toda seqüência $x_{i} \in V, x_{i} \rightarrow y$, e tal que o limite

$$
\lim _{i \rightarrow \infty} T_{x_{i}}(V)=T
$$

existe na grassmaniana correspondente, então $T_{y}(W) \subset T$.

b) Se além disso temos uma seqüência $y_{i} \in W, y_{i} \rightarrow$ y e tal que o limite de direções

$$
\lim _{i \rightarrow \infty} \overline{x_{i} y_{i}}=\lambda
$$

existe no espaço projetivo, então $\lambda \subset T$.

Exemplo 1.8. Consideremos o cone $C$ cujo vértice é a origem, e seja $\{Y, V\}$ uma estratificação onde $Y$ é uma geratriz do cone e $V=C-Y$. Esta estratificação não é de Whitney pois as condições da Definição 1.7 não são satisfeitas, como se mostra na figura 1.1 .

\subsection{Transformação de Nash e o Fibrado de Nash}

Definição 1.9. Seja $X$ uma variedade analítica complexa de dimensão d, mergulhada em uma variedade suave $M$ de dimensão $m, X \subset \Omega \subset M, \Omega$ um aberto em $M$. Sobre $M$ definimos o fibrado $\widehat{M}$, cuja fibra $\widehat{M}_{x}$ sobre $x \in M$ é $G r(d, m)$, conjunto dos d-subespaços em $T_{x}(M) \cong$ 

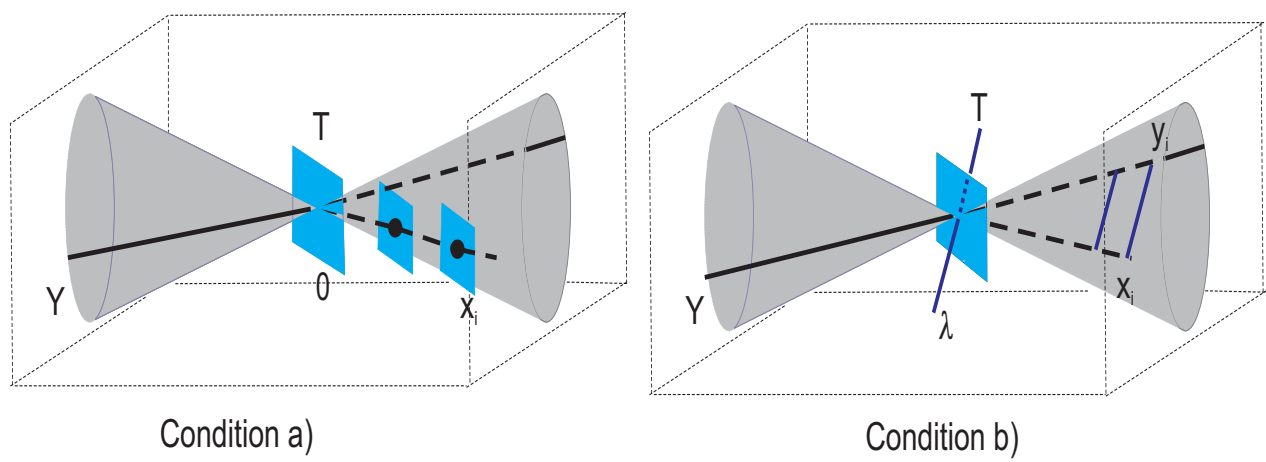

Figura 1.1: Estratificação de Whitney, condições a) e b) não satisfeitas.

$\mathbb{C}^{m}$. Denotamos por $\pi: \widehat{M} \rightarrow M$ a projeção do fibrado. Sobre a parte regular de $X$ temos uma seção dada por:

$$
\begin{array}{rlc}
s: X_{\text {reg }} & \rightarrow & \widehat{M} \\
x & \mapsto \quad\left(x, T_{x} X_{r e g}\right) .
\end{array}
$$

A transformação de Nash $\widehat{X}$ de X é ofecho em $\widehat{M}$ da imagem de s. Quer dizer que:

$$
\widehat{X}=\overline{\left\{(x, W) / x \in X_{\text {reg }}, W=T_{x} X_{\text {reg }}\right\}} \subset X \times G r(d, m) .
$$

Segundo a definição temos o seguinte diagrama:

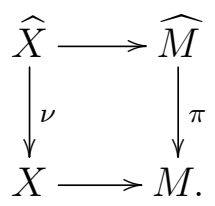

A aplicação $\nu: \widehat{X} \rightarrow X$ é a restrição de $\pi \grave{a} \widehat{X}$.

Se $x \in X$ é um ponto singular então:

$$
\nu^{-1}(x)=\left\{(x, T) / \exists x_{n} \rightarrow x, x_{n} \in X_{r e g}, T=\lim _{x_{n} \rightarrow x}\left(T_{x_{n}} X\right)\right\}
$$

Definição 1.10. Denotemos por E o fibrado tautológico sobre $\widehat{M}$. A fibra $E_{P}$ no ponto $(x, P) \in$ $\widehat{M}$ é o conjunto de vetores $v$ no $d$ - plano $P \in \widehat{M}_{x}$. 


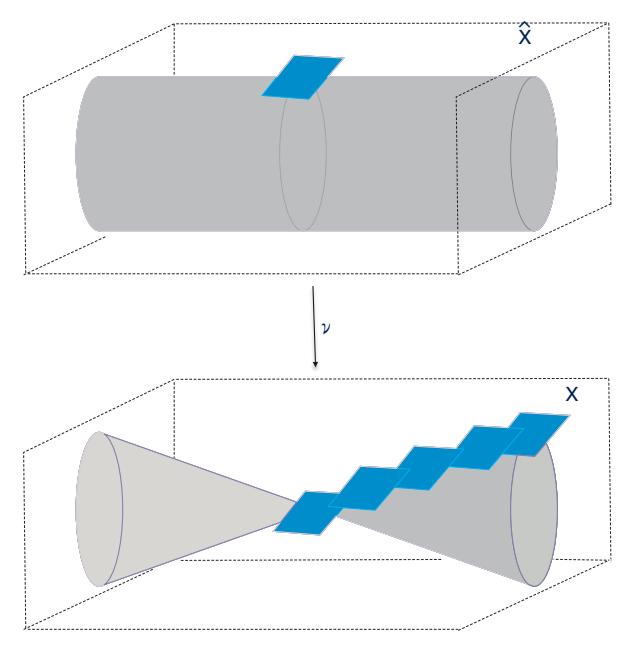

Figura 1.2: Nash

$$
E_{P}=\left\{v(x) \in T_{x} M: v(x) \in P\right\}
$$

Definimos então o fibrado de Nash $\widehat{E}$ sobre $\widehat{X}$, como sendo a restrição do fibrado tautológico sobre $\widehat{X}$, quer dizer, $\widehat{E}=\left.E\right|_{\widehat{X}}$. Então $\left.\widehat{E}\right|_{\widehat{X}_{\text {reg }}}$ pode ser identificado com $T\left(X_{\text {reg }}\right)$ onde $\widehat{X}_{\text {reg }}=$ $\nu^{-1}\left(X_{\text {reg }}\right) \cong X_{\text {reg }} e$

$$
\widehat{E}=E \times_{\widehat{M}} \widehat{X}=\{(v(x), \tilde{x}) \in E \times \widehat{X}: v(x) \in \tilde{x}\}
$$

Um elemento em $\widehat{E}$ é representado por $(x, P, v)$ onde $x \in X, P$ é um $d$-plano em $\nu^{-1}(x) \mathrm{e}$ $v$ é um vetor em $P$. Se $x \in X_{\text {reg }}$, então $P=T_{x}\left(X_{\text {reg }}\right)$.

\subsection{Variedades Polares e Multiplicidade Polar}

Nesta seção introduzimos as variedades polares e as multiplicidades polares. A principal referência a seguir é [39].

Seja $f: X \rightarrow S$ um morfismo de espaços analíticos reduzidos tais que as fibras de $f$ são suaves e de dimensão $d=\operatorname{dim} X-\operatorname{dim} S$ sobre o complementar de um conjunto fechado $F$. De maneira geral, podemos mergulhar $X \subset S \times \mathbb{C}^{N}$ como o diagrama.

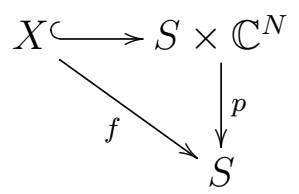


Proposição 1.11. Seja $N$ um inteiro e seja:

$$
(0) \subset D_{N-1} \subset D_{N-2} \subset \cdots \subset D_{1} \subset D_{0}=\mathbb{C}^{N}
$$

uma sequência de subespaços vetoriais em $\mathbb{C}^{N}$, tal que codim $D_{i}=i$. Para cada inteiro $0 \leqslant k \leqslant d$, chamamos $k$-ésima variedade de Schubert projetiva associada à $\mathcal{D}$, denotada por $c_{k}(\mathcal{D})$, ao subespaço da Grassmanniana $G$ de d-planos de $\mathbb{C}^{N}$, definido por:

$$
c_{k}(\mathcal{D})=\left\{T \in G / \operatorname{dim}\left(T \cap D_{d-k+1}\right) \geqslant k\right\} .
$$

Para todo $\mathcal{D}$, o conjunto $c_{k}(\mathcal{D})$ é munido naturalmente de uma estrutura de subvariedade algébrica reduzida de $G$, de codimensão $k$ em $G$.

Denotemos por $\mathcal{W}_{k}$ o conjunto dos sub-espaços genéricos, $D_{d-k+1}$ em $\mathbb{C}^{N}$, ver [39, Pag. 419] para as propriedades de generalidade. $\mathrm{O}$ conjunto $\mathcal{W}_{k}$ é aberto e denso nas Grassmanianas de sub-espaços de codimensão $d-k+1 \mathrm{em} \mathbb{C}^{N}$.

Uma projeção $p: \mathbb{C}^{N} \rightarrow \mathbb{C}^{d-k+1}$ é uma projeção linear genérica se ker $p=D_{d-k+1} \in \mathcal{W}_{k}$.

Teorema 1.12. [39 V 1.3.2] Suponha que $f: X \rightarrow S$ seja um morfismo liso, isto é, $f$ é plano e as fibras são suaves de codimensão $d=\operatorname{dim} X-\operatorname{dim} S$, fora de um conjunto fechado F. Seja $p: \mathbb{C}^{N} \rightarrow \mathbb{C}^{d-k+1}$ uma projeção linear genérica. Para cada $x \in X-F$, a fibra $X(f(x))=f^{-1}(f(x))$ de $f$ em $X$ é não singular em $x$, contida em $\{f(x)\} \times \mathbb{C}^{N}$. Notemos por $\pi_{x}: X(f(x)) \rightarrow \mathbb{C}^{d-k+1}$ a restrição à $X(f(x))$ da projeção $p$.

Seja $P_{k}^{0}(X, f, p)=\left\{x \in X-F \mid x \in \Sigma \pi_{x}\right\}$, onde $\Sigma \pi_{x}$ é o conjunto de pontos críticos de $\pi_{x}$.

O fecho $P_{k}(X, f, p)$ de $P_{k}^{0}(X, f, p)$ em $X$, é um subespaço analítico fechado de $X$, de codimensão $k$ em $X$ ou vazio.

Então temos a seguinte definição:

Definição 1.13. Seja $f:(X, 0) \rightarrow(S, 0)$ um morfismo como acima, tal que $(X, 0) \subset(S, 0) \times$ $\left(\mathbb{C}^{N}, 0\right)$, e seja $D_{d-k+1} \subset \mathbb{C}^{N}$ de codimensão $d-k+1$ contido em $\mathcal{W}_{k}$. Chamamos variedade polar local relativa de codimensão $k$ associada a $f$ e a p ao subespaço analítico

$$
P_{k}(X, f, p)=\overline{P_{k}^{0}(X, f, p)}
$$

onde $p: \mathbb{C}^{N} \rightarrow \mathbb{C}^{d-k+1}$ é uma aplicação linear tal que $D_{d-k+1}=\operatorname{ker} p$.

Observação 1.14. Podemos ver que

1. Se $S$ é um ponto e f é a aplicação constante, então denotamos a variedade polar por $P_{k}(X, p)$ e a chamaremos variedade polar absoluta. 


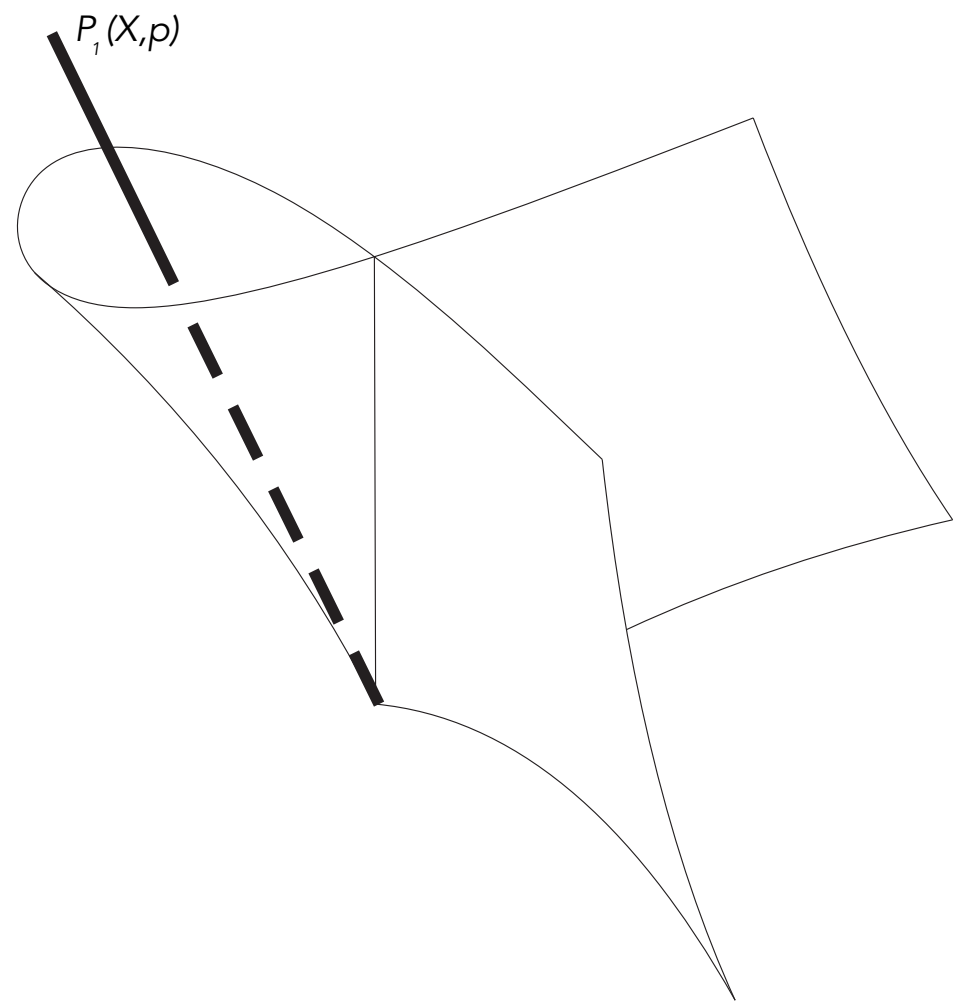

Figura 1.3: $P_{1}(X, p)$

Exemplo 1.15. Calculemos as variedades polares da seguinte variedade singular:

$$
X=\left\{(x, y, t) \in \mathbb{C}^{3}: y^{2}-x^{3}-t x^{2}=0\right\}
$$

1. $P_{0}(X, p)=X, p: \mathbb{C}^{3} \rightarrow C^{3}, p(x, y, t)=(x, y, t)$.

2. $P_{1}(X, p)=\left\{(x, y, t) \in \mathbb{C}^{3}: x+t=0, y=0\right\}, p: \mathbb{C}^{3} \rightarrow \mathbb{C}^{2}, p(x, y, t)=(x, t)$.

Denotamos por $m_{k}(X, f)=m_{0}\left(P_{k}(X, f, p)\right)\left(\operatorname{com} D=\operatorname{ker} p \in \mathcal{W}_{k}\right)$ a $k$-ésima multiplicidade polar da variedade polar relativa $P_{k}(X, f, p)$. Se $f$ é uma aplicação constante, denotamos a multiplicidade polar absoluta por $m_{k}(X)$.

Teorema 1.16. Seja $f:(X, 0) \rightarrow(S, 0)$ um morfismo de espaços analíticos. Para todo $S$ mergulho local $(X, 0) \subset(S, 0) \times\left(\mathbb{C}^{N}, 0\right)$, toda escolha de coordenadas, e para todo inteiro $k, 0 \leqslant k \leqslant d=\operatorname{dim} X-\operatorname{dim} S$, existe um aberto de Zariski denso $V_{k} \subset W_{k}$, tal que as multiplicidades $m_{k}(X, f)$ independem do $D_{d-k+1} \in V_{k}$.

No Capítulo 3, definiremos $m_{d}(X)$ para variedades de dimensão $d$ que admitem perturbações suaves (suavização). 


\subsection{Fecho Integral de Ideais e de Módulos}

A principal referência para esta seção na qual daremos algumas noções de fecho integral é [21].

Definição 1.17. Seja I um ideal no anel $A$ e $h \in A$, dizemos que h está no fecho integral de $I$, denotado por $\bar{I}$, se existe um polinômio mônico $P(z)=z^{n}+\sum_{i=0}^{n-1} a_{i} z^{i}, a_{i} \in I^{n-i}$ tal que $P(h)=0$

Considere $(X, x)$ uma variedade analítica complexa equidimensional, definida por $F$ : $\left(\mathbb{C}^{N}, 0\right) \rightarrow\left(\mathbb{C}^{p}, 0\right)$ e $A=\mathcal{O}_{X, x}$ seu anel local. Neste caso $\bar{I}$ tem propriedades interessantes.

Dada $\phi:(\mathbb{C}, 0) \rightarrow(X, x)$ uma aplicação, podemos associar uma aplicação induzida $\phi^{*}$ : $\mathcal{O}_{X, x}^{p} \rightarrow \mathcal{O}_{\mathbb{C}, 0}$ definida por $\phi^{*}(h)=h \circ \phi$.

Proposição 1.18. [21] Seja $I \subset \mathcal{O}_{X, x}$ um ideal, $X$ um conjunto analítico complexo. Então as seguintes condições são equivalentes.

1. $h \in \bar{I}$.

2. Para cada escolha de geradores $\left(g_{i}\right)$ de $I$, existe uma vizinhança $U$ de $x$ e uma constante $C>0$ tal que:

$$
\|h(z)\| \leqslant C \sup _{i}\left\|g_{i}(z)\right\|, \text { para todo } z \in U
$$

3. Para cada $\phi:(\mathbb{C}, 0) \rightarrow(X, x), h \circ \phi \in\left(\phi^{*}(I)\right) \mathcal{O}_{1}$.

4. Existe $L, \mathcal{O}_{X, x}$ módulo faithful de tipo finito tal que h.I $\subset$ I.L. L é um $\mathcal{O}_{X, x}$ módulo faithful se $\operatorname{Ann}_{\mathcal{O}_{X, x}}(L)=\left\{r \in \mathcal{O}_{X, x} \mid \forall l \in L, r l=0\right\}=0$,

A seguir daremos a definição de fecho integral de módulos, e algumas das suas propriedades.

Definição 1.19. Seja $(X, x)$ um germe analítico complexo, $M \subset \mathcal{O}_{X, x}^{p}$ um submódulo. Então $h \in \bar{M}$ se para todo $\phi:(\mathbb{C}, 0) \rightarrow(X, x), h \circ \phi \in\left(\phi^{*}(M)\right) \mathcal{O}_{1}$.

Exemplo 1.20. Seja $X=\mathbb{C}^{2}, M=\langle(x, 0),(0, y),(y, x)\rangle \subset \mathcal{O}_{2}^{2}$. Então $\bar{M}=m_{2} \mathcal{O}_{2}^{2}$. Seja $\phi(t)=\left(\phi_{1}(t), \phi_{2}(t)\right)$, então $\left(\phi^{*} M\right) \mathcal{O}_{1}=\left\langle\left(t^{n}, 0\right),\left(0, t^{n}\right)\right\rangle$, onde $n=\min _{i=1,2}\left\{o\left(\phi_{i}\right)\right\}$ e o $\left(\phi_{i}\right)$ denota a ordem de $\phi_{i}$.

Denotemos por $J_{k}(M)$ o $k$-ésimo ideal de Fitting de $M$, i.e. o ideal gerado pelos zeros dos menores $k \times k$, da matriz A, dado pelos geradores de $M$. O módulo gerado por $M$ e $h \in \mathcal{O}_{X, x}^{p}$ será denotado por $(h, M)$.

Proposição 1.21. Se $M \subset \mathcal{O}_{X, x}^{p}$ é um submódulo, $h \in \mathcal{O}_{X, x}^{p}$ e rank $(h, M)=k$ sobre cada componente de $(X, x)$. Então $h \in \bar{M}$ se somente se, $J_{k}(h, M) \subset \overline{J_{k}(M)}$. 
Seja $F: \mathbb{C}^{N} \rightarrow \mathbb{C}^{p}$, uma função analítica complexa, denotemos por $J M(F) \subset \mathcal{O}_{X, x}^{p}$ o submódulo jacobiano de $F$ gerado por $\left\langle\frac{\partial F}{\partial x_{1}}, \ldots, \frac{\partial F}{\partial x_{N}}\right\rangle$. Os módulos de nosso interesse são os módulos jacobianos, $J M(F)$, e as reduções destes $(P \subset M$ é redução de $M$ se $\bar{M}=\bar{P}$.)

Corolário 1.22. [21] Seja $(X, 0)$ uma d-variedade analítica complexa equidimensional definida por $F: \mathbb{C}^{N} \rightarrow \mathbb{C}^{p}$. Então $P$ é redução de $J M(F)$ se, somente $\forall \phi:(\mathbb{C}, 0) \rightarrow(X, 0)$ tal que $\phi(\mathbb{C}-\{0\}) \subset X-\Sigma X$, temos que $\left(\phi^{*} P\right) \mathcal{O}_{1}=\left(\phi^{*} M\right) \mathcal{O}_{1}$.

Proposição 1.23. Seja $(X, x)$ uma variedade analítica complexa equidimensional, definida por $F:\left(\mathbb{C}^{N}, 0\right) \rightarrow\left(\mathbb{C}^{p}, 0\right)$ e seja $P$ um submódulo de $J M(F)$ tal que $(h, P)=J M(F)$. Temos que $h \in \bar{P}$ se, somente se, para toda curva $\phi:(\mathbb{C}, \mathbb{C}-0,0) \rightarrow(X, X-\Sigma X, 0)$ e $\psi: \mathbb{C} \rightarrow$ $\operatorname{Hom}\left(\mathbb{C}^{p}, 0\right)$, temos $\psi\left(\phi^{*}(h)\right) \in I_{\psi}\left(\phi^{*}(P)\right)$, onde $I_{\psi}\left(\phi^{*}(P)\right)$ é o ideal de $\mathcal{O}_{1}$ gerado aplicando $\psi$ aos geradores de $\phi^{*}(N)$.

\subsection{Número de Milnor de uma Curva em $\mathbb{C}^{N}$}

Nesta parte definimos o número de Milnor para curvas espaciais seguindo os artigos [7, 32, 6].

Seja $(X, 0) \subset\left(\mathbb{C}^{N}, 0\right)$ uma curva singular reduzida. Seja $\Omega_{(X, 0)}^{1}$ o módulo de 1-formas holomorfas sobre $X$ (módulo das diferenciais de Kähler) e $\omega_{(X, 0)}$ o módulo dualizante de Grothendieck

$$
\omega_{(X, 0)}=\operatorname{Ext}_{\mathcal{O}_{\left(\mathbb{C}^{N}, 0\right)}^{N-1}}\left(\mathcal{O}_{X, 0}, \Omega_{\left(\mathbb{C}^{N}, 0\right)}^{N}\right)
$$

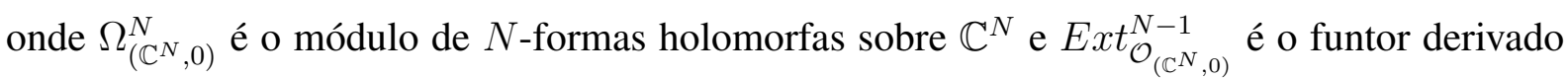
Ext. Seja $\eta:\left(\overline{X, x_{0}}\right) \rightarrow(X, 0)$ a normalização, onde $\left(\overline{X, x_{0}}\right)$ é o multigerme $\left(\bar{X}, \eta^{-1}(0)\right)$. Como $\Omega^{1}$ é um funtor contravariante e $\omega$ é um funtor covariante, podemos usar a funtorialidade para obter a aplicação $c_{X}$.

$$
c_{X}: \Omega_{(X, 0)}^{1} \rightarrow \Omega_{\left(\overline{X, x_{0}}\right)}^{1} \cong \omega_{\left(\overline{X, x_{0}}\right)} \rightarrow \omega_{(X, 0)} .
$$

Também temos a aplicação derivada " $d$ ", compondo com $c_{X}$ obtemos uma nova aplicação ao qual chamamos de " $d$ "

$$
d: \mathcal{O}_{(X, 0)} \stackrel{d}{\longrightarrow} \Omega_{(X, 0)}^{1} \stackrel{c_{X}}{\longrightarrow} \omega_{(X, 0)} .
$$

Definição 1.24. [7] Seja $(X, 0)$ uma curva singular reduzida. Defininimos o número de Milnor, $\mu$, como a dimensão da seguinte álgebra:

$$
\mu=\mu(X, 0)=\operatorname{dim}_{\mathbb{C}} \frac{\omega_{(X, 0)}}{d\left(\mathcal{O}_{X, 0}\right)}
$$


Daremos uma definição equivalente para $\omega_{(X, 0)}$, usando as formas diferenciais de Rosenlicht. Seja $\bar{x}_{0}=\eta^{-1}(0)$ e denotemos por $\Omega_{\left(\overline{X, x_{0}}\right)}^{1}\left(x_{0}\right)$ o multigerme de formas meromorfas sobre $\left(\overline{X, x_{0}}\right)$ com no máximo um polo (de qualquer ordem) em $\bar{x}_{0}$. Definimos o conjunto das formas diferenciais regulares de Rosenlicht como sendo:

$$
\omega_{(X, 0)}^{R}=\eta_{*}\left\{\alpha \in \Omega_{\left(\overline{X, x_{0}}\right)}^{1}\left(x_{0}\right) / \sum_{p \in \bar{x}_{0}} r e s_{p}(f \alpha)=0, \forall f \in \mathcal{O}_{(X, 0)}\right\}
$$

Outra vez obtemos a aplicação $d$,

$$
d: \mathcal{O}_{(X, 0)} \stackrel{d}{\longrightarrow} \Omega_{(X, 0)}^{1} \stackrel{c_{X}}{\longrightarrow} \omega_{(X, 0)}
$$

Assim

$$
\mu(X)=\operatorname{dim}_{\mathbb{C}} \frac{\omega_{(X, 0)}^{R}}{d \mathcal{O}_{(X, 0)}} .
$$

A definição de Grothendieck tem a vantagem de ser funtorial e pode ser estendida a várias dimensões. Mas pelo contrário a definição de Rosenlicht é mais usada para os cálculos.

Em [7] é dada otra definição de número de Milnor que coincide com o número de Milnor para interseções completas.

Seja $\eta:\left(\overline{X, x_{0}}\right) \rightarrow(X, 0)$ a normalização, denotemos por $\delta=\operatorname{dim}_{\mathbb{C}} \frac{\eta_{*} \mathcal{O}_{\overline{\left(X, x_{0}\right)}}}{\mathcal{O}_{X, 0}}$ e por $r$ o número de ramos da curva $(X, 0$

Observação 1.25. [7] O número de Milnor da curva $(X, 0)$ satisfaz a fórmula de Milnor

$$
\mu(X)=2 \delta-r+1
$$

No seguinte exemplo calculamos o número de Milnor de $C$ usando a definição equivalente à definição 1.24 .

Exemplo 1.26. Seja $C=V\left(z\left(z+x^{2}\right), y\left(z+x^{2}\right), y^{2}-x z\right)$, curva singular. Vamos calcular o número de Milnor da curva C. A curva tem dois ramos, o primeiro ramo é dado pela seguinte normalização

$$
\begin{aligned}
& \eta_{1}: \mathbb{C} \rightarrow \quad C \\
& t \mapsto\left(t^{2},-t^{3},-t^{4}\right)
\end{aligned}
$$

O segundo ramo é parametrizado por:

$$
\begin{aligned}
\eta_{2}: \mathbb{C} & \rightarrow C \\
s & \mapsto(s, 0,0)
\end{aligned}
$$


Assim, temos a aplicação induzida por $\eta$,

$$
\eta_{*}: \mathbb{C}\left\{\left(\begin{array}{c}
t^{2} \\
s
\end{array}\right),\left(\begin{array}{c}
-t^{3} \\
0
\end{array}\right),\left(\begin{array}{c}
-t^{4} \\
0
\end{array}\right)\right\} \rightarrow \mathbb{C}\{t\} \oplus \mathbb{C}\{s\}
$$

Vamos calcular uma base para as 1-formas diferenciais de Rosenlicht. Um elemento $\alpha \in$ $\Omega_{\left(\overline{X, x_{0}}\right)}^{1}\left(x_{0}\right)$ pode ser expresso em séries da seguinte forma:

$$
\left(\begin{array}{c}
\left(\sum_{m=1}^{r} a_{m} t^{-m}+\sum_{m=0}^{\infty} b_{m} t^{m}\right) d t \\
\left(\sum_{k=1}^{l} c_{k} s^{-k}+\sum_{k=0}^{\infty} d_{k} s^{k}\right) d s
\end{array}\right)
$$

Temos que $\left(\begin{array}{l}1 \\ 1\end{array}\right) \in \mathcal{O}_{(X, 0)}$, então $a_{1}+c_{1}=0$

$$
\begin{aligned}
& \left(\begin{array}{c}
t^{2} \\
s
\end{array}\right) \in \mathcal{O}_{(X, 0)} \text {, então } a_{3}+c_{2}=0 \\
& \left(\begin{array}{c}
-t^{3} \\
0
\end{array}\right) \in \mathcal{O}_{(X, 0)} \text {, então } a_{4}=0 \text {, assim } a_{m}=0, \forall m \geqslant 4 \text { e } c_{k}=0, \forall m \geqslant 2 .
\end{aligned}
$$

Logo temos que

$$
\begin{gathered}
\left(\begin{array}{c}
\left(a_{3} t^{-3}+a_{2} t^{-2}+a_{1} t^{-1}+\sum_{m=0}^{\infty} b_{m} t^{m}\right) d t \\
\left(-a_{3} s^{-2}+-a_{1} s^{-1}+\sum_{k=0}^{\infty} d_{k} s^{k}\right) d s
\end{array}\right) \in \omega_{X}^{R} \\
\omega_{X}^{R}=\left(\begin{array}{c}
t^{-3} d t \\
-s^{-2} d s
\end{array}\right) \mathbb{C} \oplus\left(\begin{array}{c}
t^{-2} d t \\
0
\end{array}\right) \mathbb{C} \oplus\left(\begin{array}{c}
t^{-1} d t \\
-s^{-1} d s
\end{array}\right) \mathbb{C} \oplus\left(\begin{array}{c}
1 d t \\
0
\end{array}\right) \mathbb{C}\{t\} \oplus\left(\begin{array}{c}
0 \\
1 d s
\end{array}\right) \mathbb{C}\{s\}
\end{gathered}
$$

Para calcular $\mu$, precisamos descrever $d \mathcal{O}_{X}$ :

$$
d \mathcal{O}_{X, 0}=\left(\begin{array}{c}
2 t \\
1
\end{array}\right) \mathbb{C} \oplus\left(\begin{array}{c}
t^{2} \\
0
\end{array}\right) \mathbb{C}\{t\} \oplus\left(\begin{array}{l}
0 \\
s
\end{array}\right) \mathbb{C}\{s\}
$$

Assim, temos que o quociente é gerado por

$$
\frac{\omega_{(X, 0)}^{R}}{d \mathcal{O}_{(X, 0)}}=\left(\begin{array}{c}
t^{-3} d t \\
-s^{-2} d s
\end{array}\right) \mathbb{C} \oplus\left(\begin{array}{c}
t^{-2} \\
0
\end{array}\right) \mathbb{C} \oplus\left(\begin{array}{c}
t^{-1} \\
-s^{-1}
\end{array}\right) \mathbb{C} \oplus\left(\begin{array}{c}
1 d t \\
0
\end{array}\right) \mathbb{C} \oplus\left(\begin{array}{c}
t d t \\
0
\end{array}\right) \mathbb{C}
$$

Portanto $\mu=5$. 


\section{Variedades Determinantais}

Neste capítulo apresentaremos as definições básicas sobre variedades determinantais, com base no artigo de W. Èbeling e S. M. Guseľn-Zade [23]. O objetivo é definir as variedades essencialmente isoladas e estudar suas propriedades. Estas singularidades generalizam as singularidades isoladas. Mostramos também que elas podem ser estudadas através da teoria de singularidades de matrizes. As referências para este estudo são as teses de Frühbis-Krüger [17] e Silva Pereira [34]

\subsection{Variedades Determinantais Essencialmente Isoladas}

Denotemos por $M_{m, n}$ o conjunto das matrizes $m \times n$ com entradas complexas.

Definição 2.1. Para cada $t, 1 \leqslant t \leqslant \min \{m, n\}$, seja $M_{m, n}^{t}$ o subconjunto de $M_{m, n}$ cujos elementos são matrizes de posto menor do que $t$ :

$$
M_{m, n}^{t}=\left\{A \in M_{m, n} / \operatorname{rank}(A)<t\right\} .
$$

Este conjunto é uma variedade singular de codimensão $(m-t+1)(n-t+1)$ em $M_{m, n}$, chamada variedade determinantal genérica.

O conjunto singular de $M_{m, n}^{t}$ é $M_{m, n}^{t-1}$. Temos que $M_{m, n}^{t}=\cup_{i=1, \ldots, t}\left(M_{m, n}^{i} \backslash M_{m, n}^{i-1}\right) \mathrm{e}$ $\left\{M_{m, n}^{i} \backslash M_{m, n}^{i-1}\right\}_{i=1, \cdots, t}$ é uma estratificação de Whitney de $M_{m, n}^{t}$, ver [1].

Seja $F: \mathbb{C}^{N} \rightarrow M_{m, n}$ uma aplicação dada pela matriz $m \times n, F(x)=\left(f_{i j}(x)\right)$, cujas entradas são funções analíticas complexas definidas sobre um domínio aberto $U \subset \mathbb{C}^{N}$.

Definição 2.2. A variedade determinantal de tipo $(m, n, t)$ é a pré-imagem $F^{-1}\left(M_{m, n}^{t}\right)$ da variedade $M_{m, n}^{t}$ com a condição que a codimensão seja $(n-t+1)(m-t+1)$. 
A imagem de uma aplicação genérica $F$ pode interceptar a variedade $M_{m, n}^{i} \operatorname{com} i<t$ (além disso a correspondente variedade determinantal $X=F^{-1}\left(M_{m, n}^{t}\right)$ tem singularidades). Uma aplicação genérica $F$ intercepta o estrato $M_{m, n}^{i} \backslash M_{m, n}^{i-1}$ da variedade $M_{m, n}^{t}$ transversalmente. Quer dizer, nos pontos correspondentes, a variedade determinantal tem um tipo de singularidades que depende apenas de $i=\operatorname{rank} F(x)+1$.

Definição 2.3. Um ponto $x \in X=F^{-1}\left(M_{m, n}^{t}\right)$ é chamado essencialmente não singular se, neste ponto, a aplicação $F$ é transversal ao estrato correspondente da variedade $M_{m, n}^{t}$ (isto é, ao estrato $\left\{M_{m, n}^{i} \backslash M_{m, n}^{i-1}\right\}$, com $\left.i=\operatorname{rank} F(x)+1\right)$.

Sejam $I \subset\{1, \cdots, m\}$ e $J \subset\{1, \cdots, n\}$ tais que $|I|=|J|=i$. Denotemos por $m_{I J}(x)$ o menor $i \times i$ correspondente da matriz $F(x)$ e por $\left\{d m_{I J}^{i}(x)\right\}$ o conjunto de diferenciais de todos os menores $m_{I J}(x)$.

Em um ponto essencialmente não singular $x_{0} \in X \operatorname{com} \operatorname{rank} F\left(x_{0}\right)=i-1$, temos que o posto da matriz $\left\{d m_{I J}^{i}\right\}$ é $(m-i+1)(n-i+1)$.

Para $1 \leqslant i \leqslant t$, denotemos por $X_{i}=F^{-1}\left(M_{m, n}^{i}\right)$. Em uma vizinhança do ponto essencial não singular $x_{0}$, temos que a subvariedade $X_{i} \backslash X_{i-1}=\{x \in X / \operatorname{rank} F(x)=i-1\}$ é não singular de dimensão $N-(m-i+1)(n-i+1)$.

Em um ponto essencialmente singular $x \in X$ temos,

$$
\operatorname{rank}\left(\left\{d m_{I J}^{i}\right\}\right)_{I J}<(m-i+1)(n-i+1) .
$$

Definição 2.4. Um germe $(X, 0) \subset\left(\mathbb{C}^{N}, 0\right)$ de variedade determinantal de tipo $(m, n, t)$ tem um ponto essencialmente singular isolado na origem (EIDS), se existe uma vizinhança $U$ da origem em $\mathbb{C}^{N}$ tal que todos os pontos singulares de $X$ em $U-\{0\}$ são pontos essencialmente não singulares.

Quando $t=1$ ou $N \leqslant(m-t+2)(n-t+2)$ a singularidade da variedade determinantal é isolada e satisfaz a seguinte definição.

Definição 2.5. Seja $X$ uma variedade determinantal de tipo $(m, n, t)$, definida por $F$ e satisfazendo: $t=1$ ou $N \leqslant(m-t+2)(n-t+2)$. Dizemos que $(X, 0)$ é uma variedade determinantal com singularidade isolada em 0 ou IDS se $X$ é suave em $x$ e rank $F(x)=t-1$, para todo $x \neq 0$ em uma vizinhança da origem.

De agora em diante vamos supor que a variedade determinantal $X$ é definida por uma aplicação $F:\left(\mathbb{C}^{N}, 0\right) \rightarrow M_{m, n} \operatorname{com} F(0)=0$. Isto não é uma restrição porque se $F(0) \neq 0$ e $\operatorname{rankF(0)}=s>0$, então $X$ é uma variedade determinantal de tipo $(m-s, n-s, t-s)$ definida pela aplicação $F^{\prime}:\left(\mathbb{C}^{N}, 0\right) \rightarrow M_{m-s, n-s} \operatorname{com} F^{\prime}(0)=0$. Vamos supor também que a dimensão de $X$ é maior do que 0 , ou seja $N>(m-t+1)(n-t+1)$. 
Exemplo 2.6. Considere $X=F^{-1}\left(M_{2,3}^{2}\right)$, onde $F$ é definida por

$$
\begin{aligned}
F: \mathbb{C}^{4} & \rightarrow \\
(x, y, z, w) & \mapsto\left(\begin{array}{ccc}
M_{2,3} \\
z & y & x \\
w & z & y
\end{array}\right)
\end{aligned}
$$

$A$ variedade $X$ é definida pelos zeros de $z^{2}-w y, y^{2}-z x, z y-w x$ e tem singularidade isolada na origem.

Definição 2.7. Consideramos uma perturbação de F no seguinte sentido,

$$
\begin{aligned}
\widetilde{F}: \mathbb{C}^{N} \times \mathbb{C} & \rightarrow M_{m, n} \\
(x, t) & \mapsto \widetilde{F}(x, s)
\end{aligned}
$$

onde $\widetilde{F}(x, 0)=F(x)$ e $\widetilde{F}_{s}: \mathbb{C}^{N} \rightarrow M_{m, n}$ é transversal a todos os estratos $M_{m, n}^{i} \backslash M_{m, n}^{i-1}$, com $i \leqslant t$, para todo $s \neq 0$ suficiente próximo de zero. Então $\widetilde{X}_{s}=\widetilde{F}_{s}^{-1}\left(M_{m, n}^{t}\right), s \neq 0$ é chamada uma suavização essencial de $X$.

Quando $\widetilde{X}$ é suave, dizemos que $\widetilde{X}$ é uma suavização de $X$.

Uma perturbação genérica $\widetilde{F}$ da aplicação $F$ define uma suavização essencial da EIDS $(X, 0)$. Uma suavização essencial em geral não é suave (para $N \geq(m-t+2)(n-t+2))$. $\mathrm{O}$ conjunto singular de $\widetilde{X}_{s}$ é $\widetilde{F}_{s}^{-1}\left(M_{m, n}^{t-1}\right)$. Como $F$ é transversal aos estratos de $M_{m, n}^{t}$, segue que $\widetilde{X}_{s}=\cup_{1 \leq i \leq t} \widetilde{F}_{s}^{-1}\left(M_{m, n}^{i} \backslash M_{m, n}^{i-1}\right)$ é uma estratificação de Whitney de $\widetilde{X}_{s}$.

Uma suavização essencial de uma $\operatorname{EIDS}(X, 0)$ de tipo $(m, n, t)$ é uma suavização, isto é, $\widetilde{X}$ é suave, se $N<(m-t+2)(n-t+2)$.

Teorema 2.8. [40] Seja $(X, 0)$ uma variedade determinantal com singularidade isolada na origem, definida pelos menores $t \times t$ de uma matriz $F_{i j} m \times n$, com entradas em $\mathcal{O}_{N}, 2 \leqslant t \leqslant$ $m \leqslant n$. Se $\operatorname{dim}(X)<m+n-2 t+3$, então $X$ admite uma suavização.

Exemplo 2.9. Seja $X=F^{-1}\left(M_{2,3}^{2}\right)$ a superfície determinantal definida pela função,

$$
\begin{aligned}
F: \mathbb{C}^{4} & \rightarrow \\
(x, y, z, w) & \mapsto\left(\begin{array}{ccc}
z & y+w & x \\
w & x & y
\end{array}\right)
\end{aligned}
$$

Uma suavização essencial, $\widetilde{X}_{s}$, de $X$ é representada pela seguinte matriz:

$$
\begin{aligned}
\widetilde{F}_{s}: \quad U \subset \mathbb{C}^{4} & \rightarrow M_{2,3} \\
(x, y, z, w) & \mapsto\left(\begin{array}{ccc}
z & y+w & x+s \\
w & x & y
\end{array}\right), s \neq 0,
\end{aligned}
$$


então $\widetilde{X}_{s}=\widetilde{F}_{s}^{-1}\left(M_{2,3}^{2}\right)$ é uma suavização de $X$.

Exemplo 2.10. Seja $X=F^{-1}\left(M_{2,3}^{2}\right)$ a 4-variedade determinantal definida pela seguinte matriz

$$
\begin{aligned}
& F: \quad \mathbb{C}^{6} \quad \rightarrow \quad M_{2,3} \\
& (x, y, z, w, v, u) \mapsto\left(\begin{array}{ccc}
x & y & v \\
z & w & x+u^{2}
\end{array}\right)
\end{aligned}
$$

Uma suavização essencial, $\widetilde{X}_{s}$, de $X$ é representada pela seguinte matriz:

$$
\begin{aligned}
\widetilde{F}_{s}: \quad U \subset \mathbb{C}^{6} & \rightarrow M_{2,3} \\
(x, y, z, w, v, u) & \mapsto\left(\begin{array}{ccc}
x+s & y & v \\
z & w & x+u^{2}
\end{array}\right), s \neq 0,
\end{aligned}
$$

então $\widetilde{X}_{s}=\widetilde{F}_{s}^{-1}\left(M_{2,3}^{2}\right)$ é uma suavização essencial de $X$, que tem singularidade em $y=z=$ $w=v=0, x=-s, u= \pm \sqrt{s}$.

Exemplo 2.11. Seja $X=F^{-1}\left(M_{2,4}^{2}\right) \subset \mathbb{C}^{5}$ a superfície determinantal definida por

$$
\begin{aligned}
& F: \quad \mathbb{C}^{5} \quad \rightarrow \quad M_{2,4} \\
& (x, y, z, w, v) \mapsto\left(\begin{array}{cccc}
x & y & z & w \\
y & z & w & v
\end{array}\right)
\end{aligned}
$$

Uma suavização essencial, $\widetilde{X}_{s}$, de X é representada pela matriz:

$$
\begin{aligned}
\widetilde{F}_{s}: \quad U \subset \mathbb{C}^{5} & \rightarrow M_{2,4} \\
(x, y, z, w, v) & \mapsto\left(\begin{array}{cccc}
x & y+s & z & w \\
y & z & w & v
\end{array}\right), s \neq 0,
\end{aligned}
$$

então $\widetilde{X}_{s}=\widetilde{F}_{s}^{-1}\left(M_{2,4}^{2}\right)$ é uma suavização essencial de $X$, que é singular.

\subsection{Singularidades de Matrizes}

Vamos definir os grupos $\mathcal{G}(n, m), \mathcal{K}_{V}$ e $\mathcal{K}_{H}$ que são subgrupos geométricos do grupo de contato $\mathcal{K}$, que agem no espaço das matrizes $m \times n$ com entradas em $\mathcal{O}_{N}$. O grupo $\mathcal{G}(n, m)$ age sobre o espaço das matrizes através de operações de linhas e colunas de matrizes e de mudanças de coordenadas na fonte. A teoria de singularidades pode ser então utilizada, e as 
caracterizações de estabilidade e determinação finita se verificam para estas ações. Poderemos então dar novas caracterizações para as EIDS, tendo como base a teoria de singularidades de matrizes. As principais referências a seguir são [18, 35, 12].

\subsection{1 $\mathcal{K}$ - Equivalência}

Vamos descrever o grupo de contato $\mathcal{K}$ que age sobre o espaço de de germes analíticos $F:\left(\mathbb{C}^{N}, 0\right) \rightarrow\left(\mathbb{C}^{p}, 0\right)$.

$\mathcal{K}=\left\{\Phi:\left(\mathbb{C}^{N+p}, 0\right) \rightarrow\left(\mathbb{C}^{N+p}, 0\right) \mid \Phi\right.$ é um difeomorfismo, $\Phi(x, y)=(\phi(x), \theta(x, y)), \theta(x, 0)=$ 0 , onde $\phi:\left(\mathbb{C}^{N}, 0\right) \rightarrow\left(\mathbb{C}^{N}, 0\right) \rightarrow\left(\mathbb{C}^{N}, 0\right)$ é um difeomorfismo $\}$.

Ou seja, $\mathcal{K}$ consiste de difeomorfismos analíticos $\Phi: \mathbb{C}^{N} \times \mathbb{C}^{p} \rightarrow \mathbb{C}^{N} \times \mathbb{C}^{p}$ tais que existe um difeomorfismo $\phi:\left(\mathbb{C}^{N}, 0\right) \rightarrow\left(\mathbb{C}^{N}, 0\right)$, na qual temos o seguinte diagrama comutativo:

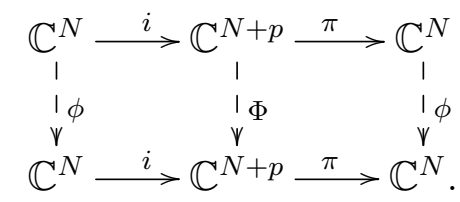

$i: \mathbb{C}^{N} \rightarrow \mathbb{C}^{N+p}$ é a inclusão $i(x)=(x, 0)$ e $\pi: \mathbb{C}^{N+p} \rightarrow \mathbb{C}^{N}$ é a projeção sobre o primeiro fator.

Um elemento $\Phi \in \mathcal{K}$ age sobre $F:\left(\mathbb{C}^{N}, 0\right) \rightarrow\left(\mathbb{C}^{p}, 0\right)$ se $\operatorname{graf}(\Phi . F)=\Phi($ graf $F)$, onde graf $F$ é o gráfico da função $F$.

\subsubsection{A $\mathcal{G}(n, m)$ - Equivalência}

Seja como antes $F:\left(\mathbb{C}^{N}, 0\right) \rightarrow M_{m, n}$, tal que para cada $x$ em uma vizinhança $U$ da origem, $F(x) \in M_{m, n}$.

Indicaremos por $M_{m, n}\left(\mathcal{O}_{N}\right)=\left\{F:\left(\mathbb{C}^{N}, 0\right) \rightarrow M_{m, n} \mid F\right.$ é um germe analítico complexo $\}$.

Seja $\mathcal{R}$ o grupo de mudanças de coordenadas em $\left(\mathbb{C}^{N}, 0\right)$, ou seja $\mathcal{R}=\left\{\phi:\left(\mathbb{C}^{N}, 0\right) \rightarrow\right.$ $\left(\mathbb{C}^{N}, 0\right) / \phi$ é um germe de difeomorfismo analítico $\}$.

Definição 2.12. (O Grupo $\mathcal{G}(n, m)$ ) Seja $G L_{i}\left(\mathcal{O}_{N}\right)$ o grupo das matrizes $i \times i$ invertíveis com entradas em $\mathcal{O}_{N}$. Denotemos por $\mathcal{H}=G l_{n}\left(\mathcal{O}_{N}\right) \times G l_{m}\left(\mathcal{O}_{N}\right)$

Então definimos $\mathcal{G}(n, m)=\mathcal{R} \times \mathcal{H}$, o qual é um grupo com as seguintes operações.

$$
(\phi, R, L) \circ(\psi, S, N)=\left(\phi \circ \psi,\left(\psi^{*} R\right) \cdot S,\left(\psi^{*} L\right) \cdot N\right)
$$

onde $\psi^{*}(M)$ é a matriz gerada aplicando $\psi$ em cada uma das entradas de $M$. 
Definimos a ação de $\mathcal{G}(n, m)$ sobre $M_{m, n}\left(\mathcal{O}_{N}\right)$

$$
\begin{aligned}
& \Psi: \mathcal{G}(n, m) \times M_{m, n}\left(\mathcal{O}_{N}\right) \longrightarrow M_{m, n}\left(\mathcal{O}_{N}\right) \\
& ((\phi, R, L), F) \quad \longmapsto \quad L^{-1}\left(\phi^{*} F\right) . R .
\end{aligned}
$$

Dizemos que $F_{1}, F_{2}$ são $\mathcal{G}(n, m)$-equivalentes se $\exists(\phi, R, L) \in \mathcal{G}(n, m)$ tal que $F_{2}=L^{-1}\left(\phi^{*} F_{1}\right) R$.

A ação do grupo $\mathcal{G}(n, m)$ é dada por mudanças de coordenadas na fonte e transformações de linhas e colunas da matriz.

Como já definimos uma ação sobre o espaço das matrizes $m \times n$, a primeira tarefa é determinar o espaço tangente sob a ação do grupo $\mathcal{G}(n, m)$.

Definição 2.13. Seja $F:\left(\mathbb{C}^{N}, 0\right) \rightarrow\left(\mathbb{C}^{p}, 0\right)$ um germe analítico, definimos o $\mathcal{O}_{N^{-}}$módulo

$$
\theta(F)=\mathcal{O}_{N}\left\{\frac{\partial}{\partial y_{i}}\right\}_{i=1}^{p}
$$

módulo de campos vetoriais ao longo de F.

Consideremos o espaço das matrizes com entradas em $\mathcal{O}_{N}$ como um $\mathcal{O}_{N}$ módulo. O espaço tangente será considerado como um $\mathcal{O}_{N}$ submódulo de $\theta(F)$.

Teorema 2.14. [35, 16] Seja $F \in M_{m, n}\left(\mathcal{O}_{N}\right)$.

1. O espaço tangente com relação ao grupo $\mathcal{R}$ é dado por:

$$
T \mathcal{R} \cdot F=\mathfrak{m}\left\{\frac{\partial F}{\partial x_{i}}, \forall 1 \leq i \leq N\right\}
$$

onde $\mathfrak{m}$ é o ideal maximal de $\mathcal{O}_{N}$.

Usamos também a notação $J(F)$ para o $\mathcal{O}_{N^{-}}$módulo gerado pelas matrizes da forma

$$
\frac{\partial F}{\partial x_{j}}=\left(\begin{array}{ccc}
\frac{\partial F_{11}}{\partial x_{j}} & \cdots & \frac{\partial F_{1 n}}{\partial x_{j}} \\
\vdots & \ddots & \vdots \\
\frac{\partial F_{m 1}}{\partial x_{j}} & \cdots & \frac{\partial F_{m n}}{\partial x_{j}}
\end{array}\right)_{m \times n}
$$

$\forall 1 \leq j \leq N$

2. O espaço tangente com relação ao grupo $\mathcal{H}$ é dado por:

$$
T \mathcal{H} \cdot F=\mathcal{O}_{N}\left\{(A F+F B) / A \in M_{m}\left(\mathcal{O}_{N}\right), B \in M_{n}\left(\mathcal{O}_{N}\right)\right\} .
$$


3. O espaço tangente com relação ao grupo $\mathcal{G}(n, m)=\mathcal{R} \times \mathcal{H}$ é dado pela soma dos espaços tangentes:

$$
T \mathcal{G}(n, m) \cdot F=\mathfrak{m}\left\{\frac{\partial F}{\partial x_{i}}, \forall 1 \leq i \leq N\right\}+\mathcal{O}_{N}\left\{(A F+F B) / A \in M_{m}\left(\mathcal{O}_{N}\right), B \in M_{n}\left(\mathcal{O}_{N}\right)\right\} .
$$

Definição 2.15. O espaço tangente estendido para $F \in M_{m, n}\left(\mathcal{O}_{N}\right)$ é dado por:

$$
T_{e} \mathcal{G}(n, m) \cdot F=\mathcal{O}_{N}\left\{\langle J M(F), A F+F B\rangle / A \in M_{m}\left(\mathcal{O}_{N}\right), B \in M_{n}\left(\mathcal{O}_{N}\right)\right\}
$$

Lema 2.16. [35] Seja $F \in M_{m, n}\left(\mathcal{O}_{N}\right)$ com entradas no ideal maximal $\mathfrak{m}$. Então o módulo normal é dado por:

$$
N_{F}=\frac{\theta(F)}{T \mathcal{H} \cdot F}
$$

Definição 2.17. A codimensão do espaço tangente estendido de $G(n, m)$ em $F$ é denominada número de Tjurina de $X=F^{-1}\left(M_{m, n}^{t}\right)$. Isto é,

$$
\tau(F)=\operatorname{dim}_{\mathbb{C}} \frac{\theta(F)}{T_{e} \mathcal{G}(n, m) \cdot F}
$$

\subsubsection{A $\mathcal{K}_{V}$ - Equivalência}

Nesta seção apresentamos o grupo $\mathcal{K}_{V}$ que é um subconjunto do grupo de contato $\mathcal{K}$, que depende do conjunto analítico $(V, 0) \subset\left(\mathbb{C}^{p}, 0\right)$ e age sobre o espaço de funções $F: \mathbb{C}^{N} \rightarrow \mathbb{C}^{p}$. Para nosso contexto $\mathbb{C}^{p}$ é o espaço das matrizes $M_{m, n}$. As referências para esta subseção são [12, 36].

Denotemos por $\theta_{p}$ o $\mathcal{O}_{p}$ módulo de germes de campos vetoriais holomorfos sobre $\mathbb{C}^{p}, 0 \mathrm{e}$ por $\mathcal{K}$ o grupo de contato que age sobre o espaço das funções $F: \mathbb{C}^{N} \rightarrow \mathbb{C}^{p}$.

Definição 2.18. Seja $(V, 0) \subset\left(\mathbb{C}^{p}, 0\right)$ um germe de conjunto analítico. Seja $I(V) \subset \mathcal{O}_{p}$ o ideal de germes que se anulam sobre $V$. Definimos o módulo de germes de campos vetoriais que são tangentes $a \mathrm{~V}$.

$$
\operatorname{Derlog}(V)=\left\{\eta \in \theta_{p} \mid \eta(I(V)) \subset I(V)\right\}
$$

Definição 2.19. Seja $(V, 0) \subset\left(\mathbb{C}^{p}, 0\right)$ um germe de espaço analítico complexo, definido pelos zeros de uma função $H: \mathbb{C}^{p} \rightarrow \mathbb{C}^{r}$. Definimos o submódulo do Derlog $(V)$ por

$$
\operatorname{Derlog}(H)=\left\{\eta \in \theta_{p} \mid \eta(H)=0\right\} .
$$


Nesse contexto, se $H$ é uma boa equação que define $V \subset \mathbb{C}^{p}$, então:

$$
\operatorname{Derlog}(V)=\operatorname{Derlog}(H) \oplus \mathcal{O}_{p}\{\zeta\}
$$

o vetor $\zeta$ é chamado campo vetorial de Euler.

Definição 2.20. Seja $(V, 0) \subset\left(\mathbb{C}^{p}, 0\right)$ um germe de variedade analítica. Definimos o subgrupo de $\mathcal{K}$ denotado por $\mathcal{K}_{V}$, que consiste de germes de difeomorfismos de $\mathcal{K}$ que preservam $\mathbb{C}^{N} \times V$, isto é,

$$
\mathcal{K}_{V}=\left\{\Phi \in \mathcal{K} \mid \Phi\left(\mathbb{C}^{N} \times V\right) \subset \mathbb{C}^{N} \times V\right\}
$$

Seja $\left\{\eta_{j}\right\}_{j=1}^{m}$ o conjunto de geradores para o $\operatorname{Der} \log (V)$. Então o espaço tangente estendido do $\mathcal{K}_{V}$ em $F: \mathbb{C}^{N} \rightarrow \mathbb{C}^{p}$ é

$$
T \mathcal{K}_{V, e} \cdot F=\mathcal{O}_{\mathbb{C}^{N}, 0}\left\{\frac{\partial F}{\partial x_{i}}\right\}_{i=1}^{N}+\mathcal{O}_{\mathbb{C}^{N}, 0}\left\{\eta_{i} \circ F\right\}_{j=1}^{m}
$$

Então a codimensão de $\mathcal{K}_{V}$ em $F$ é a dimensão do espaço normal de $\mathcal{K}_{V}$ em $F$.

$$
\mathcal{K}_{V, e}-\operatorname{codim}(F)=\operatorname{dim}_{\mathbb{C}} \frac{\theta(F)}{T \mathcal{K}_{V, e} \cdot F}
$$

\subsubsection{A $\mathcal{K}_{H}$ - Equivalência}

Seja $(V, 0) \subset\left(\mathbb{C}^{p}, 0\right)$ tal que $V=H^{-1}(0)$, com $H:\left(\mathbb{C}^{p}, 0\right) \rightarrow\left(\mathbb{C}^{r}, 0\right)$ um germe analítico. Neste caso definimos o grupo $\mathcal{K}_{H}$ que consiste de difeomorfismos que preservam todos os conjuntos de nível de $H$, ver [36].

Definição 2.21. Com as hipóteses acima, seja $\pi_{2}: \mathbb{C}^{N} \times \mathbb{C}^{p} \rightarrow \mathbb{C}^{p}$ a projeção sobre o segundo fator. Então o grupo $\mathcal{K}_{H}$ é definido por:

$$
\mathcal{K}_{H}=\left\{\Phi \in \mathcal{K} \mid H \circ \pi_{2} \circ \Phi=H \circ \pi_{2}\right\}
$$

O espaço tangente estendido do grupo $\mathcal{K}_{H}$ em $F:\left(\mathbb{C}^{N}, 0\right) \rightarrow\left(\mathbb{C}^{p}, 0\right)$ é um submódulo de $\theta(F)$, dado por

$$
T \mathcal{K}_{H, e} \cdot F=\mathcal{O}_{\mathbb{C}^{N}, 0}\left\{\frac{\partial F}{\partial x_{i}}\right\}_{i=1}^{N}+\mathcal{O}_{\mathbb{C}^{N}, 0}\left\{\eta_{j} \circ F\right\}_{j=1}^{m}
$$

onde $\left\{\eta_{j}\right\}_{j=1}^{m}$ é o conjunto de geradores para o $\operatorname{Derlog}(H)$.

A codimensão estendida de $\mathcal{K}_{H}$ em $F$ é definida por

$$
T \mathcal{K}_{H, e}-\operatorname{codim}(F)=\operatorname{dim}_{\mathbb{C}} \frac{\theta(F)}{T \mathcal{K}_{H, e} \cdot F}
$$




\subsection{Determinação Finita}

Seja $G$ qualquer um dos grupos definidos acima, $G=\mathcal{G}(n, m), \mathcal{K}_{V}$ ou $\mathcal{K}_{H}$. Nesta seção apresentaremos critérios de determinação finita do grupo $G$.

Definição 2.22. Seja $F \in M_{m, n}\left(\mathcal{O}_{N}\right)$. Dizemos que $F$ é $k-G$ determinado se para toda matriz $P \in M_{m, n}\left(\mathcal{O}_{N}\right)$ tal que $j^{k}(F)=j^{k}(P)$ se tem que $F \sim_{G} P$.

Dizemos que $F$ é finitamente determinado se $\exists k \in \mathbb{N}$ tal que $F$ é $k$-G determinado.

Nos resultados seguintes, apresentamos condições necessárias e suficientes para a $G$-determinação finita.

Proposição 2.23. [34 Seja $F \in M_{m, n}\left(\mathcal{O}_{N}\right)$. Se $F$ é $k$ - $G$ determinado então $\mathfrak{m}^{k+1} \theta(F) \subseteq$ $T G \cdot F$

Teorema 2.24. [34] (C] (Critério Infinitesimal da determinação Finita.) Seja $F \in M_{m, n}\left(\mathcal{O}_{N}\right)$ um germe de matriz. Seja $k>0$ um inteiro tal que se $G$ satisfaz as relações para os grupos $G=\mathcal{G}(n, m), \mathcal{K}_{V}$ e $\mathcal{K}_{H}$ respetivamente

$$
\begin{gathered}
\mathfrak{m}^{k+1} \theta(F) \subset \mathfrak{m}^{2}\left(\frac{\partial F}{\partial x_{1}}, \ldots \frac{\partial F}{\partial x_{N}}\right)+\mathfrak{m}\left\{(A F+F B) / A \in M_{m}\left(\mathcal{O}_{N}\right), B \in M_{n}\left(\mathcal{O}_{N}\right)\right\} \\
\mathfrak{m}^{k+1} \theta(F) \subset \mathfrak{m}^{2}\left(\frac{\partial F}{\partial x_{1}}, \ldots \frac{\partial F}{\partial x_{N}}\right)+\mathfrak{m} D \operatorname{Derlog}(V) . \\
\mathfrak{m}^{k+1} \theta(F) \subset \mathfrak{m}^{2}\left(\frac{\partial F}{\partial x_{1}}, \ldots \frac{\partial F}{\partial x_{N}}\right)+\mathfrak{m} D \operatorname{Derlog}(H) .
\end{gathered}
$$

Então $F$ é $G$ k-finitamente determinado.

O seguinte resultado é uma caracterização geométrica dos germes finitamente determinados.

Definição 2.25. Um germe $F:\left(\mathbb{C}^{N}, 0\right) \rightarrow M_{m, n}$ é $G$ - estável se $T G_{e} \cdot F=\theta(F)$.

Teorema 2.26. [34] (Critério Geométrico da G-determinação Finita.) $F:\left(\mathbb{C}^{N}, 0\right) \rightarrow M_{m, n}$ é $G$-finitamente determinado se e somente se existe um representante $F: U \rightarrow M_{m, n}$ tal que para todo $x \in U-\{0\}$, o germe de $F$ no ponto $x$ é G estável.

Podemos usar o critério geométrico da $G$ - determinação finita para relacionar esta abordagem com as EIDS definidas na seção anterior no caso de variedades determinantais definidas pelo anulamento dos menores maximais da matriz $F(x)$.

Seja $F:\left(\mathbb{C}^{N}, 0\right) \rightarrow M_{m, n}$ e $X=F^{-1}\left(M_{m, n}^{m}\right)$ a variedade determinantal definida por $F$.

Teorema 2.27. $F: \mathbb{C}^{N} \rightarrow M_{m, n}$ é $\mathcal{G}(n, m)$ finitamente determinada se e somente se $X$ é uma EIDS. 
Demonstração: Se $F$ é $\mathcal{G}(n, m)$ finitamente determinado, existe um representante $F: U \rightarrow$ $M_{m, n}, U$ uma vizinhança da origem tal que, $\forall x \in U \backslash\{0\}, F$ é transversal aos estratos $M_{m, n}^{i} \backslash M_{m, n}^{i-1}$ de $M_{m, n}^{m}$. Ou seja, $X=F^{-1}\left(M_{m, n}^{m}\right)$ é uma EIDS. Evidentemente a recíproca também se verifica.

Um germe $F:\left(\mathbb{C}^{N}, 0\right) \rightarrow M_{m, n} \mathcal{G}(n, m)$ finitamente determinado admite um desdobramento versal a $s$-parâmetros (onde $s=\tau(F)$ ), ver [34]

$$
\begin{aligned}
\mathcal{F}:\left(\mathbb{C}^{N} \times \mathbb{C}^{s}, 0\right) & \rightarrow M_{m, n} \\
(x, u) & \mapsto \mathcal{F}(x, u)=\left(\mathcal{F}_{i j}(x, u)\right) .
\end{aligned}
$$

Para um representante $\mathcal{F}: W \times D \rightarrow M_{m, n}$ de $\mathcal{F}$, onde $W$ é uma vizinhança de 0 em $\mathbb{C}^{N}$ e $D$ é vizinhança de 0 em $\mathbb{C}^{s}$, definimos o conjunto de bifurcação $\mathcal{B}(\mathcal{F})$ por

$$
\mathcal{B}(\mathcal{F})=\left\{u \in D \mid \mathcal{F}_{u}: W \rightarrow M_{m, n} \text { não é } \mathcal{G}(n, m) \text { estável }\right\}
$$

onde $\mathcal{F}_{u}: W \rightarrow M_{m, n}, \mathcal{F}_{u}(x)=\mathcal{F}(x, u)$. Este é um subconjunto analítico próprio de $W$ e portanto seu complemento é conexo.

Assim, para cada $u \in D \backslash \mathcal{B}(\mathcal{F}), \mathcal{F}_{u}$ é $\mathcal{G}(n, m)$ - estável. Além disso, $\forall u, u^{\prime} \in D \backslash \mathcal{B}(\mathcal{F})$, $\mathcal{F}_{u} \sim_{\mathcal{G}(n, m)} \mathcal{F}_{u}^{\prime}$

Em outras palavras, $\forall u \in D \backslash \mathcal{B}(\mathcal{F}), \mathcal{F}_{u}$ é uma suavização essencial de $F$. Além disso, quaisquer duas suavizações essenciais são equivalentes.

\subsection{Variedades Cohen-Macaulay de Codimensão 2}

O Teorema de Hilbert-Burch nos permite enunciar a seguinte proposição, que diz que todo germe Cohen-Macaulay de codimensão 2 pode ser expresso como os menores maximais de uma matriz $M$ de tamanho $n \times(n+1)$ e viceversa. No mesmo contexto, deformações de um germe Cohen-Macaulay de codimensão 2 podem ser representadas por perturbações da matriz $M$, e qualquer perturbação da matriz surge como uma deformação do germe $X$.

Dada uma matriz $F \in M_{n(n+1)}\left(\mathcal{O}_{N}\right)$, denotemos por $\Delta_{i}$ o menor $n \times n$ cujo índice $i$, indica que a i-ésima linha da matriz $F(x)$ foi eliminada para calcular o menor, denotemos por $\langle\Delta\rangle=\left\langle\Delta_{1}, \Delta_{2}, \cdots, \Delta_{n+1}\right\rangle$ o ideal gerado pelos menores $\Delta_{i}, i=1, \ldots, n+1$ e por $V(\Delta)$ a variedade em $\mathbb{C}^{N}$ definida pelos zeros das funções $\Delta_{i}$.

Proposição 2.28. [15, página 496]

1. Se a codim $V(\langle\Delta\rangle) \geqslant 2$, a seguinte seqüência 


$$
0 \longrightarrow \mathcal{O}_{N}^{n} \stackrel{F}{\longrightarrow} \mathcal{O}_{N}^{n+1} \stackrel{\Delta}{\longrightarrow} \mathcal{O}_{N} \longrightarrow \frac{\mathcal{O}_{N}}{\langle\Delta\rangle} \longrightarrow 0
$$

é exata. Além disso, $\frac{\mathcal{O}_{N}}{\langle\Delta\rangle}$ é Cohen - Macaulay e codim $V(\langle\Delta\rangle)=2$.

2. Se $X \subset \mathbb{C}^{N}$ é Cohen-Macaulay, tal que $\operatorname{codim}(X)=2$ e $X=V(I)$ então $\mathcal{O}_{N} / I$ tem uma resolução livre minimal do tipo.

$$
0 \longrightarrow \mathcal{O}_{N}^{n} \stackrel{F}{\longrightarrow} \mathcal{O}_{N}{ }^{n+1} \stackrel{I}{\longrightarrow} \mathcal{O}_{N} \longrightarrow \frac{\mathcal{O}_{N}}{I} \longrightarrow 0
$$

Além disso existe uma unidade $u \in \mathcal{O}_{N}$ tal que $I=u . \Delta$, onde $\Delta=\left\langle\Delta_{1}, \ldots, \Delta_{n+1}\right\rangle$.

3. Qualquer perturbação da matriz $F(x)$ dá origem a uma deformação do $X$.

4. Qualquer deformação da variedade X pode ser gerada por uma perturbação da matriz $F(x)$.

Observação 2.29. $X \subseteq \mathbb{C}^{N}$ é chamado Cohen-Macaulay se o anel local $\mathcal{O}_{N} / I$, é CohenMacaulay. 


\section{Topologia de Variedades com Singularidade Isolada}

Vamos estudar neste capítulo a topologia de variedades determinantais isoladas. Estas variedades podem admitir suavização. A topologia da fibra genérica é um invariante da variedade determinantal. Definimos o número de Milnor com base nos trabalhos [12, 35, 31] e a característica de Euler evanescente com base nos trabalhos [12, 31] e estudamos uma fórmula de tipo Lê-Greuel que se verifica para essas variedades.

\subsection{Teoria de Morse}

Seja $(X, 0) \subset\left(\mathbb{C}^{N}, 0\right)$ uma variedade $d$-dimensional com singularidade isolada na origem. Uma suavização de $X$ é uma deformação plana com a propriedade que suas fibras genéricas são suaves.

Definição 3.1. Dizemos que uma variedade $(X, 0) \subset\left(\mathbb{C}^{N}, 0\right) d$-dimensional com singularidade isolada admite uma suavização, se existem uma bola aberta $B_{\epsilon}(0) \subset \mathbb{C}^{N}$ centrada na origem, um subespaço fechado $\mathcal{X} \subset B_{\epsilon}(0) \times D$, onde $D \subset \mathbb{C} e ́$ um disco aberto centrado no zero e uma aplicação analítica própria $\pi: \mathcal{X} \rightarrow D \subset \mathbb{C}$, que é a restrição da aplicação projeção $\pi: B_{\epsilon}(0) \times D \rightarrow D$ tal que:

1. Té plana

2. $\left(\pi^{-1}(0), 0\right)$ é isomorfo a $(X, 0)$

3. $\pi^{-1}(s)$ é não singular para $s \neq 0$

Segue da definição que $\mathcal{X}$ tem singularidade isolada na origem e é uma variedade normal se $X$ é normal em zero. Além disso,

$$
\left.\pi\right|_{\pi^{-1}(D-\{0\})}: \pi^{-1}(D-\{0\}) \rightarrow D-\{0\}
$$


é um fibrado cujas fibras $X_{t}=\pi^{-1}(s)$ são suaves.

Seja $(X, 0) \subset\left(\mathbb{C}^{N}, 0\right)$ uma variedade $d$-dimensional com singularidade isolada na origem. Suponhamos que exista uma suavização para $X$, isto é, uma família plana $\pi: \mathcal{X} \rightarrow \mathbb{C}$, restrição de $\pi: \mathbb{C}^{N} \times \mathbb{C}$, tal que $X_{s}=\pi^{-1}(s)$ é suave para $s \neq 0$ e $X=X_{0}$. Notemos também que $\mathcal{X}$ tem singularidade isolada na origem. Seja $p$ uma função analítica complexa definida em $X$, com singularidade isolada na origem. Seja

$$
\begin{aligned}
\tilde{p}: \mathcal{X} \subset \mathbb{C}^{N} \times \mathbb{C} & \rightarrow \mathbb{C} \\
(x, s) & \mapsto \tilde{p}(x, s)
\end{aligned}
$$

tal que $\tilde{p}(x, 0)=p(x)$ e tal que para todo $s \neq 0, \tilde{p}(., s)=p_{s}$ seja uma função de Morse em $X_{s}$. O número de pontos críticos de $p_{s}$ é finito.

Proposição 3.2. [35] Sejam X uma variedade d-dimensional com singularidade isolada na origem que admite uma suavização $\pi$ e $p_{s}=\tilde{p}(., s)$ como acima. Então:

1. Se $s \neq 0$

$$
X_{s} \cong p_{s}^{-1}(0) \cup\{\text { Células de dimensão } d\},
$$

onde $\cup$ indica a colagem dos dois espaços $e \cong$ indica que os espaços têm o mesmo tipo de homotopia.

2. Temos a igualdade

$$
\chi\left(X_{s}\right)=\chi\left(p_{s}^{-1}(0)\right)+(-1)^{d} n_{\sigma}
$$

onde $n_{\sigma}$ é o número de pontos críticos de $p_{s}$ e $\chi\left(X_{s}\right)$ é a característica de Euler de $X_{s}$.

Na fórmula (3.1) podemos substituir $n_{\sigma}$ pela multiplicidade polar $m_{d}(X)$, que definimos a seguir.

Definição 3.3. [20] Sejam $\mathcal{X} \subset \mathbb{C}^{N} \times \mathbb{C}^{s}$ uma variedade analítica complexa de dimensão $d+s$ e $\pi: \mathcal{X} \rightarrow \mathbb{C}^{s}$ uma função analítica tal que $\pi^{-1}(0)=X$. Seja $\tilde{p}: \mathcal{X} \subset\left(\mathbb{C}^{N} \times \mathbb{C}^{s}, 0\right) \rightarrow\left(\mathbb{C}^{s}, 0\right)$ tal que $\left.\widetilde{p}\right|_{X}$ tem singularidade isolada na origem. Denotamos $m_{d}(X, \pi, \widetilde{p})=m_{0}\left(P_{d}(X, \pi, \widetilde{p})\right)$, onde $P_{d}(X, \pi, \widetilde{p})$ é a d- ésima variedade polar de $\mathcal{X}$ com relação a $\pi$. Em geral $m_{d}(X, \pi, \widetilde{p})$ depende das escolhas de $\mathcal{X}$ e $\tilde{p}$, mas quando $\mathcal{X} \subset \mathbb{C}^{N} \times \mathbb{C}$ é uma suavização única de $X$, $m_{d}(X, \pi, \widetilde{p})$ depende apenas de $\widetilde{p}$. Quando $\widetilde{p}$ é uma projeção genérica, então $m_{d}(X, \pi, \widetilde{p})$ depende apenas de $X$, e é portanto um invariante de $X$ que será denotado por $m_{d}(X)=$ $m_{d}(X, \pi, \widetilde{p})$.

A prova da proposição abaixo pode ser encontrada em [34].

Proposição 3.4. Sob as condições da Proposição $3.2 n_{\sigma}=m_{d}(X)$. 
No estudo da topologia de variedades determinantais com singularidade isolada, os seguintes resultados são úteis.

Teorema 3.5. [22] Seja $(X, 0)$ um espaço analítico complexo d-dimensional, com singularidade isolada e $X_{s}$ a suavização de $(X, 0)$. Então $\pi_{i}\left(X_{s}\right)=0$ para $i \leqslant \operatorname{dim} X-\operatorname{codim} X$.

Denotemos por $b_{i}\left(X_{s}\right)$ o i-ésimo número de Betti da fibra genérica.

Teorema 3.6. [22] Seja $X_{s}$ a suavização de uma singularidade normal, então $b_{1}\left(X_{s}\right)=0$.

Exemplo 3.7. (Interseções completas com singularidade isolada) Seja $(X, 0) \subset\left(\mathbb{C}^{N}, 0\right)$ uma ICIS d-dimesional. A fibra de Milnor $X_{s}$, tem o tipo de homotopia de um bouquet de esferas. $O$ número de esferas é o número de Milnor, denotado por $\mu(X)$ e temos a seguinte relação

$$
\chi\left(X_{s}\right)=1+(-1)^{d} \mu(X) .
$$

\subsection{Funções Definidas em IDS}

Nem toda IDS tem suavização, como vimos no Teorema 2.8. A condição é que $N<$ $(n-t+2)(m-t+2)$. Em [31, 33], J. Nuño Ballesteros, B. Oréfice-Okamoto e J. Tomazella construiram suavizações de $(X, 0)$, no seguinte sentido:

Dado um germe de variedade determinantal $(X, 0)=\left(F^{-1}\left(M_{m, n}^{t}\right), 0\right) \subset\left(\mathbb{C}^{N}, 0\right)$, onde $F: \mathbb{C}^{N} \rightarrow M_{m, n}$ vamos construir uma suavização de $(X, 0)$ através de uma deformação de $F$. Seja $A=\left(a_{i j}\right)_{m n}$ com $a_{i j} \in \mathbb{C}$ números genéricos. Denotamos

$$
\begin{aligned}
F_{A}: \mathbb{C}^{N} & \rightarrow M_{m, n} \\
x & \mapsto F(x)+A
\end{aligned}
$$

e $X_{A}=F_{A}^{-1}\left(M_{m, n}^{t}\right)$.

Lema 3.8. [31 Lema 3.1] Seja $(X, 0)=\left(F^{-1}\left(M_{m, n}^{t}\right), 0\right) \subset\left(\mathbb{C}^{N}, 0\right)$ uma IDS, com $N<(n-$ $t+2)(m-t+2)$. Escolhemos um representante suficientemente pequeno de $X=F^{-1}\left(M_{m, n}^{t}\right)$. Existe um aberto de Zariski não vazio $\mathcal{W} \subset M_{m, n}$ tal que se $A \in \mathcal{W}$ então $X_{A}$ é suave $e$ $\operatorname{rank} F_{A}(x)=t-1$ para todo $x \in X_{A}$.

Teorema 3.9. [33 Teorema 4.14] Sejam $(X, 0)=\left(F^{-1}\left(M_{m, n}^{t}\right), 0\right) \subset\left(\mathbb{C}^{N}, 0\right)$ uma IDS e $A=\left(a_{i j}\right)_{m \times n}$ uma matriz genérica. Definimos

$$
\begin{aligned}
F_{s}: \mathbb{C}^{N} & \rightarrow M_{m, n} \\
x & \mapsto F(x)+s A .
\end{aligned}
$$

Então $X_{s}=F_{s}^{-1}\left(M_{m, n}^{t}\right)$ é uma suavização de $X$. 
Lema 3.10. [33. Lema 5.2] Existe uma função linear $p: \mathbb{C}^{N} \rightarrow \mathbb{C}$ e um conjunto Zariski aberto $\mathcal{W} \subset M_{m, n}$ tal que para todo $A \in \mathcal{W}, X_{A}$ é suave e $\left.p\right|_{X_{A}}$ é uma função de Morse.

Estes resultados foram mostrados usando o fato que para $A$ genérica, $F_{s}$ é transversal aos estratos da variedade determinantal genérica $M_{m, n}^{t}$.

\subsection{Fórmula de Tipo Lê-Greuel para Variedades Determi- nantais}

No estudo da topologia de interseções completas com singularidades isoladas (ICIS), um resultado importante é a fórmula de Lê-Greuel. Esta fórmula estabelece um método indutivo que permite calcular o número de Milnor da variedade, no seguinte sentido:

Sejam $f=\left(f_{1}, \ldots, f_{k-1}, f_{k}\right):\left(\mathbb{C}^{N}, 0\right) \rightarrow\left(\mathbb{C}^{k}, 0\right)$ e $g=\left(f_{1}, \ldots, f_{k-1}\right):\left(\mathbb{C}^{N}, 0\right) \rightarrow$ $\left(\mathbb{C}^{k-1}, 0\right)$. Seja $X=g^{-1}(0)$ e $Y=f^{-1}(0)=X \cap f_{k}^{-1}(0)$, então

$$
\mu(X, 0)+\mu(Y, 0)=\operatorname{dim}_{\mathbb{C}} \frac{\mathcal{O}_{N}}{\left\langle f_{1}, \ldots, f_{k-1}, J\left(f_{1}, \ldots, f_{k}\right)\right\rangle}
$$

onde $J\left(f_{1}, \ldots, f_{k}\right)$ é o ideal de todos os menores $k \times k$ da matriz jacobiana de $f$. A dimensão descrita na parte direita é igual ao número de pontos críticos de uma perturbação de $f_{k}$ restrita à fibra genérica $g$.

Fórmulas de tipo Lê- Greuel para variedades determinantais foram estudadas recentemente em [31, 35, 12]. Para superfícies determinantais em $\mathbb{C}^{N}$, segue do Teorema 3.6 que $b_{1}\left(X_{s}\right)=0$. Então a seguinte definição se justifica.

Definição 3.11. [35] Sejam X uma superfície determinantal em $\mathbb{C}^{N}$, com singularidade isolada na origem. O número de Milnor de $X$, denotado por $\mu(X)$, é o segundo número de Betti de $X_{s}$,

$$
\mu(X, 0)=b_{2}\left(X_{s}\right)
$$

Para obter a fórmula de tipo Lê-Greuel, é considerada a curva determinantal obtida pela interseção de $X$ com um hiperplano $H$, definido pelo núcleo de uma aplicação linear $p$, tal que $X \cap H$ tem singularidade isolada na origem.

O seguinte resultado é encontrado no artigo [35], para o caso de superfícies $X \subset \mathbb{C}^{4}$, mas vale também para superfícies com singularidade isolada em $\mathbb{C}^{N}$, que admitem suavização.

Proposição 3.12. 35 Seja $(X, 0) \subset\left(\mathbb{C}^{N}, 0\right)$ o germe de uma superficie determinantal com singularidade isolada na origem. Seja $p:\left(\mathbb{C}^{N}, 0\right) \rightarrow(\mathbb{C}, 0)$ uma aplicação linear cuja restrição à X tem singularidade isolada na origem. Então: 


$$
\mu(X)+\mu(X \cap H)=m_{2}(X) .
$$

Para dimensões $d=\operatorname{dim} X>2$, os números de Betti $b_{i}(X), 2 \leqslant i<d$ não são necessariamente nulos, neste caso em [31, 12] os autores definem a característica de Euler evanescente de variedadades que admitem suavizações. No trabalho [12] a noção de característica de Euler evanescente é também definida para EIDS que não são necessariamente IDS, ver Capítulo6.

Definição 3.13. [31] Seja $(X, 0) \subset\left(\mathbb{C}^{N}, 0\right)$ uma IDS tal que $N<(n-t+2)(m-t+2)$. A característica de Euler evanescente é definida por

$$
\nu(X, 0)=(-1)^{d}\left(\chi\left(X_{s}\right)-1\right)
$$

onde $X_{s}$ é uma suavização de $X$ e $\chi\left(X_{s}\right)$ é a característica de Euler de $X_{t}$.

Observação 3.14. Se $\tilde{\chi}(X)=\chi\left(X_{s}\right)-1$ então $\tilde{\chi}(X)=(-1)^{d} \nu(X)$.

Teorema 3.15. [31] Seja $(X, 0) \subset\left(\mathbb{C}^{N}, 0\right)$ uma IDS tal que $N<(n-t+2)(m-t+2)$ e seja $p:\left(\mathbb{C}^{N}, 0\right) \rightarrow(\mathbb{C}, 0)$ uma função linear genérica cuja restrição à $X$ tem singularidade isolada na origem. Então,

$$
\nu(X)+\nu\left(X \cap p^{-1}(0)\right)=m_{d}(X)
$$

Observação 3.16. Quando $d=2$, então $\nu(X)=\mu(X)$.

Exemplo 3.17. [34] Seja $X=F^{-1}\left(M_{2,3}^{2}\right) \subset \mathbb{C}^{4}$ definida pela função:

$$
\begin{aligned}
F: \mathbb{C}^{4} & \rightarrow \\
(x, y, z, w) & \mapsto\left(\begin{array}{ccc}
w & M_{2,3} \\
z & y & x \\
z & w & y
\end{array}\right)
\end{aligned}
$$

Considere $X_{s}$ a suavização de $X$, definida pela matriz

$$
\left(\begin{array}{ccc}
z & y+w & x+s \\
w & x & y
\end{array}\right) .
$$

Considere $p: \mathbb{C}^{4} \rightarrow \mathbb{C}$ dada por $p(x, y, z, w)=w$, como $m_{2}(X)=3$ e $\mu\left(X \cap p^{-1}(0)\right)=2$, então $\mu(X)=1$. 


\section{A Transformada de Nash de Variedades Determinantais}

Estudamos a transformada de Nash da variedade determinantal genérica $M_{m, n}^{t}$, de forma detalhada, seguindo W. Èbeling e S. M. Guser̆n-Zade em [23]. Se verifica que a transformada de Nash de $M_{m, n}^{t}$ é suave e portanto uma resolução para $M_{m, n}^{t}$. Com isso podemos estudar a transformada de Nash $\widehat{X}$, da EIDS $X$, definida por $F$ e obter condições sobre a transversalidade de $F$ aos estratos da estratificação de $M_{m, n}^{t}$ para obter $\widehat{X}$ suave.

\subsection{Resoluções de $M_{m, n}^{t}$}

Nesta seção apresentaremos três tipos de resoluções para as variedades determinantais genéricas, sendo uma delas a transformação de Nash da variedade determinantal genérica. Nosso objetivo aqui é apresentar com mais detalhes os resultados de W. Èbeling e S. M. Guseřn-Zade em [23].

Denotemos por $\Sigma X$ o conjunto dos pontos singulares de $X$ e por $X_{\text {reg }}=X \backslash \Sigma X$ a parte regular de $X$. Consideremos $G r(k, n)$ a variedade grassmanniana de subespaços vetoriais complexos de dimensão $k$ em $\mathbb{C}^{n}$.

No seguinte lema vamos mostrar que o espaço tangente de $\operatorname{Gr}(k, n)$ em um ponto $W$ pode ser identificado com $\operatorname{Hom}\left(W, \frac{\mathbb{C}^{n}}{W}\right)$, o que será usado para calcular o espaço tangente de $M_{m, n}^{t}$.

Lema 4.1. Dado $W \in G r(k, n)$, temos que $T_{W}(G r(k, n))$ e Hom $\left(W, \frac{\mathbb{C}^{n}}{W}\right)$ são isomorfos.

Demonstração: Definimos a seguinte aplicação linear de espaços vetoriais:

$$
\begin{array}{ccc}
L: \operatorname{Hom}\left(W, \mathbb{C}^{n}\right) & \rightarrow & T_{W}(G r(k, n)) \\
\phi & \mapsto & W_{\phi, \epsilon}
\end{array}
$$


como segue: se $w_{1}, \ldots, w_{k} \in W \subset \mathbb{C}^{n}$ geram uma base de $W$, então definimos $W_{\phi, \epsilon}=\left\langle w_{1}+\right.$ $\left.\epsilon \phi\left(w_{1}\right), \ldots, w_{k}+\epsilon \phi\left(w_{k}\right)\right\rangle$. Notemos que os vetores $w_{i}+\epsilon \phi\left(w_{i}\right), i=1, \ldots, k$ são ainda linearmente independentes pois são pequenas perturbações de um conjunto lineramente independente.

Vamos mostrar que $L$ é sobrejetora: suponha que $w_{1}, \cdots, w_{k}, e_{k+1}, \cdots, e_{n}$ formem uma base de $\mathbb{C}^{n}$, completando a base $W$. Cada elemento $W^{\prime} \in G r(k, n)$ perto de $W$ pode ser dado como o gráfico de uma aplicação linear $\lambda: W \rightarrow W^{\perp}$ definida por uma matriz $(n-k) \times k$, $\alpha=\left(b_{i j}\right)$ com relação as bases $\left\langle w_{1}, \ldots, w_{k}\right\rangle$ de $W$ e $\left\langle e_{k+1}, \ldots, e_{n}\right\rangle$ de $W^{\perp}$. Logo $W^{\prime}=$ $\{(w, \lambda(w)) \mid w \in W\}$, assim uma base para $W^{\prime}$ é dada por $\left\langle w_{i}+b_{i, k+1} e_{k+1}+\cdots+b_{i, n} e_{n}\right\rangle$. Então definimos $\phi$ como

$$
\phi\left(w_{i}\right)=b_{i, k+1} e_{i, k+1}+\cdots+b_{i, n} e_{n}
$$

Agora, temos que

$$
\operatorname{Hom}(W, W) \subset \operatorname{Hom}\left(W, \mathbb{C}^{n}\right)
$$

está contido no $\operatorname{Ker}(L)$, pois dado $\phi: W \rightarrow W, L(\phi)=W$ que corresponde ao espaço vetorial 0 em $\mathbb{C}^{N} / W$. Como $L$ é sobrejetora, usando o teorema do posto, obtemos

$$
\operatorname{dim} \operatorname{ker}(L)=\operatorname{dim} H o m\left(W, \mathbb{C}^{n}\right)-\operatorname{dim} G r(k, n)=n k-k(n-k)=k^{2} .
$$

Como $\operatorname{dim} \operatorname{Hom}(W, W)=k^{2}$ e $\operatorname{Hom}(W, W) \subset \operatorname{Ker}(L)$, então

$$
\operatorname{Hom}(W, W)=\operatorname{Ker}(L)
$$

Pelo Teorema do isomorfismo temos:

$$
\operatorname{Hom}\left(W, \frac{\mathbb{C}^{n}}{W}\right) \cong \frac{\operatorname{Hom}\left(W, \mathbb{C}^{n}\right)}{\operatorname{Hom}(W, W)} \cong T_{W}(G r(k, n)) .
$$

Definição 4.2. Dada uma variedade singular $X$, uma resolução de $X$ é uma aplicação $\pi$ : $Y \rightarrow X$, onde $Y$ é uma variedade suave e que satisfaz as seguintes condições:

$-\left.\pi\right|_{\pi^{-1}\left(X_{\text {reg }}\right)}: \pi^{-1}\left(X_{\text {reg }}\right) \rightarrow X-\Sigma X$ é um isomorfismo.

- $\pi$ é própria.

Existem ao menos 4 resoluções para a variedade $M_{m, n}^{t}$. Vamos defini-las e estudar com mais detalhes a resolução de Nash.

1. A primeira resolução é construída da seguinte maneira. Consideremos as matrizes de tamanho $m \times n$ como aplicações $\mathbb{C}^{n} \rightarrow \mathbb{C}^{m}$. Para $t \leqslant \min \{m, n\}$ definimos $Y_{1}$, uma 
subvariedade de $M_{m, n} \times G r(n-t+1, n)$ :

$$
Y_{1}=\left\{(A, W) \in M_{m, n} \times G r(n-t+1, n) / A(W)=0\right\}
$$

Consideramos a aplicação $\pi_{1}: Y_{1} \rightarrow M_{m, n}^{t}$ que é a projeção no primeiro fator. A projeção no segundo fator $\pi_{2}: Y_{1} \rightarrow G r(n-t+1, n)$ define um fibrado vetorial, cuja fibra tem posto $m(t-1)$. Assim $Y_{1}$ é suave e portanto uma resolução de $M_{m, n}^{t}$.

O seguinte lema será util para calcular a transformada de Nash de $M_{m, n}^{t}$. Sua prova usa a resolução $Y_{1}$.

Lema 4.3. [1] Seja $A \in\left(M_{m, n}^{t}\right)_{\text {reg. }}$ O espaço tangente de $\left(M_{m, n}^{t}\right)_{\text {reg }}$ no ponto A é igual $a$ :

$$
T_{A}\left(M_{m, n}^{t}\right)=\left\{B \in M_{m, n} / B . \operatorname{ker} A \subset \operatorname{im} A\right\} .
$$

Demonstração: Consideremos a aplicação $\pi_{1}: Y_{1} \rightarrow M_{m, n}^{t}$ e determinemos o espaço tangente de $(A, W) \in Y_{1}$.

Dado $(A, W) \in Y_{1}$, temos que $A W=0$. O espaço tangente é dado por um par $(B, \phi)$ onde $B$ é uma matriz e $\phi \in \operatorname{Hom}\left(W, \frac{\mathbb{C}^{n}}{W}\right)$ tais que $(A+\epsilon B)\left(w_{i}+\epsilon \phi\left(w_{i}\right)\right)=0$, onde $\left\{w_{i}\right\}$ é uma base de $W$. Assim $A . W=0$ e $A \phi\left(w_{i}\right)+B . w_{i}=0,(A \phi+B) W=0$, $B . W=A(-\phi W)$, i.e. $B . W \subset \operatorname{im}(A)$. Assim

$$
d_{(A, W)} \pi_{1}\left(T_{(A, W)} Y_{1}\right)=\left\{B \in M_{m, n} / B . W \subset \operatorname{im}(A)\right\} .
$$

Quando $A \in\left(M_{m, n}^{t}\right)_{\text {reg }}=M_{m, n}^{t} \backslash M_{m, n}^{t-1}, \operatorname{rank} A=t-1$ então $W=\operatorname{ker} A$ e temos que $(A, \operatorname{ker} A) \in Y_{1}$ e a aplicação $d_{(A, \operatorname{ker} A)} \pi: T_{(A, \operatorname{ker} A)} Y_{1} \rightarrow T_{A} M_{m, n}^{t}$ é injetiva. Assim

$$
T_{A}\left(M_{m, n}^{t}\right)=\left\{B \in M_{m, n} / B \operatorname{ker} A \subset \operatorname{im} A\right\} .
$$

2. A segunda resolução é dada considerando as matrizes de tamanho $m \times n$, como aplicações de $\mathbb{C}^{m} \rightarrow \mathbb{C}^{n}$. Definimos $Y_{2}$ da mesma forma que a primeira resolução dada como um subconjunto de $M_{m, n} \times G r(m-t+1, m)$ e definimos $\pi_{2}: Y_{2} \rightarrow M_{m, n}^{t}$.

3. Uma terceira resolução é definida considerando Y definido por:

$$
Y=\left\{(A, W) \in M_{m, n} \times G r(t-1, m) / \operatorname{im} A \subset W\right\}
$$

e definindo $\pi: Y \rightarrow M_{m, n}^{t}$ como a projeção no primeiro fator.

4. A transformada de Nash da variedade $M_{m, n}^{t}$, denotada por $\widehat{M}_{m, n}^{t}$ é uma resolução da 
variedade $M_{m, n}^{t}$.

Vamos usar esta definição para o caso da variedade analítica das matrizes de posto menor do que $t, M_{m, n}^{t}$. Denotamos por $d_{m, n}^{t}=m n-(m-t+1)(n-t+1)$ a dimensão da variedade $M_{m, n}^{t}$. A transformação de Nash $\widehat{M}_{m, n}^{t}$ é um subespaço de $M_{m, n}^{t} \times G r\left(d_{m, n}^{t}, M_{m, n}\right)$ dado por:

$$
\widehat{M}_{m, n}^{t}=\overline{\left\{(A, T) / A \in M_{m, n}^{t} \backslash M_{m, n}^{t-1}=\left(M_{m, n}^{t}\right)_{r e g}, T=T_{A} M_{m, n}^{t}\right\}} .
$$

Na sequiência daremos uma caracterização da transformada de Nash $M_{m, n}^{t}$. Consideremos a seguinte aplicação:

$$
\begin{aligned}
\alpha: G r(n-t+1, n) \times G r(t-1, m) & \rightarrow G r\left(d_{m, n}^{t}, m n\right) \\
\left(W_{1}, W_{2}\right) & \mapsto\left\{B \in M_{m, n} / B W_{1} \subset W_{2}\right\} .
\end{aligned}
$$

Proposição 4.4. [23] A transformação de Nash de $M_{m, n}^{t}$ é dada por:

$$
\widehat{M}_{m, n}^{t}=\left\{(A, T) / A \in M_{m, n}, T=\alpha\left(W_{1}, W_{2}\right), W_{1} \subset \operatorname{ker} A, \operatorname{im} A \subset W_{2}\right\}
$$

\section{Demonstração:}

Dados $W_{1}$ e $W_{2}, Q=\left\{B \in M_{m, n} / B W_{1} \subset W_{2}\right\}$ é um subespaço vetorial de $M_{m, n}$. De fato, temos:

(a) Seja $B_{1} \in Q, B_{2} \in Q$, então $B_{1} W_{1} \subset W_{2}$ e $B_{2} W_{1} \subset W_{2}, \log$ o $\left(B_{1}+B_{2}\right) W_{1}=$ $B_{1} W_{1}+B_{2} W_{1} \subset W_{2}$.

(b) Seja $k \in \mathbb{C}$ e $B \in Q$ então $(k B) W_{1} \subset k W_{2} \subset W_{2}$.

Os elementos $W_{1} \in G r(n-t+1, n)$ podem ser descritos por coordenadas, dadas por uma matriz complexa $n \times(n-t+1), Y=\left(y_{i j}\right)$ de posto $n-t+1$, cujas colunas $v_{1}=\left(y_{11}, \cdots, y_{1 n}\right), \cdots, v_{n-t+1}=\left(y_{(n-t+1) 1}, \cdots, y_{(n-t+1) n}\right)$ formam uma base de $W_{1}$. Todas as outras bases de $W$ são combinações lineares da base dada, determinadas por uma matriz $A=\left(a_{i j}\right), r \times r$, invertível. Então as coordenadas de $W$ estão definidas por $Y A$, onde $A$ é uma matriz invertível.

Considerando $W_{1}$ gerado pelos primeiros $n-t+1$ elementos da base canônica de $\mathbb{C}^{n}$, $\left\{e_{1}, \cdots, e_{n-t+1}\right\}$, e $W_{2}$ gerado pelos últimos $t-1$ geradores da base canônica de $\mathbb{C}^{m}$, podemos representar $W_{1}, W_{2}$ pelas seguintes matrizes: 


$$
W_{1}=\left(\begin{array}{c}
I d_{(n-t+1) \times(n-t+1)} \\
------- \\
0_{(t-1) \times(n-t+1)}
\end{array}\right)_{n \times(n-t+1)} W_{2}=\left(\begin{array}{c}
0_{(m-t+1) \times(t-1)} \\
------- \\
I d_{(t-1) \times(t-1)}
\end{array}\right)_{m \times(t-1)} .
$$

Dado um elemento $B \in W$, temos que $B W_{1} \subset W_{2}$, então podemos dividir a matriz $B$ em blocos, e fazer o produto:

Considere $B=\left(\begin{array}{c|c}P & Q \\ \hline R & S\end{array}\right)_{m \times n}$, onde $[P]_{(m-t+1)(n-t+1)},[Q]_{(m-t+1)(t-1)},[R]_{(t-1)(n-t+1)}$, $[S]_{(t-1) \times(t-1)}$.

$$
\left(\begin{array}{c|c}
P & Q \\
\hline R & S
\end{array}\right) \cdot\left(\begin{array}{c}
I d \\
--- \\
0
\end{array}\right)=\left(\begin{array}{c}
P_{(m-t+1) \times(n-t+1)} \\
-------- \\
R_{(t-1) \times(n-t+1)}
\end{array}\right)
$$

Como $B W_{1} \subset W_{2}$, então $P=0$. Assim $B=\left(\begin{array}{c|c}0 & Q \\ \hline R & S\end{array}\right)$.

Vamos mostrar que

$$
\begin{aligned}
\alpha: G r(n-t+1, n) \times G r(t-1, m) & \rightarrow G r\left(d_{m, n}^{t}, m n\right) \\
\left(W_{1}, W_{2}\right) & \mapsto\left\{B \in M_{m, n} / B W_{1} \subset W_{2}\right\}
\end{aligned}
$$

é um mergulho.

Sejam $W_{1}, W_{1}^{\prime} \in G r(n-t+1, n)$, e $W_{2}, W_{2}^{\prime} \in G r(t-1, m)$, com $\alpha\left(W_{1}, W_{2}\right)=$ $\alpha\left(W_{1}^{\prime}, W_{2}^{\prime}\right)$. Sem perda de generalidade podemos considerar $W_{1}$ e $W_{2}$, como no caso anterior e $W_{1}^{\prime}, W_{2}^{\prime}$ representados pelas matrizes:

$W_{1}^{\prime}=\left(\begin{array}{c}M \\ --- \\ N\end{array}\right), W_{2}^{\prime}=\left(\begin{array}{c}T \\ --- \\ U\end{array}\right)$.

A injetividade de $\alpha$ vai ser demonstrada em 3 casos:

1) Caso: $W_{2}=W_{2}^{\prime}=\left(\begin{array}{c}0_{(m-t+1) \times(t-1)} \\ ------ \\ I d_{(t-1) \times(t-1)}\end{array}\right)$.

Como $B \in \alpha\left(W_{1}, W_{2}\right)$ então $B$ é da forma $B=\left(\begin{array}{c|c}0 & Q \\ \hline R & S\end{array}\right)$, e como $\alpha\left(W_{1}, W_{2}\right)=$ $\alpha\left(W_{1}^{\prime}, W_{2}^{\prime}\right)$, temos $\left(\begin{array}{c|c}0 & Q \\ \hline R & S\end{array}\right) \cdot\left(\begin{array}{c}M \\ --- \\ N\end{array}\right)=\left(\begin{array}{c}Q N \\ --- \\ R M+S N\end{array}\right)$, os vetores colunas 
pertencem a $W_{2}$. Em termos de matrizes, por abuso de notação, podemos dizer que $\left(\begin{array}{c}Q N \\ --- \\ R M+S N\end{array}\right) \subset\left(\begin{array}{c}0_{(m-t+1) \times(t-1)} \\ ------- \\ I d_{(t-1) \times(t-1)}\end{array}\right)$. Assim $Q N=0$ para toda matriz $Q$, então $N=0, \operatorname{logo} W_{1}^{\prime}=\left(\begin{array}{c}M \\ --- \\ 0\end{array}\right)$. Como $W_{1}^{\prime}$ é um subespaço vetorial temos que $\operatorname{det} M \neq 0$, assim concluímos que $W_{1}=W_{1}^{\prime}$.

2) Caso $W_{2} \neq W_{2}^{\prime}$ e $\operatorname{det} U \neq 0$.

Neste caso, a matriz $U$ em $W_{2}^{\prime}$ é invertível. Então $W_{2}^{\prime}$ pode ser representada por $W_{2}^{\prime}=$ $\left(\begin{array}{c}T \\ --- \\ U\end{array}\right) \cdot U^{-1}=\left(\begin{array}{c}T U^{-1} \\ --- \\ I d\end{array}\right)$

Então $B W_{1}^{\prime}=\left(\begin{array}{c}Q N \\ --- \\ R M+S N\end{array}\right) \subset\left(\begin{array}{c}T U^{-1} \\ --- \\ I d\end{array}\right)$. Como esta condição está satisfeita para toda matriz $Q$, podemos considerar $Q=0$, $\log 0 T U^{-1}=0$, assim $W_{2}=W_{2}^{\prime}$, pelo caso anterior.

3) Caso $W_{2} \neq W_{2}^{\prime}$ e $\operatorname{det} U=0$.

Usando os mesmos argumentos $B W_{1}^{\prime}=\left(\begin{array}{c}Q N \\ --- \\ R M+S N\end{array}\right) \subset\left(\begin{array}{c}T \\ --- \\ U\end{array}\right)$. Como esta condição é verificada para qualquer matriz $Q$, podemos considerar $Q=0$, assim $T=0$. Temos uma contradição, pois o posto de $W_{2}^{\prime}$ é $t-1$.

Continuando com o cálculo da transformação de Nash para a variedade $M_{m, n}^{t}$ e considerando o espaço tangente calculado temos:

$$
\begin{gathered}
\widehat{M}_{m, n}^{t}=\overline{\left\{(A, T) / A \in M_{m, n}^{t} \backslash M_{m, n}^{t-1}=\left(M_{m, n}^{t}\right)_{r e g}, T=T_{A} M_{m, n}^{t}\right\}} \\
\widehat{M}_{m, n}^{t}=\overline{\left\{(A, T) / A \in M_{m, n}^{t} \backslash M_{m, n}^{t-1}=\left(M_{m, n}^{t}\right)_{r e g}, T=\alpha(\operatorname{ker} A, \operatorname{im} A)\right\}}
\end{gathered}
$$

Seja $(A, T) \in \overline{\left\{(A, T) / A \in M_{m, n}^{t} \backslash M_{m, n}^{t-1}=\left(M_{m, n}^{t}\right)_{r e g}, T=T_{A} M_{m, n}^{t}\right\}}$, então $(A, T)=$ $\lim _{r \rightarrow \infty}\left(A_{r}, T_{r}\right), \operatorname{com}\left(A_{r}, T_{r}\right) \in\left\{(A, T) / A \in M_{m, n}^{t} \backslash M_{m, n}^{t-1}=\left(M_{m, n}^{t}\right)_{r e g}, T=T_{A} M_{m, n}^{t}\right\}$ então $T_{r}=\alpha\left(\operatorname{ker} A_{r}, \operatorname{Im} A_{r}\right)$. Como $\alpha$ é um mergulho então 
$\lim T_{r}=\alpha\left(\lim \operatorname{ker} A_{r}, \lim \operatorname{im} A_{r}\right)$. Assim,

$\overline{\left\{A / \operatorname{ker} A=W_{1}, \operatorname{im} A=W_{2}\right\}}=\left\{A \in M_{m, n} / W_{1} \subset \operatorname{ker} A, \operatorname{im} A \subset W_{2}\right\}$.

Mostramos acima que a restrição de $\pi$ ao segundo fator de $\widehat{M}_{m, n}^{t} \subset M_{m, n}^{t} \times G r\left(d_{m, n}^{t}, m n\right)$ é a projeção de um fibrado vetorial de posto $(t-1)^{2}$ e de base $\alpha(G r(n-t+1, n) \times G r(t-1, m))$. A fibra $F$ deste fibrado vetorial é dada por

$$
\pi^{-1}\left(\alpha\left(W_{1}, W_{2}\right)\right)=\left\{A / W_{1} \subset \operatorname{ker} A, \operatorname{im} A \subset W_{2}\right\} .
$$

Temos $\operatorname{dim} B+\operatorname{dim} F=\operatorname{dim} \widehat{M}_{m, n}^{t}, \operatorname{dim} B=(t-1)(n-t+1)+(t-1)(m-t+1) \mathrm{e}$ $\operatorname{dim} \widehat{M}_{m, m}^{t}=\operatorname{dim} M_{m, n}^{t}=m n-(m-t+1)(n-t+1), \operatorname{assim} \operatorname{dim} F=(t-1)^{2} \cdot \operatorname{Logo} \widehat{M}_{m, n}^{t}$ é suave.

Assim obtemos uma resolução de $M_{m, n}^{t}, Y_{3}=\widehat{M}_{m, n}^{t}$ dada pela transformação de Nash e denotamos

$$
\pi_{3}: \widehat{M}_{m, n}^{t} \rightarrow M_{m, n}^{t}
$$

a projeção no primeiro fator.

Exemplo 4.5. Vamos calcular a transformada de Nash de $M_{2,3}^{2}$.

$$
M_{2,3}^{2}=\left\{A=\left(\begin{array}{ccc}
x & y & z \\
w & u & v
\end{array}\right) \mid x u-w y=x v-w z=y v-u z=0\right\} .
$$

Para isso primeiro vamos calcular $\nu^{-1}(A)=\left\{(A, T) \in M_{2,3} \times G r(2,4) \mid T=T_{A}\left(M_{2,3}^{2}\right)_{\text {reg }}\right\}$, onde A é um ponto regular.

Para ilustrar, se consideramos $A=\left(\begin{array}{lll}1 & 0 & 0 \\ 1 & 0 & 0\end{array}\right)$, então

$$
T_{A} M_{2,3}^{2}=\left\{B \in M_{2,3} \mid B \operatorname{ker} A \subset \operatorname{im} A\right\}=\left\{\left(\begin{array}{lll}
a & b & b \\
d & e & e
\end{array}\right) \mid a, b, d, e \in \mathbb{C}\right\}
$$

Temos então $\nu^{-1}(A)=\left\{\left(\left(\begin{array}{lll}1 & 0 & 0 \\ 1 & 0 & 0\end{array}\right),\left(\begin{array}{lll}a & b & b \\ d & e & e\end{array}\right)\right) \mid a, b, d, e \in \mathbb{C}\right\}$.

Seja $A=\left(\begin{array}{lll}0 & 0 & 0 \\ 0 & 0 & 0\end{array}\right)$, ponto singular de $M_{2,3}^{2}$, agora vamos calcular

$$
\nu^{-1}(A)=\left\{(A, T) \in M_{2,3} \times G r(2,4) / T=\alpha\left(W_{1}, W_{2}\right), W_{1} \subset \operatorname{ker} A, \operatorname{im} A \subset W_{2}\right\} .
$$

Temos que $(A, T) \in \widehat{M}_{2,3}^{2}$, se $T=\alpha\left(W_{1}, W_{2}\right) \operatorname{com} W_{1} \subset \operatorname{ker} A=\mathbb{C}^{3},\{0\}=\operatorname{Im} A \subset W_{2}$, 
com $\operatorname{dim} W_{1}=2, \operatorname{dim} W_{2}=1$.

Dado $W_{1} \subset \operatorname{ker} A=\mathbb{C}^{3} e(0)=\operatorname{im} A \subset W_{2}$, se consideramos $W_{1}=\langle(1,0, r),(0,1, s)\rangle$ e $W_{2}=\langle(1, u)\rangle$, com $r, s, u \in \mathbb{C}$, podemos construir uma matriz $C$ tal que $\operatorname{ker} C=W_{1} e$ $\operatorname{im} C=W_{2}$.

Assim obtemos $C$ da seguinte forma:

$$
C=\left(\begin{array}{ccc}
r & s & -1 \\
u r & u s & -u
\end{array}\right)
$$

Com isso podemos concluir que $C \in \nu^{-1}(0)$,

Para as outras bases acontece o mesmo, existe uma matriz com uma entrada distinta de zero.

Logo $\nu^{-1}(0)$ é o conjunto de matrizes de posto 1 módulo o subconjunto $\{c A \mid \operatorname{rank}(A)=$ $1, c \in \mathbb{C}, c \neq 0\}$, tais que $\nu(M)=0$ e $\operatorname{dim} \nu^{-1}(0)=3$.

\subsection{Transformação de Nash de uma EIDS}

Seja $X$ uma variedade determinantal essencialmente isolada (Def. 2.4), ou seja $X=$ $F^{-1}\left(M_{m, n}^{t}\right)$, onde $F$ é transversal a todos os estratos da estratificação canônica de $M_{m, n}^{t}$ fora da origem.

Usando a transformação de Nash para matrizes $M_{m, n}^{t}$, podemos calcular a transformação de Nash para $X=F^{-1}\left(M_{m, n}^{t}\right)$, que denotamos por $\widehat{X} \subset \mathbb{C}^{N} \times G r(d, N)$, onde $d=N-(n-t+$ 1) $(m-t+1)$.

Por definição temos que $\widehat{X}$ é dada por:

$$
\widehat{X}=\overline{\left\{(x, T) / x \in X_{\text {reg }}, T=T_{x}\left(X_{\text {reg }}\right)\right\}}
$$

Usando a transversalidade de $F$ temos que:

$$
\widehat{X}=\overline{\left\{(x, T) / F(x) \in\left(M_{m, n}^{t} \backslash M_{m, n}^{t-1}\right), T=\left(d_{x} F\right)^{-1}\left(T_{F(x)}\left(M_{m, n}^{t} \backslash M_{m, n}^{t-1}\right)\right)\right\}}
$$

Agora vamos considerar os seguintes casos:

1. Se $x \in X_{\text {reg }}=F^{-1}\left(M_{m, n}^{t} \backslash M_{m, n}^{t-1}\right)$. Temos que $(x, T) \in \widehat{X}$, se $F(x) \in M_{m, n}^{t} \backslash M_{m, n}^{t-1}$, então $T=T_{x}\left(X_{\text {reg }}\right)=\left(d_{x} F\right)^{-1}\left(T_{F(x)}\left(M_{m, n}^{t} \backslash M_{m, n}^{t-1}\right)\right)$.

2. Se $x \in \Sigma X=F^{-1}\left(M_{m, n}^{t-1}\right)$.

Temos que $(x, T) \in \widehat{X}$, se existe uma seqüência $\left(x_{r}, T_{r}\right)$ tal que $x_{r} \rightarrow x \in F^{-1}\left(M_{m, n}^{t-1}\right)$, 
$F\left(x_{r}\right) \in\left(M_{m, n}^{t}\right)_{r e g}, \operatorname{com} T_{r}=d_{x_{r}} F^{-1}\left(T_{F\left(x_{r}\right)}\left(M_{m, n}^{t} \backslash M_{m, n}^{t-1}\right)\right)$ e $T_{r} \rightarrow T$, onde $T=$ $\lim _{x_{r} \rightarrow x} T_{r}$.

No próximo teorema obtemos condições para que a transformada de Nash de uma EIDS seja suave.

Teorema 4.6. Seja $X=F^{-1}\left(M_{m, n}^{t}\right) \subset \mathbb{C}^{N}$ uma EIDS, definida por $F: \mathbb{C}^{N} \rightarrow M_{m, n}$. Se $F \dot{e}$ transversal aos limites dos espaços tangentes aos estratos da variedade $M_{m, n}^{t}$ então $\widehat{X}$ é suave.

Inicialmente, mostraremos os seguintes resultados

Proposição 4.7. Seja $X=F^{-1}\left(M_{m, n}^{t}\right) \subset \mathbb{C}^{N}$ uma EIDS, definida pela função $F: \mathbb{C}^{N} \rightarrow$ $M_{m, n}$. Se $F$ é transversal aos estratos da variedade $M_{m, n}^{t}$ então $\widehat{X}$ é suave.

Demonstração: Como $M_{m, n}^{1}=\{0\}$ é estrato de dimensão 0 e $F$ é transversal aos estratos de $M_{m, n}^{t}$, então $F$ é transversal a $M_{m, n}^{1}, \forall x \in \mathbb{C}^{N}$ tal que $F(x)=0$, quer dizer $d_{x} F$ é sobrejetora em $x$. Portanto, existe uma vizinhança $U$ de 0 tal que

$$
d_{x} F\left(\mathbb{C}^{N}\right)=T_{F(x)} M_{m, n}, \forall x \in U
$$

Ou seja, temos que $N \geqslant m \times n$ e $F$ localmente é equivalente a uma projeção.

Considere a seguinte aplicação:

$$
\begin{array}{cccc}
\bar{F}: \mathbb{C}^{N} \times G r\left(d_{m, n}^{t}, m n\right) & \rightarrow & M_{m, n} \times G r\left(d_{m, n}^{t}, m n\right) \\
(x, T) & \mapsto & (F(x), T)
\end{array}
$$

onde $d_{m, n}^{t}=\operatorname{dim} M_{m, n}^{t}=m n-(m-t+1)(n-t+1)$.

Queremos mostrar que $\bar{F}$ é transversal a $\widehat{M}_{m, n}^{t}$. De fato, seja $x$ tal que $F(x) \in M_{m, n}^{i} \backslash M_{m, n}^{i-1}$, $x \in U$, como $F \pitchfork M_{m, n}^{i} \backslash M_{m, n}^{i-1}$ em $F(x)$ temos

$$
d_{x} F\left(\mathbb{C}^{N}\right)+T_{F(x)}\left(M_{m, n}^{i} \backslash M_{m, n}^{i-1}\right)=T_{F(x)} M_{m, n}
$$

Assim

$$
\begin{aligned}
d_{x} F\left(\mathbb{C}^{N}\right) \oplus T_{W} G r\left(d_{m, n}^{t}, m n\right)+T_{(F(x), W)} \widehat{M}_{n, m}^{t} & =M_{m, n} \oplus T_{W} G r\left(d_{m, n}^{t}, m n\right) . \\
d_{(x, W)} \bar{F}\left(\mathbb{C}^{N} \oplus T_{W} G r\left(d_{m, n}^{t}, m n\right)\right)+T_{(F(x), W)} \widehat{M}_{m, n}^{t} & =M_{m, n} \oplus T_{W} G r\left(d_{m, n}^{t}, m n\right),
\end{aligned}
$$

e portanto $\bar{F}$ é transversal a $\widehat{M}_{m, n}^{t}$ em $(x, W)$. Então $\bar{X}=\bar{F}^{-1}\left(\widehat{M}_{m, n}^{t}\right)$ é suave.

Vamos mostrar que $\bar{X} \cong \widehat{X}$. De fato, considere a seguinte aplicação 


$$
\begin{array}{cccc}
\Psi: & \bar{X} & \rightarrow & \widehat{X} \\
(x, W) & \mapsto & \left(x,\left(d_{x} F\right)^{-1}(W)\right) .
\end{array}
$$

A aplicação $\Psi$ está bem definida pois para um ponto singular $x \in X,(x, W) \in \bar{X}$, $(F(x), W) \in \widehat{M}_{m, n}^{t}$, existe uma seqüência $x_{r} \rightarrow x, F\left(x_{r}\right) \rightarrow F(x), x_{r} \in X_{r e g}=F^{-1}\left(M_{m, n}^{t} \backslash M_{m, n}^{t}\right)$ tal que $\lim T_{F\left(x_{r}\right)} M_{m, n}^{t}=W$. Como $F$ é transversal aos estratos de $M_{m, n}^{t}$, então

$$
\left(d_{x_{r}} F\right)^{-1}\left(T_{F\left(x_{r}\right)}\left(M_{m, n}^{t} \backslash M_{m, n}^{t-1}\right)=T_{x_{r}} X_{r e g} \text { e } T=\left(d_{x} F\right)^{-1}(W)\right.
$$

A restrição da projeção $\pi: M_{m, n} \times G r\left(d_{m, n}^{t}, m n\right) \rightarrow M_{m, n}$ a $\widehat{M}_{m, n}^{t}$ é a aplicação $\nu_{t}:$ $\widehat{M}_{m, n}^{t} \rightarrow M_{m, n}^{t}$.

Lema 4.8. $S e(F(x), W) \in \nu_{t}^{-1}\left(M_{m, n}^{i} \backslash M_{m, n}^{i-1}\right)$ então

$$
d_{(F(x), W)} \nu_{t}\left(T_{(F(x), W)} \widehat{M}_{m, n}^{t}\right) \supset T_{F(x)}\left(M_{m, n}^{i} \backslash M_{m, n}^{i-1}\right) .
$$

Demonstração: Como $(F(x), W) \in \widehat{M}_{m, n}^{t}$, então existe uma seqüência $x_{k} \rightarrow x$, com $x_{k} \in$ $X_{r e g}, \operatorname{assim} F\left(x_{k}\right) \in M_{m, n}^{t} \backslash M_{m, n}^{t-1}, F\left(x_{k}\right) \rightarrow F(x)$ e $T_{F\left(x_{k}\right)}\left(M_{m, n}^{t} \backslash M_{m, n}^{t-1}\right) \rightarrow W$.

Temos que a aplicação $\nu_{t}: \widehat{M}_{m, n}^{t} \backslash \nu_{t}^{-1}\left(M_{m, n}^{t-1}\right) \rightarrow M_{m, n}^{t} \backslash M_{m, n}^{t-1}$ é um difeomorfismo, se denotamos $W_{k}=T_{F\left(x_{k}\right)}\left(M_{m, n}^{t} \backslash M_{m, n}^{t-1}\right)$ então

$$
d_{\left(F\left(x_{k}\right), W_{k}\right)} \nu_{t}\left(T_{\left(F\left(x_{k}\right), W_{k}\right)} \widehat{M}_{m, n}^{t}\right)=T_{F\left(x_{k}\right)}\left(M_{m, n}^{t} \backslash M_{m, n}^{t-1}\right)
$$

Aplicando limite temos que

$$
d_{(F(x), W)} \nu_{t}\left(T_{(F(x), W)} \widehat{M}_{m, n}^{t}\right)=W
$$

Como a estratificação de $M_{m, n}^{t}$ é Whitney, então pela condição $a$ ) temos que

$$
W \supset T_{F(x)}\left(M_{m, n}^{i} \backslash M_{m, n}^{i-1}\right) .
$$

Prova do Teorema 4.6 Vamos provar que se $F$ é transversal aos limites dos espaços tangentes aos estratos, então $\bar{X}$ é suave.

Se $x \notin F^{-1}(0), F(x) \in M_{m, n}^{i} \backslash M_{m, n}^{i-1}$, então $F \pitchfork\left\{M_{m, n}^{i} \backslash M_{m, n}^{i-1}\right\}$ em $x$. Isto é

$$
d_{x} F\left(\mathbb{C}^{N}\right)+T_{F(x)}\left(M_{m, n}^{i} \backslash M_{m, n}^{i-1}\right)=M_{m, n}
$$


Então afirmamos que

$$
d_{(x, W)} \bar{F}\left(\mathbb{C}^{N} \oplus T_{W} G r\left(d_{m, n}^{t}, m n\right)\right)+T_{(F(x), W)} \widehat{M}_{m, n}^{t}=M_{m, n} \oplus T_{W} G r\left(d_{m, n}^{t}, m n\right) .
$$

De fato, pelo Lema 4.8 , como $(F(x), W) \in \nu_{t}^{-1}\left(M_{m, n}^{i} \backslash M_{m, n}^{i-1}\right)$ então

$$
d_{(F(x), W)} \nu_{t}\left(T_{(F(x), W))} M_{m, n}^{t}\right) \supset T_{F(x)}\left(M_{m, n}^{i} \backslash M_{m, n}^{i-1}\right) .
$$

O resultado segue da Equacão 4.5).

Se $x \in F^{-1}(0)$, por hipótese $F$ é transversal aos limites dos espaços tangentes aos estratos de $M_{m, n}^{t}$. Isto é, $\forall x_{k} \rightarrow x, F\left(x_{k}\right) \rightarrow 0, F\left(x_{k}\right) \in M_{m, n}^{i} \backslash M_{m, n}^{i-1}, T_{F\left(x_{k}\right)}\left(M_{m, n}^{i} \backslash M_{m, n}^{i-1}\right) \rightarrow T^{i}$, então

$$
d_{x} F\left(\mathbb{C}^{N}\right)+T^{i}=M_{m, n}
$$

Seja $(0, W) \in \nu_{t}^{-1}(0)$. Queremos mostrar que

$$
\left.d_{x} F\left(\mathbb{C}^{N}\right) \oplus T_{W} G r\left(d_{m, n}^{t}, m n\right)\right)+T_{(0, W)} \widehat{M}_{m, n}^{t}=M_{m, n} \oplus T_{W} G r\left(d_{m, n}^{t}, m n\right)
$$

É suficiente mostrar que $\forall T^{i}$ como acima

$$
d_{(0, W)} \nu_{t}\left(T_{(0, W)}\left(\widehat{M}_{m, n}^{t}\right)\right) \supset T^{i}
$$

Seja $T^{i}$ como na Equação 4.7. Para cada $k$ seja $x_{k l}$ uma seqüência em $X_{r e g}$ tal que $x_{k l} \rightarrow x_{k}$, então temos que $F\left(x_{k l}\right) \in M_{m, n}^{t} \backslash M_{m, n}^{t-1}, F\left(x_{k l}\right) \rightarrow F\left(x_{k}\right) \in M_{m, n}^{i} \backslash M_{m, n}^{i-1} \mathrm{e}$

$$
W_{k}=\lim _{F\left(x_{k l}\right) \rightarrow F\left(x_{k}\right)} T_{F\left(x_{k l}\right)}\left(M_{m, n}^{t} \backslash M_{m, n}^{t-1}\right) .
$$

Tomando a seqüência diagonal $x_{k k} \rightarrow x$, temos $F\left(x_{k k}\right) \rightarrow F(x)=0, T_{F\left(x_{k k}\right)}\left(M_{m, n}^{t} \backslash M_{m, n}^{t-1}\right) \rightarrow$ $W$. Então $(0, W) \in \nu_{t}^{-1}(0)$ e $W_{k} \rightarrow W$. Temos que $\left(F\left(x_{k}\right), W_{k}\right) \in \nu^{-1}\left(M_{m, n}^{i} \backslash M_{m, n}^{i-1}\right)$, pelo Lema 4.8 segue que

$$
d_{\left(F\left(x_{k}\right), W_{k}\right)} \nu_{t}\left(T_{\left(F\left(x_{k}\right), W_{k}\right)} \widehat{M}_{m, n}^{t}\right) \supset T_{F\left(x_{k}\right)}\left(M_{m, n}^{i} \backslash M_{m, n}^{i-1}\right)
$$

Aplicando limite, obtemos

$$
d_{(0, W)} \nu_{t}\left(T_{(0, W)} \widehat{M}_{m, n}^{t}\right) \supset W \supset T^{i}
$$

Assim $\bar{X}$ é suave. 


\subsection{Exemplos}

$\mathrm{Na}$ seqüência apresentamos alguns exemplos de transformada de Nash de EIDS. Para o cálculo usamos a definição da transformada de Nash. As matrizes nos exemplos são obtidas da tabela de A. Frühbis-Krüger e A. Neumer em [18].

Exemplo 4.9. Considere

$$
\begin{aligned}
F: \mathbb{C}^{4} & \rightarrow \\
(x, y, z, w) & \mapsto\left(\begin{array}{ccc}
w & M_{2,3} \\
w & y & x \\
z & w & y
\end{array}\right) .
\end{aligned}
$$

Então $X=V\left(w^{2}-z y, w y-z x, y^{2}-w x\right)$, tem uma singularidade isolada na origem.

A fibra $\nu^{-1}(0)$ é igual $a$,

$$
\begin{gathered}
\nu^{-1}(0)=\left\{(0, T) / T=\lim _{x_{r} \rightarrow 0}\left(d_{x_{r}} F\right)^{-1}\left(T_{F\left(x_{r}\right)} M_{2,3}^{2} \backslash M_{2,3}^{1}\right)\right\} . \\
\nu^{-1}(0)=\left\{(0, T) / T=\left(d_{x} F\right)^{-1}\left(\lim _{x_{r} \rightarrow 0} T_{F\left(x_{r}\right)} M_{2,3}^{2} \backslash M_{2,3}^{1}\right)\right\} . \\
\nu^{-1}(0)=\left\{(0, T) / T=\left(d_{x} F\right)^{-1}(W), W=\alpha\left(W_{1}, W_{2}\right), W_{1} \subset \mathbb{C}^{3},\{0\} \subset W_{2}\right\}
\end{gathered}
$$

Temos que a dimensão de $W_{1}$ é 2 e a dimensão de $W_{2}$ é $1, F(0)$ é a matriz nula, ker $F(0)=$ $\mathbb{C}^{3}$ e $\operatorname{Im} F(\{0\})=\{0\}$. Calculamos o limite da diferencial de $F$ :

$$
d_{(x, y, z, w)} F(a, b, c, d)=\left(\begin{array}{lll}
d & b & a \\
c & d & b
\end{array}\right) .
$$

Logo, como $W_{1} \subset \mathbb{C}^{3}$ é de dimensão dois, podemos considerar sem perda de generalidade $W_{1}$ gerada pelos vetores $(1,0,0) e(0,1,0) e W_{2}=\langle(1,0)\rangle$ assim:

$$
W=\alpha\left(W_{1}, W_{2}\right)=\left\{B \in M_{2,3} \mid B . W_{1} \subset W_{2}\right\}
$$

$$
\left(\begin{array}{lll}
a & b & c \\
d & e & f
\end{array}\right)\left(\begin{array}{ll}
1 & 0 \\
0 & 1 \\
0 & 0
\end{array}\right)=\left(\begin{array}{ll}
a & b \\
d & e
\end{array}\right) .
$$

Como $W_{2}=\langle(1,0)\rangle$, então $d=e=0$.

$$
\begin{aligned}
& \text { Assim } W=\left\{B=\left(\begin{array}{lll}
a & b & c \\
d & e & f
\end{array}\right) \mid d=e=0\right\} \\
& T=\left(d_{x} F\right)^{-1}(W)=\langle(a, b, c, d) \mid c=d=0\rangle . \text { Portanto }(0, T) \in \nu^{-1}(0) .
\end{aligned}
$$

Se consideramos outras bases $W_{1}, W_{2}$ que satisfazem as condições acima mencionadas temos outros elementos na fibra. 
Para os próximos exemplos calculamos os limites de planos tangentes em $x=0$.

Exemplo 4.10. Seja $F$ dada por:

$$
\begin{aligned}
F: \mathbb{C}^{4} & \rightarrow \\
(x, y, z, w) & \mapsto\left(\begin{array}{ccc}
z & M_{2,3} \\
w & z & y
\end{array}\right)
\end{aligned}
$$

Então a variedade determinantal $X$ é uma superfície dada pelos zeros das funções:

1) $z^{2}-w y=0$

2) $z y-x w=0$

3) $y^{2}-x z=0$

Podemos ver $X$ como o cone de uma curva projetiva $X^{*}$ em $\mathbb{P}^{3}$ parametrizada por

$$
\begin{array}{cccc}
\nu: & \mathbb{P}^{1} & \rightarrow & \mathbb{P}^{3} \\
(S: T) & \mapsto & \left(S^{3}: S^{2} T: S T^{2}: T^{3}\right)
\end{array}
$$

Ou seja, $X=C\left(X^{*}\right) \subset \mathbb{C}^{4}$ onde $X^{*}$ é uma curva suave isomorfa a $\mathbb{P}^{1}$.

Vamos calcular os planos limites, ou seja, os limites da sequência $T_{x_{n}} X_{\text {reg }}$ quando $x_{n}$ converge para $x$, que é equivalente pelo Lema de seleção da curva a calcular o limite de $T_{\delta(t)} X_{r e g}$ com $\delta(t)$, uma curva em $X$ convergendo para 0 , quando $t$ converge para 0 .

Considerando uma carta, quando $x \neq 0$, temos que a variedade pode se representar com as seguintes coordenadas:

$$
\left(x, y, \frac{y^{2}}{x}, \frac{y^{3}}{x^{2}}\right)
$$

Agora considerando as derivadas com relação a $x$ e $y$, temos o espaço tangente gerado por:

$$
\left\langle\left(1,0,-\frac{y^{2}}{x^{2}},-2 \frac{y^{3}}{x^{3}}\right),\left(0,1,2 \frac{y}{x}, 3 \frac{y^{2}}{x^{2}}\right)\right\rangle .
$$

Seja $\delta(t)=(x(t), y(t), z(t), w(t))$ em $X$ uma curva em $X$, tal que $\lim _{t \rightarrow 0} \delta(t)=0$. Se $x(t)=a_{0} t^{\alpha_{0}}+\cdots$ e $y(t)=b_{0} t^{\beta_{0}}+\cdots$, então $2 \alpha_{0}<3 \beta_{0}$, assim:

1. Se $\alpha_{0}<\beta_{0}$, então o plano limite é gerado por

$$
\langle(1,0,0,0),(0,1,0,0)\rangle
$$

2. Se $\alpha_{0}=\beta_{0}$, então o plano limite é gerado por

$$
\left\langle\left(1,0,-\frac{b_{0}^{2}}{a_{0}^{2}},-2 \frac{b_{0}^{3}}{a_{0}^{3}}\right),\left(0,1,2 \frac{b_{0}}{a_{0}}, 3 \frac{b_{0}^{2}}{a_{0}^{2}}\right)\right\rangle .
$$


Quando $x \rightarrow 0$, temos o seguinte limite de planos tangentes:

$$
\langle(0,0,1,0),(0,0,0,1)\rangle \text {. }
$$

Com estes resultados obtemos $\nu^{-1}(0,0,0,0)=X^{*}$ e $X=C\left(X^{*}\right)$.

Exemplo 4.11. Seja $F$ dada pela seguinte matriz, para $k \geqslant 2$

$$
\begin{aligned}
F: \mathbb{C}^{4} & \rightarrow \\
(x, y, z, w) & \mapsto\left(\begin{array}{ccc}
z & M_{2,3} \\
w & z & y^{k}
\end{array}\right)
\end{aligned}
$$

A superfície $X=F^{-1}\left(M_{2,3}^{2}\right)$ é definida pelos zeros das seguintes funções

1. $z^{2}-w y=0$

2. $z y^{k}-w x=0$

3. $y^{k+1}-z x=0$

Considerando uma carta, quando $x \neq 0$, temos que a variedade pode se representar com as seguintes coordenadas:

$$
\left(x, y, \frac{y^{k+1}}{x}, \frac{y^{2 k+1}}{x^{2}}\right) .
$$

Agora considerando as derivadas com relação a $x$ e $y$, temos o espaço tangente gerado por:

$$
\left\langle\left(1,0,-\frac{y^{k+1}}{x^{2}},-2 \frac{y^{2 k+1}}{x^{3}}\right),\left(0,1,(k+1) \frac{y^{k}}{x},(2 k+1) \frac{y^{2 k}}{x^{2}}\right)\right\rangle .
$$

Tirando os denominadores temos que o espaço tangente é gerado por:

$$
\left\langle\left(x^{3}, 0,-x y^{k+1},-2 y^{2 k+1}\right),\left(0, x^{2},(k+1) x y^{k},(2 k+1) y^{2 k}\right)\right\rangle .
$$

Dada uma curva $\delta(t)=(x(t), y(t), z(t), w(t))$ em $X$, tal que $\lim _{t \rightarrow 0} \delta(t)=0$, se:

$$
\begin{gathered}
x(t)=a_{0} t^{\alpha_{0}}+\cdots \text { e } y(t)=b_{0} t^{\beta_{0}}+\cdots, \text { então } 2 \alpha_{0}<(2 k+1) \beta_{0}, \text { assim: } \\
\left\langle\left(a_{0}^{3} t^{3 \alpha_{0}}+\cdots, 0,-a_{0} b^{k+1} t^{\alpha_{0}+(k+1) \beta_{0}}+\cdots,-2 b_{0}^{2 k+1} t^{(2 k+1) \beta_{0}}+\cdots\right),\left(0, a_{0}^{2} t^{2 \alpha_{0}}+\cdots,(k+\right.\right. \\
\text { 1) } \left.\left.a_{0} b_{0}^{k} t^{\alpha_{0}+k \beta_{0}}+\cdots,(2 k+1) b_{0}^{2 k} t^{2 k \beta_{0}}+\cdots\right)\right\rangle .
\end{gathered}
$$

Fazendo por casos:

1. Se $\alpha_{0} \leqslant \beta_{0}$, então o plano limite é gerado por

$$
\langle(1,0,0,0),(0,1,0,0)\rangle \text {. }
$$


2. Se $\beta_{0}<\alpha_{0}<\frac{(k+1) \beta_{0}}{2}$, então $3 \alpha_{0}<\alpha_{0}+(k+1) \beta_{0}<(2 k+1) \beta_{0}$ e $2 \alpha_{0}<\alpha_{0}+k \beta_{0}<$ $2 k \beta_{0}, \operatorname{logo}$, o plano limite é gerado por

$$
\langle(1,0,0,0),(0,1,0,0)\rangle
$$

3. Se $2 \alpha_{0}=(k+1) \beta_{0}$, então o plano tangente é gerado por:

$$
\left\langle\left(a_{0}^{2}, 0,-b_{0}^{k+1}, 0\right),(0,1,0,0)\right\rangle .
$$

4. Se $\frac{(k+1) \beta_{0}}{2}<\alpha_{0}<k \beta_{0}$, então $\alpha_{0}+(k+1) \beta_{0}<(2 k+1) \beta_{0}<3 \alpha_{0}$ e $2 \alpha_{0}<\alpha_{0}+k \beta_{0}<$ $2 k \beta_{0}, \log$ o o plano é gerado por

$$
\langle(0,0,1,0),(0,1,0,0)\rangle \text {. }
$$

5. $\alpha_{0}=k \beta_{0}$, então o plano limite é gerado por

$$
\left\langle\left(0,0, a_{0}, 2 b_{0}^{k}\right),\left(0, a_{0}^{2},(k+1) a_{0} b_{0}^{k},(2 k+1) b_{0}^{2 k}\right)\right\rangle .
$$

6. $k \beta_{0}<\alpha_{0}<\frac{(2 k+1) \beta_{0}}{2}$, então os limites de vetores tangentes são paralelos

$$
\langle(0,0,0,1)\rangle \text {. }
$$

7. Se $x=0$, temos o seguinte limite de planos tangentes

$$
\langle(0,0,1,0),(0,0,0,1)\rangle \text {. }
$$

Podemos ver que o conjunto de limites de espaços tangentes na origem se dá através do cone tangente unido com um feixe de planos que são gerados por $(0,1,0,0)$ e $\left(a_{0}^{2}, 0,-b_{0}^{k+1}, 0\right)$, concluindo que a transformada de Nash não é suave. 


\section{Espaço Conormal de Variedades Determinantais}

O objetivo deste capítulo é estudar os limites de hiperplanos tangentes de uma variedade determinantal. Definiremos hiperplano geral e fortemente geral sobre uma IDS ou EIDS respetivamente. Estas duas definições são equivalentes no caso de singularidade isolada. As seções gerais definidas pela interseção de um hiperplano geral (fortemente geral) com a variedade determinantal determina uma variedade determinantal com singularidade isolada ou uma EIDS respectivamente. Jawad Snoussi estudou o limite de hiperplanos tangentes de superfícies normais em [38], dando algumas caracterizações das seções gerais em termos da minimalidade do número de Milnor da curva. Como toda superfície determinantal com singularidade isolada é normal o resultado se aplica para superfícies determinantais em $\mathbb{C}^{4}$. Neste capítulo estudamos as seções gerais de uma 3 variedade determinantal em $\mathbb{C}^{5}$. Tais seções são superfícies determinantais, e portanto seu número de Milnor está bem definido. No Teorema 5.21 provamos que o número de Milnor de uma seção geral é mínimo.

Para o caso de EIDS, podemos considerar as seções gerais, dadas pela interseção da variedade $X$ com um plano linear $P$, de codimensão $d-2$ tal que $\operatorname{dim} X \cap P=2$. Neste caso, obtemos um resultado análogo ao de Lê Dung Trang en [26]. No Teorema 5.24 provamos que se $H$ e $H^{\prime}$ são dois hiperplanos fortemente gerais então eles contêm $P$ e $P^{\prime}$ espaços lineares de codimensão $d-2$, tais que os respectivos números de Milnor das seções gerais, que são superfícies determinantais, são iguais.

\subsection{Limite de Hiperplanos de Espaços Tangentes}

Seja $(X, 0) \subset\left(\mathbb{C}^{N}, 0\right)$ um germe de variedade analítica $d$-dimensional reduzida. Denotamos por $X$ um representante suficientemente pequeno do germe $(X, 0)$ e por $X_{0}=X_{\text {reg }}$ a parte regular de $X$ (para não fazer confusão com a notação, note que se $X$ tem uma estratificação de 
Whitney $V_{0}=\{0\}$ é o estrato de $\left.X\right)$.

Consideremos o subconjunto $C_{0}\left(X_{0}, \mathbb{C}^{N}\right)$ de $X_{0} \times \check{\mathbb{C}}^{N}$, definido assim:

$C_{0}\left(X_{0}, \mathbb{C}^{N}\right)=\left\{(x, \xi) / x \in X_{0}\right.$ e $\xi: \mathbb{C}^{N} \rightarrow \mathbb{C}$, é uma forma linear com $\left.\xi\left(T_{x} X_{0}\right)=0\right\}$

Definição 5.1. O fecho da projetivização com relação ao segundo fator de $C_{0}\left(X_{0}, \mathbb{C}^{N}\right)$ em $X \times \check{\mathbb{P}}^{N-1}$, é chamado espaço conormal associado $X$ em $\mathbb{C}^{N}$, denotado por $C\left(X, \mathbb{C}^{N}\right)$ ou simplesmente $C(X)$. Denotaremos por $\eta: C\left(X, \mathbb{C}^{N}\right) \rightarrow X$ o morfismo induzido pela primeira projeção, chamado morfismo conormal.

Observação 5.2. Para um germe $(X, 0)$ de variedade analítica as seguintes propriedades se verificam, ver [39]:

1. O conjunto $C(X)$ é um subespaço analítico de $X \times \check{\mathbb{P}}^{N-1}$ de dimensão $N-1$.

2. Se $x$ é um ponto não singular do espaço $X$, então $\eta^{-1}(x)$ corresponde ao conjunto de hiperplanos de $\mathbb{C}^{N}$ tangentes ao espaço $X$ em $x$ e $\operatorname{dim} \eta^{-1}(x)=N-d-1$.

3. Um hiperplano $H \in \eta^{-1}(0) \subset \mathbb{C}^{N}$ é um limite de hiperplanos tangentes ao espaço $X$ em 0 , se existe uma sequência de pontos não singulares $\left(x_{n}\right)$ de $X$ que converge a 0 e uma sequência de hiperplanos $H_{n} \supset T_{x_{n}} X_{\text {reg }}$ de $\mathbb{C}^{N}$, tais que $H_{n}$ converge à $H$ em $\check{\mathbb{P}}^{N-1}$.

4. A fibra $\eta^{-1}(0)$ em um ponto singular tem dimensão $N-d+k-1$, onde $k=\operatorname{dim} \nu^{-1}(0)$ $(\nu: \widehat{X} \rightarrow X$ é a aplicação definida pela transformação de Nash, ver Definição 1.9). Como $k \leqslant d-1$, temos que $\operatorname{dim} \eta^{-1}(0) \leqslant N-2$.

Denotemos por $C_{0}(X)=\eta^{-1}(0)$ o conjunto de todos os limites de hiperplanos tangentes a $X$ em 0 .

Denotemos por $\phi:(\mathbb{C}, \mathbb{C}-0,0) \rightarrow\left(X, X_{0}, 0\right)$ o germe de curva analítica tal que $\phi(0)=0$ e $\phi(\mathbb{C}-\{0\}) \subset X_{0}$

Lema 5.3. [21] Seja $F:\left(\mathbb{C}^{N}, 0\right) \rightarrow\left(\mathbb{C}^{p}, 0\right)$, com $X=F^{-1}(0)$. Um hiperplano $H$ pertence a $C_{0}(X)$ se somente se existe um germe de curva analítica $\phi:(\mathbb{C}, \mathbb{C}-0,0) \rightarrow\left(X, X_{0}, 0\right)$ e uma aplicação $\psi:(\mathbb{C}, 0) \rightarrow \operatorname{Hom}\left(\mathbb{C}^{p}, \mathbb{C}\right)$ tais que para algum $k$,

$$
\left(a_{1}, a_{2}, \cdots, a_{N}\right)=\lim _{t \rightarrow 0} \frac{1}{t^{k}} \psi(t)(D F(\phi(t)))
$$

onde $\sum_{i}^{N} a_{i} x_{i}=0$ é a equação de $H$. 
Se $H$ é um hiperplano em $\mathbb{C}^{N}$, denotemos $J M(F)_{H}$ o submódulos de $J M(F)$ gerado por $\partial F / \partial v, \forall v \in H$.

O seguinte resultado é uma caracterização do conjunto de limites de hiperplanos tangentes. Apresentamos a prova com mais detalhes.

Teorema 5.4. [21] 13] Seja $H$ um hiperplano em $\mathbb{C}^{N}$, temos que $H \in C_{0}(X)$ se somente se, $J M(F)_{H}$ não é redução de $J M(F)$.

Demonstração: Pelo lema 5.3, temos que $H=\left\{\left(x_{1}, \cdots, x_{N}\right) / \sum_{i=1}^{N} a_{i} x_{i}=0\right\} \in C(X)$ se existem $\phi:(\mathbb{C}, \mathbb{C}-\{0\}, 0) \rightarrow\left(X, X_{0}, 0\right), \psi:(\mathbb{C}, 0) \rightarrow \operatorname{Hom}\left(\mathbb{C}^{p}, \mathbb{C}\right)$ e $k>0$, tais que

$$
\left(a_{1}, \cdots, a_{N}\right)=\lim _{t \rightarrow 0} \frac{1}{t^{k}} \psi(t)(D F(\phi(t))) .
$$

Por outro lado temos que $I_{\psi} \phi^{*}\left(J M(F)_{H}\right)=\mathcal{O}_{1}\{\psi(t)(D F(\phi(t) . v)) / v \in H\}$ ( $\phi^{*}$ é definido no Capítulo 1, Seção 1.5), como $I_{\psi}\left(\phi^{*}(N)\right) \subset \mathcal{O}_{1}$, então $I_{\psi} \phi^{*}\left(J M(F)_{H}\right)=\mathcal{O}_{1}\left\{t^{q}\right\}$, para algum $q \in \mathbb{N}$. Pela equação 5.1), temos que: $I_{\psi} \phi^{*}\left(J M(F)_{H}\right)=\mathcal{O}_{1}\left\{t^{q}\right\}$, para $q>k$.

Da equação [5.1), temos que $\lim _{t \rightarrow 0} \frac{1}{t^{k}} \psi(t)(D F(\phi(t))) . u \neq 0$ para qualquer $u \in \mathbb{C}^{N}-H$. Logo $\psi(t)(D F(\phi(t))) . u \notin I_{\psi} \phi^{*}\left(J M(\vec{F})_{H}\right)$, segue da proposição 1.23 , que $D F(x) u \notin J M(F)_{H}$.

Reciprocamente, se $\overline{J M(F)_{H}} \varsubsetneqq \overline{J M(F)}$, existe $u \in \mathbb{C}^{N}-H$ tal que $D(F) . u \notin J M(F)_{H}$, logo pela proposição 1.23 , existem $\phi:(\mathbb{C}, \mathbb{C}-0,0) \rightarrow\left(X, X_{0}, 0\right), \psi:(\mathbb{C}, 0) \rightarrow \operatorname{Hom}\left(\mathbb{C}^{p}, \mathbb{C}\right)$ tal que $\psi(t)(D F(\phi(t))) . u \notin I_{\psi} \phi^{*}\left(J M(F)_{H}\right)$. Como $I_{\psi} \phi^{*}\left(J M(F)_{H}\right) \subseteq I_{\psi} \phi^{*}(J M(F)) \subseteq \mathcal{O}_{1}$, existem $k, q \in \mathbb{N}$ tais que $I_{\psi} \phi^{*}\left(J M(F)_{H}\right)=\mathcal{O}_{1}\left\{t^{q}\right\}, I_{\psi} \phi^{*}(J M(F))=\mathcal{O}_{1}\left\{t^{k}\right\}$, com $0 \leqslant k \leqslant q$. Como $\psi(t)(D F(\phi(t))) . u \notin I_{\psi} \phi^{*}\left(J M(F)_{H}\right)$, então $0 \leqslant k<q$. Como $I_{\psi} \phi^{*}(J M(F))=$ $\mathcal{O}_{1}\left\{t^{k}\right\}$, segue que

$$
\lim _{t \rightarrow 0} \frac{1}{t^{k}} \psi(t)(D F(\phi(t)))=\left(a_{1}, a_{2}, \cdots, a_{N}\right) \neq 0
$$

e pelo fato que $I_{\psi} \phi^{*}\left(J M(F)_{H}\right)=\mathcal{O}_{1}\left\{t^{q}\right\}$ e $0 \leqslant k<q$, segue que

$$
\lim _{t \rightarrow 0} \frac{1}{t^{k}} \psi(t)(D F(\phi(t)))=0
$$

portanto $\sum_{i=1}^{N} a_{i} x_{i}=0, \forall x \in H$. Assim $H=\left\{\left(x_{1}, \cdots, x_{N}\right) / \sum_{i=1}^{N} a_{i} x_{i}=0\right\}$ e pelo lema 5.3 segue que $H \in C_{0}(X)$.

No artigo [38], o autor define hiperplano geral se ele não é limite de espaços tangentes. Aqui daremos uma definição equivalente.

Definição 5.5. Dizemos que um hiperplano $H$ em $\mathbb{C}^{N}$, dado pelo núcleo de uma aplicação linear $p: \mathbb{C}^{N} \rightarrow \mathbb{C}$ é geral com relação a $X$ em 0 se $H$ é transversal a $T, \forall T \in \nu^{-1}(0)$ (limite de espaços tangentes). 
Proposição 5.6. Seja $X$ uma variedade analítica em $\mathbb{C}^{N}$. Existe um conjunto Zariski aberto não vazio $\Omega \subset \mathbb{P}^{N-1}$ tal que $\forall H \in \Omega$, H é geral a $X$ em 0 .

Demonstração: A proposição segue da observação 5.2 $\operatorname{com} \Omega=\mathbb{P}^{N-1} \backslash \eta^{-1}(0)$, pois $\eta^{-1}(0)$ é um conjunto analítico próprio.

Na seguinte proposição mostraremos que a Definição 2.2 (pag. 64) dada no artigo [38] e a definição 5.5 são equivalentes.

Proposição 5.7. Um hiperplano $H$ é limite de hiperplanos tangentes a X em 0 se, somente se H não é geral.

Demonstração: Suponha que $H$ é um limite de hiperplanos tangentes, então existe uma sequência $\left(x_{n}\right)$ de pontos na parte regular de $X$, tal que $T=\lim T_{x_{n}} X_{\text {reg }} \subset H=p^{-1}(0)$. Logo $\operatorname{ker}(p)$ não é transversal à T.

Reciprocamente, suponha que $H$ não é geral, então, existe uma sequência $\left(x_{n}\right) \in X_{\text {reg }}$ com $T=\lim T_{x_{n} \rightarrow 0} X_{\text {reg }}$, tal que $H$ não é transversal à $T$. Isto quer dizer que $T+H \neq \mathbb{C}^{N}$. Como $\operatorname{dim} H=N-1$ e $\operatorname{dim} T=2$, então $T \subseteq H$, ou seja, $p(T)=0$, assim $H=p^{-1}(0)$ é limite de hiperplanos tangentes.

Na seguinte proposição vamos mostrar que a condição " $H$ é geral" vale para todos os pontos regulares em uma vizinhança de 0 .

Proposição 5.8. Se H é geral em 0 então existe um aberto $U$ contendo 0 , tal que $H$ é transversal a $X$ em $y, \forall y \in U \cap X_{\text {reg }}$

Demonstração: Suponha que a condição não seja verdadeira. Pelo lema da seleção da curva, existe uma curva $\alpha$ em $X$, tal que $\alpha(t) \neq 0$ quando $t \neq 0$ e $\alpha(0)=0$, tal que $T_{\alpha(t)} X$ não é transversal à $H, \forall t \neq 0$. Isto implica que $T_{\alpha(t)} X \subset H, \forall t \neq 0$. Logo $T=\lim _{t \rightarrow 0} T_{\alpha(t)} \subset H$, o que contradiz a hipótese.

Proposição 5.9. Seja $X \subset \mathbb{C}^{N}$ uma variedade determinantal d-dimensional com singularidade isolada na origem e $H$ um hiperplano geral com relação a $X$, então $X \cap H \subset \mathbb{C}^{N-1}$ é uma variedade determinantal $d-1$ dimensional com singularidade isolada na origem.

Demonstração: Como $H$ é transversal à $T$, então $\operatorname{dim}(X \cap H)=d-1$. Assim, suponha sem perda de generalidade que $x_{1}=0$ é a equação de $H$ e $X$ é definida pela função $F: \mathbb{C}^{N} \rightarrow$ $M_{m, n}$, então $X \cap H$ é definida pela função $F^{\prime}\left(x_{2}, \cdots, x_{N}\right)=F\left(0, x_{2}, \cdots, x_{N}\right)$. Portanto $X \cap H$ é uma variedade determinantal $d-1$ dimensional em $\mathbb{C}^{N-1}$.

Segue da proposição 5.8 que $X \cap H$ tem singularidade isolada na origem. 


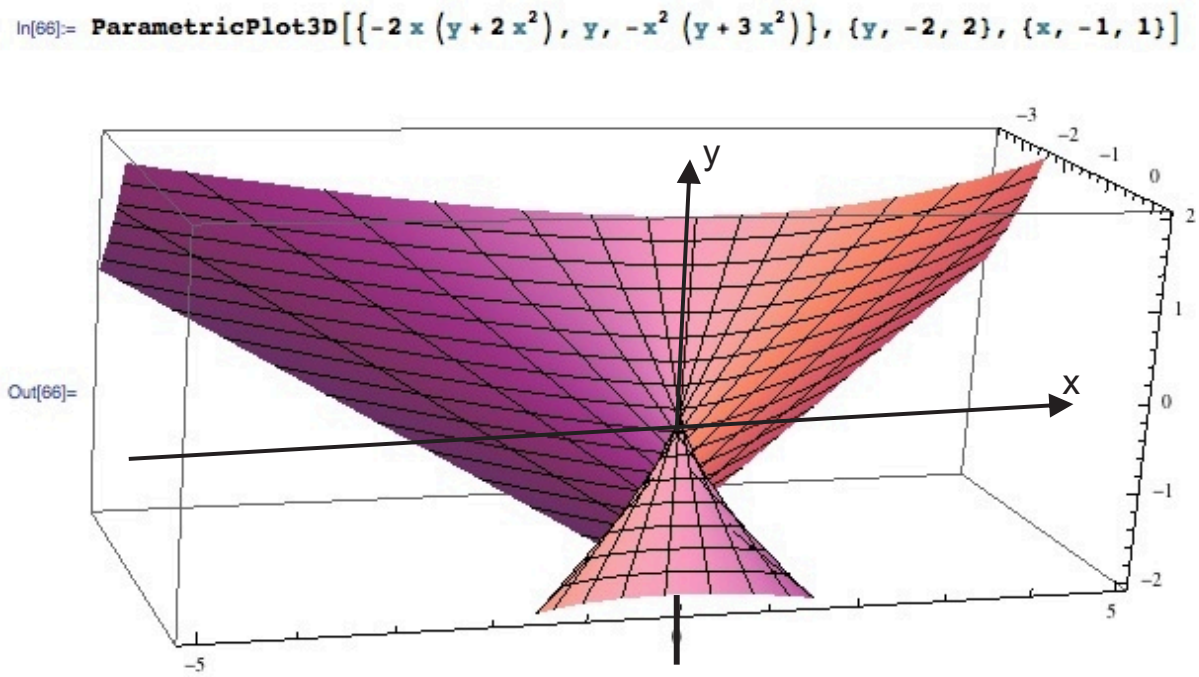

Figura 5.1:

Na sequência daremos um exemplo no qual um hiperplano $H$ não é limite de hiperplanos tangentes, mas ele não é transversal aos limites de espaços tangentes aos estratos do conjunto singular da variedade com singularidade não isolada.

Exemplo 5.10. Considere $X$ a superfície rabo de andorinha, dada na figura 5.1. X é a superficie em $\mathbb{C}^{3}$ definida pelos zeros de

$$
256 Z^{3}-27 X^{4}-128 Z^{2} Y^{2}+144 Z X^{2} Y+16 Z Y^{4}-4 X^{2} Y^{3}=0 .
$$

Ela pode também ser definida pela parametrização.

$$
\begin{aligned}
\mathbb{C}^{2} & \rightarrow \mathbb{C}^{3} \\
(x, y) & \mapsto\left(-4 x^{3}-2 x y, y,-3 x^{4}-x^{2} y\right)
\end{aligned}
$$

$O$ conjunto dos hiperplanos tangentes a $X$ em zero é o hiperplano $Z=0$ (ver por exemplo [30]).

Temos que todo hiperplano diferente do hiperplano $Z=0$ é geral, em particular o plano $H=\{(X, Y, Z): X=0\}$ é geral, mas $H$ não é transversal aos limites de retas tangentes aos estratos de dimensão 1 .

Este exemplo motiva a seguinte definição.

Definição 5.11. Seja $X$ uma variedade analítica complexa d-dimensional, e seja $\left\{V_{i}\right\}$ uma estratificação de X. Dizemos que um hiperplano $H$ é fortemente geral a X em 0 , se existe uma 
vizinhança $U$ de 0 tal que para todo estrato $V_{\lambda}$ de $X$, com $0 \in \bar{V}_{\lambda}$, temos que $H \pitchfork V_{\lambda}$ em $x, \forall$ $x \in U \backslash\{0\}$.

Se a variedade tem singularidade isolada, então as duas condições das definições (geral e fortemente geral) coincidem.

Teorema 5.12. Seja $(X, 0) \subset\left(\mathbb{C}^{N}, 0\right)$ uma EIDS, de tipo $(n, m, t)$. Se $H \subset \mathbb{C}^{N}$ é um hiperplano fortemente geral a $X$ na origem, então $X \cap H$ é uma EIDS em $\mathbb{C}^{N-1}$ de dimensão $d-1$ e do mesmo tipo.

Demonstração: Seja $F: \mathbb{C}^{N} \rightarrow M_{m, n}$ a função que define $X=F^{-1}\left(M_{m, n}^{t}\right)$. Como $X$ é uma EIDS, então $F$ é transversal aos estratos de $M_{m, n}^{t}$ em $U \backslash\{0\}$, onde $U$ é uma vizinhança da origem. Pela hipótese temos que $H$ é transversal aos estratos de $X$ fora da origem, ou seja a aplicação inclusão $i: \mathbb{C}^{N-1} \rightarrow \mathbb{C}^{N}$ é transversal aos estratos de $X$ fora da origem. Então $F \circ i: \mathbb{C}^{N-1} \rightarrow M_{m, n}$ é transversal a todos os estratos de $M_{m, n}^{t}$ fora da origem, assim $X \cap H=F^{-1}\left(M_{m, n}^{t}\right) \subset \mathbb{C}^{N-1}$ é uma EIDS do mesmo tipo do que $X$ e $\operatorname{dim}(X \cap H)=d-1$ em $\mathbb{C}^{N-1}$.

\subsection{Minimalidade do Número de Milnor}

O objetivo desta seção é provar um resultado de minimalidade análogo ao obtido por Jawad Snoussi [38], para variedades determinantais com singularidade isolada.

Os primeiros resultados sobre minimalidade do número de Milnor de uma seção genérica foram obtidos por J.-P. G. Henry e D. T. Lê para hipersuperficies em $\mathbb{C}^{N}$ em 1975, [25]. Esses resultados foram estendidos ao caso de interseções completas por Terence Gaffney, em [21]. Os dois trabalhos usam fecho integral de ideais e módulos para provar os resultados.

\subsubsection{Hipersuperfícies e Interseções Completas em $\mathbb{C}^{N}$}

Seja $X \subset U \subset \mathbb{C}^{N}$ uma hipersuperfície analítica definida em um aberto $U$ em $\mathbb{C}^{N}$ pela função $f=0$. Suponha que $X$ tem singularidade isolada na origem.

Lema 5.13. [25] Existe um conjunto Zariski aberto $\Omega$, do espaço projetivo de hiperplanos afins de $\mathbb{C}^{N}$ passando pela origem tal que $H \in \Omega$ se e somente se $(X \cap H)$ tem uma singularidade isolada na origem e o número de Milnor $\mu(X \cap H, 0)$ de $X \cap H$ na origem é mínimo entre os números de Milnor na origem de seções hiperplanas $X \cap L$, tendo singularidade isolada na origem.

Se $H \in \Omega$, então $H$ é chamado plano geral de $X$ na origem. 
Teorema 5.14. [25] Um hiperplano $H \subset \mathbb{C}^{N}$ não é limite de hiperplanos tangentes em $X$ se e somente se $\mu(X \cap H)$ é mínimo.

Demonstração: Suponha sem perda de generalidade que $H$ é definido por $x_{0}=0$

Temos a fórmula de tipo Lê-Greuel:

$$
\operatorname{dim}_{\mathbb{C}} \frac{\mathcal{O}_{\mathbb{C}^{N}, 0}}{\left\langle f, \frac{\partial f}{\partial x_{1}}, \ldots, \frac{\partial f}{\partial x_{N}}\right\rangle}=\mu(X)+\mu(X \cap H) .
$$

Então

$$
\operatorname{dim}_{\mathbb{C}} \frac{\mathcal{O}_{\mathbb{C}^{N}, 0}}{\left\langle f, \frac{\partial f}{\partial x_{1}}, \ldots, \frac{\partial f}{\partial x_{N}}\right\rangle}=e\left(\left\langle\frac{\partial f}{\partial x_{1}}, \ldots, \frac{\partial f}{\partial x_{N}}\right\rangle, \mathcal{O}_{X, x}\right),
$$

onde $e\left(I, \mathcal{O}_{X, x}\right)$ é a multiplicidade do ideal $I$ sobre $\mathcal{O}_{X, x}$.

Indicamos por $I=\left\langle\frac{\partial f}{\partial x_{1}}, \ldots, \frac{\partial f}{\partial x_{N}}\right\rangle$ e $J=\left\langle\frac{\partial f}{\partial x_{0}}, \ldots, \frac{\partial f}{\partial x_{N}}\right\rangle$ os ideais do anel $\mathcal{O}_{X, x}$, podemos usar o seguinte resultado, ver [37].

Lema 5.15. Se $I \subseteq J$ então $e\left(I, \mathcal{O}_{X, x}\right)=e\left(J, \mathcal{O}_{X, x}\right)$ se e somente se $\bar{I}=\bar{J}$.

Continuando com a prova, como $\left\langle\frac{\partial f}{\partial x_{1}}, \ldots, \frac{\partial f}{\partial x_{N}}\right\rangle \subseteq\left\langle\frac{\partial f}{\partial x_{0}}, \ldots, \frac{\partial f}{\partial x_{N}}\right\rangle$ então temos a desigualdade

$$
e\left(\left\langle\frac{\partial f}{\partial x_{0}}, \ldots, \frac{\partial f}{\partial x_{N}}\right\rangle, \mathcal{O}_{X, x}\right) \leqslant e\left(\left\langle\frac{\partial f}{\partial x_{1}}, \ldots, \frac{\partial f}{\partial x_{N}}\right\rangle, \mathcal{O}_{X, x}\right) .
$$

Logo $e\left(\left\langle\frac{\partial f}{\partial x_{0}}, \ldots, \frac{\partial f}{\partial x_{N}}\right\rangle, \mathcal{O}_{X, x}\right)=e\left(\left\langle\frac{\partial f}{\partial x_{1}}, \ldots, \frac{\partial f}{\partial x_{N}}\right\rangle, \mathcal{O}_{X, x}\right)$ se e somente se

$$
\overline{\left\langle\frac{\partial f}{\partial x_{0}}, \ldots, \frac{\partial f}{\partial x_{N}}\right\rangle}=\overline{\left\langle\frac{\partial f}{\partial x_{1}}, \ldots, \frac{\partial f}{\partial x_{N}}\right\rangle}
$$

O resultado segue de [25, Lema 2.5].

\subsubsection{Variedades Determinantais com Singularidade Isolada na Origem}

O seguinte resultado foi provado por Jawad Snoussi [38] para o caso de superfícies normais. Este lema é um dos resultados chaves para obter os resultados nesse artigo.

Seja $X \subset \mathbb{C}^{N}$ uma superfície, considere $\pi: \mathbb{C}^{N} \rightarrow \mathbb{C}^{2}$ uma aplicação linear genérica, denotemos por $\Delta_{L}=\overline{\pi\left(P_{1}(X), \pi\right)}$ o discriminante da aplicação $\pi$, onde $P_{1}(X)$ é o conjunto polar $P_{1}(X)=\overline{\left.\Sigma \pi\right|_{X_{\text {reg }}}}$

Lema 5.16. [38 Um hiperplano $H$ em $\mathbb{C}^{N}$ é geral se e somente se existe um $(N-2)$ plano $L \subset H$ tal que $L \cap\left|C_{X, 0}\right|=\{0\}$ e $\pi_{L}(H \cap X)$ não é tangente ao cone tangente $C_{\Delta_{L}, 0}$, onde $\pi: \mathbb{C}^{N} \rightarrow \mathbb{C}^{2}$ com $L=\operatorname{ker}(\pi)$. 
Para dimensões maiores, a seguinte proposição é demonstrada em [26].

Proposição 5.17. [26] Sejam $X \subset \mathbb{C}^{N}$ uma variedade analítica complexa e $L$ um subespaço afim em $\mathbb{C}^{N}$ de codimensão igual a dimensão de $C_{X, x}$ em $\mathbb{C}^{N}, x \in L$ e $L \cap C_{X, x}=\{x\}$. Considere a projeção $\pi: \mathbb{C}^{N} \rightarrow \mathbb{C}^{\operatorname{dim} X}$ tal que $\operatorname{ker} \pi=L$. Considere $\Delta_{L}$ o discriminante de $\pi$ restrito a $X$. Seja $H_{0}$ um hiperplano de $\mathbb{C}^{\operatorname{dim} X}$ que não é limite de hiperplanos tangentes do discriminante $\Delta_{L}$. Então, o hiperplano $H$ dado pela imagem inversa $H=\pi^{-1}\left(H_{0}\right)$ não é limite de hiperplanos tangentes a $X$.

O seguinte resultado é uma consequiência simples da fórmula de Lê- Greuel mas é um resultado útil.

Proposição 5.18. Seja $(X, 0) \subset \mathbb{C}^{N}$ uma IDS de dimensão $d, N<(m-t+2)(n-t+2)$. Sejam $H$ e $H^{\prime}$ hiperplanos em $\mathbb{C}^{N}$ tais que $X \cap H$ e $X \cap H^{\prime}$ são EIDS então $\nu(X \cap H, 0) \leqslant \nu\left(X \cap H^{\prime}, 0\right)$ se, e somente se $m_{d}(X, p) \leqslant m_{d}\left(X, p^{\prime}\right)$.

Demonstração: Segue diretamente do Teorema 3.15 .

$\mathrm{Na}$ sequência temos o seguinte resultado para o caso de superfícies determinantais, este resultado é um caso particular do resultado no trabalho [38], já que toda variedade determinantal é normal. A nossa prova da condição necessária usa argumentos da teoria de variedades determinantais, mas a prova recíproca é a mesma prova apresentada por Jawad Snoussi em [38].

Teorema 5.19. Sejam $X \subset \mathbb{C}^{N}$ uma superfície determinantal e $H \subset \mathbb{C}^{N}$ um hiperplano em $\mathbb{C}^{N}$. Temos que $H=p^{-1}(0)$ é geral a $X$ em 0 , se e somente se, $m_{d}(X, p)$ é mínimo.

Demonstração: Suponha que $p$ não é geral e seja $p_{t}$ uma família a 1-parâmetro de projeções tal que para $t \neq 0, p_{t}$ é geral. Podemos assumir que $X \cap H_{t}=X \cap p_{t}^{-1}(0)$ é família plana de curvas reduzidas. Pela semicontinuidade do número de Milnor $\mu$ de curvas reduzidas, então

$$
\mu\left(X \cap H_{t}\right)<\mu(X \cap H), t \neq 0
$$

Da proposição 5.18, segue que $m_{d}\left(X, p_{t}\right)<m_{d}(X, p)$, assim $m_{d}(X, p)$ não é mínimo.

Reciprocamente como em [38], suponha que $p$ é geral e $m_{d}(X, p)$ não é mínimo. pela Proposição 5.18, $\mu(X \cap H)$ não é mínimo. Por [7, Teorema 6.1.7], existe um aberto Zariski $\Omega$ do conjunto de hiperplanos em $\mathbb{C}^{N}$ tal que para todo $H^{\prime} \in \Omega$ temos que $H^{\prime} \cap X$ é uma curva reduzida e $\mu\left(H^{\prime} \cap X\right)<\mu(H \cap X)$. O conjunto $W=\left\{H \cap H^{\prime} / H \in \Omega\right\}$ é um conjunto Zariski aberto de hiperplanos de $H$. Como $H$ não contém nenhuma componente irredutível de $\left|C_{X}\right|$, o conjunto

$$
W^{\prime}=\left\{L / L \text { não contém nenhuma geratriz em comum com o cone }\left|C_{X}\right|\right\}
$$


de hiperplanos de $H$ é também aberto e denso. Então existe um hiperplano $H^{\prime} \subset \mathbb{C}^{N}$ tal que $H^{\prime} \cap X$ é uma curva reduzida e $\mu\left(H^{\prime} \cap X\right)<\mu(H \cap X)$. Seja $L=H \cap H^{\prime}, L$ é um $(N-2)$ plano de $\mathbb{C}^{N}$, então $L \cap\left|C_{X}\right|=\emptyset$, então temos:

$$
\begin{gathered}
\left(\Delta_{L} \cdot \pi_{L}(H \cap X)\right)_{0}=\mu(X \cap H)+\operatorname{deg}_{0}\left(\pi_{L}\right)-1 \\
\left(\Delta_{L} \cdot \pi_{L}\left(H^{\prime} \cap X\right)\right)_{0}=\mu\left(X \cap H^{\prime}\right)+\operatorname{deg}_{0}\left(\pi_{L}\right)-1,
\end{gathered}
$$

Denotamos por $\left(\Delta_{L} \cdot \pi_{L}\left(H^{\prime} \cap X\right)\right)_{0}$ a multiplicidade de interseção de curvas planas. Como $\mu\left(H^{\prime} \cap X\right)<\mu(H \cap X)$, então temos que $\left(\Delta_{L} \cdot \pi_{L}(H \cap X)\right)_{0}>\left(\Delta_{L} \cdot \pi_{L}\left(H^{\prime} \cap X\right)\right)_{0}$, logo por [19, pag. 40] $\pi_{L}(X \cap H)$ é tangente ao cone tangente de $\Delta_{L}$. Pelo Lema 5.16 temos que $H$ é um limite de hiperplanos tangentes.

Proposição 5.20. [28. Corolário 2.3.2.1] Sejam c um inteiro e E um subespaço vetorial de $\mathbb{C}^{N}$ de codimensão c que é a interseção de c hiperplanos $H_{1}, H_{2}, \ldots, H_{c}$ tais que $H_{i}$ não é tangente à $\left|X \cap H_{1} \cap H_{2} \cap \cdots \cap H_{i-1}\right|$, para $1 \leqslant i \leqslant c$. Então a multiplicidade em $x$ das variedades polares $P_{k}(|X \cap E|)$ é igual à multiplicidade em $x$ das variedades polares $P_{k}(X)$ para $0 \leqslant k \leqslant d-c-1$.

O próximo resultado é uma generalização do Teorema 5.19, para o caso de 3-variedades determinantais em $\mathbb{C}^{5}$.

Teorema 5.21. Seja $X \subset \mathbb{C}^{N}$ uma variedade determinantal de dimensão 3 com singularidade isolada, e seja $H$ um hiperplano em $\mathbb{C}^{N}$ dado pelo núcleo da projeção linear $p: \mathbb{C}^{N} \rightarrow \mathbb{C}$. Suponha que $X \cap H$ tem singularidade isolada na origem, então as seguintes condições são equivalentes:

a) pé geral a $X$ na origem

b) $\mu(X \cap H)$ é mínimo e $\mu\left(X \cap H \cap H^{\prime}\right)$ é mínimo para todo $H^{\prime}$ geral a $X$ e a $X \cap H$.

Demonstração: Suponha que $p: \mathbb{C}^{N} \rightarrow \mathbb{C}$ é geral a $X$ na origem, $H=p^{-1}(0)$ então $X \cap H$ é uma superfície determinantal com singularidade isolada na origem. Então existe um conjunto Zariski aberto $\Omega \subset \mathbb{P}^{N-1}$ tal que $\forall H^{\prime} \in \Omega$, com $H^{\prime}=\operatorname{ker} p^{\prime}, p^{\prime}: \mathbb{C}^{N} \rightarrow \mathbb{C}$ uma aplicação linear tem-se que:

1. $p$ é geral a $X$ na origem e $p$ é geral a $X \cap H^{\prime}$ na origem.

2. $\mu\left(X \cap H^{\prime}\right)$ é mínimo.

Usando a fórmula de tipo Lê- Greuel (3.2) da Proposição 3.12 para a superfície $X \cap H$ e $X \cap H^{\prime}$, temos:

$$
\mu(X \cap H)+\mu\left(X \cap H \cap H^{\prime}\right)=m_{2}(X \cap H)
$$




$$
\mu\left(X \cap H^{\prime}\right)+\mu\left(X \cap H \cap H^{\prime}\right)=m_{2}\left(X \cap H^{\prime}\right)
$$

Como $H$ e $H^{\prime}$ são gerais a $X$ em 0 , segue da Proposição 5.20 que $m_{2}(X \cap H)=m_{2}\left(X \cap H^{\prime}\right)=$ $m_{2}(X)$. Então segue das equações (5.2) e (5.3) que $\mu(X \cap H)=\mu\left(X \cap H^{\prime}\right)$, logo $\mu(X \cap H)$ é mínimo.

Sejam $H^{\prime}$ e $H^{\prime \prime}$ hiperplanos gerais a $X$ na origem, dados pelo kernel de $p^{\prime}$ e $p^{\prime \prime}$, tais que $\mu\left(X \cap H^{\prime} \cap H^{\prime \prime}\right)$ é mínimo. Então temos as seguintes fórmulas de tipo Lê- Greuel para $X \cap H$ e $X \cap H^{\prime}$.

$$
\begin{gathered}
\mu(X \cap H)+\mu\left(X \cap H \cap H^{\prime}\right)=m_{2}(X \cap H)=m_{2}(X) \\
\mu\left(X \cap H^{\prime}\right)+\mu\left(X \cap H^{\prime} \cap H^{\prime \prime}\right)=m_{2}\left(X \cap H^{\prime}\right)=m_{2}(X)
\end{gathered}
$$

Como $\mu(X \cap H)=\mu\left(X \cap H^{\prime}\right)$ então segue que $\mu\left(X \cap H \cap H^{\prime}\right)=\mu\left(X \cap H^{\prime} \cap H^{\prime \prime}\right)$.

Reciprocamente, seja $\pi=\left(p, p^{\prime}, p^{\prime \prime}\right)$ a aplicação $\mathbb{C}^{N} \rightarrow \mathbb{C}^{3}, \operatorname{com} H=\operatorname{ker} p, H^{\prime}=\operatorname{ker} p^{\prime}$ e $H^{\prime \prime}=\operatorname{ker} p^{\prime \prime}$, tal que ker $\pi \cap C_{X, 0}=\{0\}$ e $m_{0}(X)=\left.\operatorname{deg} \pi\right|_{X}$. Consideremos $\pi^{\prime}: \mathbb{C}^{3} \rightarrow \mathbb{C}^{2}$ tal que $\left.\pi^{\prime} \circ \pi\right|_{X \cap H}=\left(p^{\prime}, p^{\prime \prime}\right), \operatorname{ker}\left(\pi^{\prime} \circ \pi\right) \cap C_{X \cap H}=\{0\}$ e $m_{0}(X)=m_{0}(X \cap H)=\left.\operatorname{deg} \pi^{\prime} \circ \pi\right|_{X \cap H}$. Logo

$$
\begin{gathered}
\mu\left(X \cap H \cap H^{\prime}\right)+\operatorname{deg} \pi-1=m_{1}\left(X \cap H \cap H^{\prime}\right) \\
\mu\left(X \cap H^{\prime} \cap H^{\prime \prime}\right)+\operatorname{deg} \pi-1=m_{1}\left(X \cap H^{\prime} \cap H^{\prime \prime}\right) .
\end{gathered}
$$

Temos que $m_{1}(X)=m_{1}\left(X \cap H \cap H^{\prime}\right)$. Por outro lado, segue de [38, Lema 4.3] que

$$
\mu\left(X \cap H \cap H^{\prime}\right)+\operatorname{deg} \pi-1=\left(\Delta_{\pi^{\prime} \circ \pi} \cdot\left(\pi^{\prime} \circ \pi\right)\left(X \cap H \cap H^{\prime}\right)\right)_{0} .
$$

Logo $m_{1}\left(X \cap H \cap H^{\prime}\right)=\left(\Delta_{\pi^{\prime} \circ \pi} \cdot\left(\pi^{\prime} \circ \pi\right)\left(X \cap H \cap H^{\prime}\right)\right)_{0}$ então $\left(\pi^{\prime} \circ \pi\right)\left(X \cap H \cap H^{\prime}\right)$ não é limite de hiperplanos tangentes a $\Delta_{\pi^{\prime} \circ \pi}$. Logo pela Proposição 5.17, temos que $\pi(X \cap H)$ não é limite de hiperplanos tangentes a $\Delta_{\pi}$. Segue então pela Proposição 5.17 que $H$ não é limite de hiperplanos tangentes a $X$.

Exemplo 5.22. Seja $X$ a 3-variedade determinantal com singularidade isolada definida pela função

$$
\begin{aligned}
& F: \quad \mathbb{C}^{5} \quad \rightarrow \quad M_{2,3} \\
& (x, y, z, w, v) \mapsto\left(\begin{array}{ccc}
x & y & z \\
w & v & x^{2}+y^{2}
\end{array}\right)
\end{aligned}
$$

Sejam p e $p^{\prime}$ as projeções lineares definidas por

$$
p(x, y, z, w, v)=w-z
$$


$e$

$$
p^{\prime}(x, y, z, w, v)=x-v
$$

que definem os hiperplanos $H$ e $H^{\prime}$ respectivamente.

Então a superfície $X \cap H$ é determinada pela seguinte matriz

$$
\left(\begin{array}{ccc}
x & y & z \\
z & v & x^{2}+y^{2}
\end{array}\right)
$$

O número de Milnor de $X \cap H$ é $\mu(X \cap H)=4$, ver [34].

A superfície $X \cap H^{\prime}$ é determinada pela seguinte matriz

$$
\left(\begin{array}{ccc}
x & y & z \\
w & x & x^{2}+y^{2}
\end{array}\right) \sim_{\mathcal{G}(n, m)}\left(\begin{array}{ccc}
x & y & z \\
w & x & y^{2}
\end{array}\right)
$$

Então segue de [35] que o número de Milnor desta superfície é $\mu\left(X \cap H^{\prime}\right)=2$.

Então temos que $p^{\prime}$ é geral a X na origem.

\subsection{Seções de Variedades Determinantais Essencialmente Iso- ladas}

Quando $X$ é uma EIDS, de dimensão $d>2$ também é possível associar a $X$ uma superfície determinantal $Y$ cujo número de Milnor é um invariante de $X$.

Proposição 5.23. [26] Sejam $H$ e $H^{\prime}$ hiperplanos em $\mathbb{C}^{N}$ que não são limites de hiperplanos tangentes a $(X, 0)$. Então $H$ e $H^{\prime}$ contêm planos $Q$ e $Q^{\prime}$ respectivamente tais que as curvas reduzidas $|X \cap Q| e\left|X \cap Q^{\prime}\right|$ têm o mesmo número de Milnor.

O seguinte resultado é uma generalização da Proposição 5.23 e resulta como consequiência da Proposição 5.20 e da fórmula de tipo Lê- Greuel, Teorema 3.3 .

Teorema 5.24. Seja $X \subset \mathbb{C}^{N}$ uma EIDS de tipo $(m, n, t)$ de dimensão d. Sejam $H$ e $H^{\prime}$ hiperplanos de $\mathbb{C}^{N}$ fortemente gerais para $(X, 0)$ na origem. Então $H$ e $H^{\prime}$ contêm respetivamente $P$ e $P^{\prime}$ tais que codim $P=\operatorname{codim} P^{\prime}=d-2$, para os quais as superfícies determinantais $X \cap P$ e $X \cap P^{\prime}$ satisfazem as seguintes condições

a) têm singularidade isolada

b) admitem suavizações

c) $\mu(X \cap P)=\mu\left(X \cap P^{\prime}\right)$.

Demonstração: 
a) Podemos considerar $P=H \cap \cdots \cap H_{d-3} \subset H$ e $P^{\prime}=H^{\prime} \cap \cdots \cap H_{d-3}^{\prime} \subset H^{\prime}$ tais que para tudo $i, H_{i}$ e $H_{i}^{\prime}$ são fortemente gerais para $X \cap H \cap H_{1} \cap \cdots \cap H_{i-1}$ e $X \cap H^{\prime} \cap H_{1}^{\prime} \cap \cdots \cap H_{i-1}^{\prime}$ respectivamente. Pela Proposição 5.12, segue que $X \cap P \subset \mathbb{C}^{N-d+2}$ e $X \cap P^{\prime} \subset \mathbb{C}^{N-d+2}$ são EIDS e como a codim $X<(m-t+2)(n-t+2)-2$ então $X \cap P$ e $X \cap P^{\prime}$ são superfícies determinantais com singularidade isolada.

b) Seja $\mathcal{X}$ um smoothing essencial de $X$, tal que $H_{i} \times \mathbb{C}$ e $H_{i}^{\prime} \times \mathbb{C}$ são fortemente gerais para $\mathcal{X} \cap(H \times \mathbb{C}) \cap\left(H_{1} \times \mathbb{C}\right) \cap \cdots \cap\left(H_{i-1} \times \mathbb{C}\right)$ e $\mathcal{X} \cap\left(H^{\prime} \times \mathbb{C}\right) \cap\left(H_{1}^{\prime} \times \mathbb{C}\right) \cap \ldots\left(\cap H_{i-1}^{\prime} \times \mathbb{C}\right)$ respetivamente. De fato, $\mathcal{X} \cap \widetilde{P}$ e $\mathcal{X} \cap \widetilde{P}^{\prime}$, com $\widetilde{P}=P \times \mathbb{C}$ e $\widetilde{P}^{\prime}=P^{\prime} \times \mathbb{C}$ são as suavizações de $X \cap P$ e $X \cap P^{\prime}$, pelo Teorema 2.8 .

c) Os hiperplanos $H$ e $H^{\prime}$ contêm planos $Q=P \cap H^{\prime}$ e $Q^{\prime}=P^{\prime} \cap H$ dados na Proposição 5.23, então $X \cap Q$ e $X \cap Q^{\prime}$ são curvas determinantais e pela Proposição 5.23, temos que:

$$
\mu(X \cap Q)=\mu\left(X \cap Q^{\prime}\right)
$$

Usando a fórmula de tipo Lê- Greuel, Teorema 3.3, temos

$$
\begin{aligned}
\mu(X \cap P)+\mu(X \cap Q) & =m_{2}\left(X \cap P, p^{\prime}\right) \\
\mu\left(X \cap P^{\prime}\right)+\mu\left(X \cap Q^{\prime}\right) & =m_{2}\left(X \cap P^{\prime}, p\right) .
\end{aligned}
$$

Podemos aplicar a Proposição 5.20 na variedade $\mathcal{X} \subset \mathbb{C}^{N} \times \mathbb{C}$, e nos hiperplanos produto $H_{i} \times \mathbb{C}$. Logo, temos que $m_{2}\left(X \cap P, p^{\prime}\right)=m_{2}\left(X \cap P^{\prime}, p\right)$ pois $m_{2}\left(X \cap P, p^{\prime}\right)=$ $m_{0}\left(P_{2}(X \cap P), p^{\prime}\right)=m_{0}\left(P_{2}(\mathcal{X})\right)$, e da mesma forma $m_{2}\left(X \cap P^{\prime}, p\right)=m_{0}\left(P_{2}(\mathcal{X})\right)$. Logo $m_{2}\left(X \cap P, p^{\prime}\right)=m_{2}\left(X \cap P^{\prime}, p\right)=m_{0}\left(P_{2}(\mathcal{X})\right)$ e usando 5.8) temos o resultado.

Exemplo 5.25. Seja $F: \mathbb{C}^{N} \rightarrow M_{2,3}, N \geqslant 7$ definida por

$$
F(x, y)=\left(\begin{array}{ccc}
x_{1} & x_{2} & x_{3} \\
x_{4} & x_{5} & x_{6}+f(y)
\end{array}\right), f(0)=0
$$

onde $x=\left(x_{1}, x_{2}, \ldots, x_{6}\right)$ e $y=\left(y_{1}, \ldots, y_{N-6}\right)$. Então $X=F^{-1}\left(M_{m, n}^{t}\right)$ é uma EIDS em $\mathbb{C}^{N}$ de codimensão 2. Considere $P=\left\{(x, y) \mid x_{1}=x_{5}, x_{2}=x_{6}, y=0\right\}$, obtemos a superfície determinantal em $\mathbb{C}^{4}$ definida por

$$
\left(\begin{array}{lll}
x_{1} & x_{2} & x_{3} \\
x_{4} & x_{1} & x_{2}
\end{array}\right)
$$

Segue de [34] que $\mu(X \cap P)=1$. 


\section{Topologia de EIDS}

Neste capítulo estudamos o conceito de índice de Poincaré- Hopf de 1-formas sobre EIDS e estudamos a noção de obstrução de Euler local de variedades analíticas. O principal objetivo é calcular a obstrução de Euler de variedades determinantais essencialmente isoladas definidas pela função $F: \mathbb{C}^{N} \rightarrow M_{m, n}$ e que admitem 3 estratos. Daremos algumas condições sobre $N$ para garantir a existência de tais variedades e forneceremos uma fórmula explícita da obstrução de Euler em termos da característica de Euler evanescente, definida em [12]. Além disso estudaremos o caso particular das EIDS, definidas por uma função $F: \mathbb{C}^{N} \rightarrow M_{2,3}$ de coposto 1 . Neste caso, verificaremos que a obstrução de Euler é constante.

\subsection{Indices de Poincaré Hopf de 1-formas sobre EIDS}

Nesta seção, com base no artigo [23], definimos índices de 1-formas sobre variedades determinantais. Estes índices são índices de Poincaré Hopf, e são definidos usando as três resoluções estudadas no Capítulo 4.

Seja $(X, 0)=F^{-1}\left(M_{m, n}^{t}\right) \subset\left(\mathbb{C}^{N}, 0\right)$ de tipo $(m, n, t)$ e $w$ uma 1 -forma sobre $\left(\mathbb{C}^{N}, 0\right)$ tal que $\left.w\right|_{X}$ tem um ponto singular isolado na origem.

Uma suavização essencial $\widetilde{X}=\widetilde{F}^{-1}\left(M_{m, n}^{t}\right) \subset U$ de EIDS $(X, 0)$ em geral não é suave. Para definir um análogo ao índice de Poincaré-Hopf, temos que construir um substituto para o fibrado tangente a $\widetilde{X}$.

Uma possibilidade é usar uma resolução $\widetilde{X}$ de $X$, usando uma das resoluções $Y_{k}, k=1,2,3$ de $M_{m, n}^{t}$, apresentadas no Capítulo 4. Nestes casos, $\bar{X}_{k}$ é o pull-back de $\left(\pi_{k}, \widetilde{F}\right)$, no diagrama

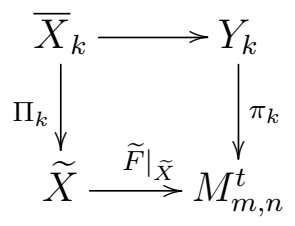


Vimos no Capítulo 4 que $\bar{X}_{k}=\widetilde{X} \times_{M_{m, n}^{t}} Y_{k}$ é suave. Como $w$ é uma forma sobre $\mathbb{C}^{N}$ então podemos definir uma 1-forma sobre $\bar{X}_{k}$ como: $\left(j \circ \Pi_{k}\right)^{*}(w)=w_{k}$ (onde $j$ é uma aplicação inclusão $\widetilde{X} \hookrightarrow U \subset \mathbb{C}^{N}$ ).

Temos que $w_{k}$ é uma 1-forma sobre a variedade analítica complexa não singular $\bar{X}_{k}$ sem zeros fora da preimagem de uma vizinhança na origem. Em geral $w_{k}$ pode ter zeros não isolados. Podemos ver $w_{k}$ como uma seção do fibrado cotangente de $\bar{X}_{k}$.

Definição 6.1. [23] $O$ índice de Poincaré-Hopf (PH-índice) $i n d_{P H}^{k}(w)=i n d_{P H}^{k}(w, X, 0)$, $k=1,2,3$, da 1-forma $w$ sobre a $\operatorname{EIDS}(X, 0) \subset\left(\mathbb{C}^{N}, 0\right)$ é a soma dos índices dos zeros de uma perturbação genérica $\widetilde{w}_{k}$ da 1-forma $w_{k}$ sobre a variedade $\bar{X}_{k}$. Em outras palavras, o PH-índice $i n d_{P H}^{k}(w)$ é a obstrução à extensão da 1 - forma não nula $w_{k}$ da preimagem (sob $j \circ \Pi_{k}$ ) da vizinhança da esfera $S_{\epsilon}=\partial B_{\epsilon}$ à variedade $\bar{X}_{k}$.

$$
\operatorname{ind}_{P H}^{k}(w)=\operatorname{Obs}\left(w_{k},\left(j \circ \Pi_{k}\right)^{-1}\left(B_{\epsilon}\right),\left(j \circ \Pi_{k}\right)^{-1}\left(S_{\epsilon}\right)\right)
$$

Este índice independe da escolha da deformação.

Outra possibilidade é levar em conta que o espaço $\bar{X}_{3}$ da terceira resolução é a transformação de Nash de $\tilde{X}$. Vamos usar o fibrado de Nash $\widehat{\mathbb{T}}$ de $\tilde{X}$ no lugar do fibrado tangente de $\bar{X}_{k}$. A 1-forma $w$ define uma seção não nula $\widehat{w}$ do fibrado dual de $\widehat{T}^{*}$ sobre a preimagem da intersecção de $\tilde{X} \cap S_{\epsilon}$.

Definição 6.2. [23] O índice de Poincaré-Hopf-Nash (PHN-índice) da 1-forma w sobre $(X, 0)$ $\operatorname{ind}_{P H N} w=\operatorname{ind}_{P H N}(w, X, 0)$ é a obstrução a extensão da seção não nula $\widehat{w}$ do fibrado dual $\widehat{\mathbb{T}}^{*}$ da preimagem da esfera $S_{\epsilon}=\partial B_{\epsilon} \grave{a}$ preimagem do seu interior, ie, à variedade $\bar{X}_{3}$ sobre a classe fundamental do par $\left[\left(\Pi_{3}^{-1}\left(X \cap B_{\epsilon}\right), \Pi_{3}^{-1}\left(X \cap S_{\epsilon}\right)\right)\right]$.

$$
\operatorname{ind}_{P H N} w=\operatorname{Obs}\left(\widehat{w}, \Pi_{3}^{-1}\left(X \cap B_{\epsilon}\right), \Pi_{3}^{-1}\left(X \cap S_{\epsilon}\right)\right)\left(\left[\left(\Pi_{3}^{-1}\left(X \cap B_{\epsilon}\right), \Pi_{3}^{-1}\left(X \cap S_{\epsilon}\right)\right)\right]\right)
$$

Proposição 6.3. [23] $O$ indice de PHN, ind ${ }_{P H N}(w)=\operatorname{ind}_{P H N}(w ; X, 0)$ da 1-forma $w$ sobre uma EIDS $X$, é igual ao número de pontos singulares não degenerados de uma deformação genérica $\widetilde{w}$ da 1 - forma $w$ sobre a parte não singular $\widetilde{X}_{\text {reg }}=F^{-1}\left(M_{m, n}^{t} \backslash M_{m, n}^{t-1}\right)$ de uma suavização essencial $\widetilde{X}$ da variedade $(X, 0)$.

Quando $X$ é uma variedade determinantal com singularidade isolada que admite uma suavização e $w$ é uma 1 -forma definida em $X$, o número de pontos singulares não degenerados de uma perturbação $\widetilde{w}$ de $w$ sobre a suavização $\widetilde{X}$ de $X$ é o índice GSV de $w$ sobre $X$ em $x=0$, ver [5] para mais detalhes.

Então temos o seguinte corolário da proposição anterior. 
Corolário 6.4. Se X é IDS que admite suavização e wé uma 1-forma em X com singularidade isolada em $x=0$. Então

$$
\operatorname{ind}_{P H N}(w, X, 0)=\operatorname{ind}_{G S V}(w, X, 0)
$$

Queremos mostrar que para o caso de uma variedade com suavização e com singularidade isolada todos os índices de Poincaré Hopf ( e o índice de Poincaré- Hopf- Nash) coincidem. Temos o seguinte resultado enunciado por Èbeling e Guseřn-Zade [23, pag. 120].

Teorema 6.5. Seja X uma variedade determinantal com singularidade isolada, que admite uma suavização $\widetilde{X}$. Então

$$
\operatorname{Ind}_{P H}^{3}(w, X, 0)=\operatorname{Ind}_{P H N}(w, X, 0)
$$

Demonstração: Seja $w$ uma 1-forma definida sobre $\mathbb{C}^{N}$ tal que $\left.w\right|_{X}$ tem singularidades isolada na origem, como $X$ é uma variedade singular com suavização, temos que a suavização é uma suavização essencial $\widetilde{X}$ ( é suave ), assim $\bar{X}_{3} \cong \widetilde{X}$, então temos $\widehat{\mathbb{T}}$ é o fibrado tangente de $\widetilde{X}$.

Seja $\chi(X, 0)=\chi\left(\widetilde{X} \cap B_{\epsilon}\right)$, onde $\widetilde{X}$ é uma suavização essencial de $(X, 0)$. Denotemos por $\widetilde{X}_{i} \subset \widetilde{X}$ a suavização essencial da $\operatorname{EIDS} X_{i}=F^{-1}\left(M_{m, n}^{i}\right)$ de tipo $(m, n, i)$.

Vamos denotar por $G_{i}^{k}$ a imagem inversa de um ponto de $V_{i}=M_{m, n}^{i} \backslash M_{m, n}^{i-1}$ das resoluções $\pi_{k}$ da variedade $M_{m, n}^{t}$.

$$
\begin{aligned}
G_{i}^{1} & =G r(n-t+1, n-i+1) \\
G_{i}^{2} & =G r(m-t+1, m-i+1) \\
G_{i}^{3} & =\operatorname{Gr}(n-t+1, n-i+1) \times G r(m-t+1, m-i+1)
\end{aligned}
$$

Definiremos o índice radial de 1- formas complexas com base em [5, 14].

Seja $(X, 0) \subset\left(\mathbb{R}^{N}, 0\right)$ um germe de variedade analítica e seja $w$ uma 1 - forma sobre uma vizinhaça na origem em $\mathbb{R}^{N}$.

Definição 6.6. [14] A 1- forma $w$ é radial sobre $(X, 0)$ se para qualquer curva analítica $\varphi$ : $(\mathbb{R}, 0) \rightarrow(X, 0)$, o valor da 1-forma sobre o espaço tangente $\dot{\varphi}$ é positivo para $t>0$.

Seja $X=\cup_{i=0}^{q} V_{i}$ uma estratificação de Whitney do germe $(X, 0), V_{0}=\{0\}$. Seja $w$ uma 1-forma sobre uma vizinhança na origem em $\mathbb{R}^{N}$, com uma singularidade isolada sobre $(X, 0)$ na origem. Seja $\epsilon>0$ suficientemente pequeno tal que na bola fechada $B_{\epsilon}$ de raio $\epsilon$ e centrada na origem em $\mathbb{R}^{N}$ a 1-forma $w$ não tem pontos singulares sobre $X \backslash\{0\}$. Então existe uma 1forma $\widetilde{w}$ sobre $\mathbb{R}^{N}$ tal que 
1. A 1- forma $\widetilde{w}$ coincide com a 1-forma sobre uma vizinhança da esfera $S_{\epsilon}=\partial B_{\epsilon}$.

2. A 1- forma $\widetilde{w}$ é radial sobre $(X, 0)$ na origem.

3. Em cada vizinhaça de um ponto singular $x_{0} \in\left(X \cap B_{\epsilon}\right) \backslash\{0\}, x_{0} \in V_{i}, \operatorname{dim} V_{i}=k$, a 1- forma $\widetilde{w}$ pode ser vista como segue. Existe um difeomorfismo analítico local $h$ : $\left(\mathbb{R}^{N}, \mathbb{R}^{k}, 0\right) \rightarrow\left(\mathbb{R}^{N}, V_{i}, x_{0}\right)$ tal que $h^{*} \widetilde{w}=\pi_{1}^{*} \widetilde{w}_{1}+\pi_{2}^{*} \widetilde{w}_{2}$, onde $\pi_{1}: \mathbb{R}^{N} \rightarrow \mathbb{R}^{k}$ e $\pi_{2}:$ $\mathbb{R}^{N} \rightarrow \mathbb{R}^{N-k}$ são projeções, $\widetilde{w}_{1}$ é o germe de 1 -forma sobre $\left(\mathbb{R}^{k}, 0\right)$ com um ponto singular isolado na origem e $\widetilde{w}_{2}$ é uma forma radial sobre $\left(\mathbb{R}^{N-k}, 0\right)$.

Definição 6.7. $O$ índice radial da 1-forma $w$ sobre a variedade $X$ na origem é

$$
i n d_{\text {rad }}(w ; X, 0)=1+\left.\sum_{i=1}^{q} \sum_{Q \in \Sigma \widetilde{w} \cap V_{i}} i n d_{Q} \widetilde{w}\right|_{V_{i}},
$$

onde a segunda soma é considerada sobre todos os pontos singulares da 1-forma $\widetilde{w}$ sobre $V_{i} \cap B_{\epsilon}$.

Agora seja $(X, 0) \subset\left(\mathbb{C}^{N}, 0\right)$ um germe de variedade analítica complexa de dimensão $d$ e seja $w$ uma 1- forma complexa sobre uma vizinhança da origem em $\mathbb{C}^{N}$. Existe uma correspondência entre as 1-formas complexas sobre $\mathbb{C}^{N}$ e 1 -formas reais sobre $\mathbb{C}^{N}$ (considerando $\mathbb{C}^{N}$ como uma variedades real de dimensão $2 N$ ). Ou seja, a uma 1- forma complexa $w$ associamos uma 1- forma real $\eta=R e w$; a 1 forma $w$ pode ser obtida pela fórmula $w(v)=\eta(v)-i \eta(i v)$ para $v \in T_{x} \mathbb{C}^{N}$. Isto quer dizer que o índice radial à 1 - forma real $R e W$ é um invariante da 1 - forma complexa sobre si mesma. Porém, sobre uma variedade suave $M d$-dimensional, $i_{n d} d_{\text {rad }}(\operatorname{Rew} ; M, x)$ não coincide com o índice usual de um ponto singular $x$ da 1 - forma $w$. Eles diferem pelo coeficiente $(-1)^{d}$.

Definição 6.8. [5] 14] O indice radial da 1-forma complexa w sobre $X$ na origem, ind $_{\text {rad }}(w ; X, 0)$, é $\operatorname{ind}_{\text {rad }}(w ; X, 0)=(-1)^{d}$ ind $_{\text {rad }}(\operatorname{Rew} ; X, 0)$.

Proposição 6.9. [23] Temos a seguinte igualdade:

$$
\begin{aligned}
i n d_{P H}^{k}(w ; X, 0)=(-1)^{\operatorname{dim} X} & \sum_{i=1}^{t}\left((-1)^{\operatorname{dim} X_{i}} i n d_{r a d}\left(w ; X_{i}, 0\right)-(-1)^{\operatorname{dim} X_{i-1}} i n d_{\text {rad }}\left(w ; X_{i-1}, 0\right)\right. \\
& \left.+\left(\chi\left(X_{i}, 0\right)-\chi\left(X_{i-1}, 0\right)\right)\right) \chi\left(G_{i}^{k}\right),
\end{aligned}
$$

onde $X_{0}=\{0\}$ e ind $\operatorname{rad}_{\text {rad }}\left(w, X_{0}, 0\right)=1$ e $\chi\left(X_{0}, 0\right)=0$.

Calculamos a diferença entre o índices de Poincaré Hopf e o índice de Poincré Hopf Nash. Queremos usar esta diferença para exibir um exemplo em que os fibrados correspondentes, fibrado de Nash e fibrado tangente da transformação de Nash são isomorfos se a transformada de Nash é suave. 
Usando as fórmulas dadas na proposição 6. no artigo [23], temos a seguinte diferença:

$$
i n d_{P H}^{k}(w)-i n d_{P H N}(w)=\left[(-1)^{\operatorname{dim} X} \bar{\chi}\left(G_{t-1}^{k}\right)+(-1)^{m+n+2}(m-t+1)\right] \chi\left(X_{t-1}, 0\right),
$$

onde $\bar{\chi}(X)=\chi(X)-1$.

\subsection{Obstrução de Euler Local}

Sejam $(X, 0) \subset\left(\mathbb{C}^{N}, 0\right)$ germe de variedade analítica complexa, $X$ um representante suficientemente pequeno do germe $(X, 0)$ e $\left\{V_{i}\right\}$ uma estratificação de Whitney de $X$. Suponhamos que $\{0\}$ seja um estrato. Seja $\cup T V_{i}$ a união dos fibrados tangentes a todos os estratos. Esta união pode ser vista como um subconjunto do fibrado tangente $\left.T \mathbb{C}^{N}\right|_{X}$. A referência para esta seção são os artigos [3, 2, 4].

Definição 6.10. Seja $v$ uma seção de $T \mathbb{C}^{N}$ sobre um subconjunto $A \subset \mathbb{C}^{N}$. Dizemos que o campo $v$ é estratificado se para $z \in V_{i} \cap A$, temos que $v(z) \in T_{z} V_{i}$, onde $V_{i}$ é o estrato que contém z.

Definição 6.11. Dizemos que o campo de vetores $v$ é radial em a se vé um campo estratificado e se existe $\epsilon_{0}>0$ tal que o campo v está saindo de $B_{\epsilon}(a)$, para $0<\epsilon \leqslant \epsilon_{0}$.

Lema 6.12. [4] Um campo de vetores estratificado v não nulo definido sobre um subconjunto $A \subset X$ admite um levantamento canônico v como seção não nula do fibrado de Nash, $\tilde{T}$, sobre $\nu^{-1}(A)$.

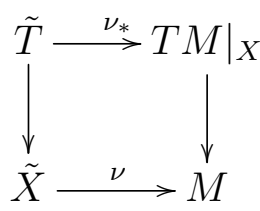

\section{Demonstração:}

Dado $\tilde{x} \in \tilde{X}$, denotemos por $x=\nu(\tilde{x})$

1. Se $x \in X_{\text {reg }}$, então $v(x) \in T_{x}\left(X_{\text {reg }}\right)=\tilde{x} \operatorname{com} \tilde{x}=\nu^{-1}(x)$. Então $\tilde{v}(\tilde{x})=\left(x, T_{x}\left(X_{r e g}\right), v(x)\right)$.

2. Se $x \in V_{i}$, então $v(x) \in T_{x}\left(V_{i}\right)$. Temos que cada $x \in \nu^{-1}(x)$ está no fecho da imagem do $s$, ver Definição 1.9 . Então existe uma sequência $\left(\tilde{x}_{n}\right)$ de pontos de $\tilde{X}_{\text {reg }}$ tal que $\tilde{x}=\lim \tilde{x}_{n}, \nu\left(\tilde{x}_{n}\right)=x_{n} \in X_{\text {reg }}$ e $\tilde{x}_{n}=T_{x_{n}}\left(X_{r e g}\right)$. Então temos que $\lim x_{n}=x$ e $\lim T_{x_{n}}\left(X_{r e g}\right)=\tilde{x}$. Como $\left\{V_{i}\right\}$ é uma estratificação de Whitney, temos pela condição $\left.a\right)$ da Definição 1.7 que $T_{x}\left(V_{i}\right) \subset \tilde{x}$. E então $\tilde{v}(\tilde{x})=(x, \tilde{x}, v(x))$ está bem definido. 
A definição de obstrução de Euler foi dada por R. MacPherson [29]. Aqui damos uma definição equivalente dada por J.-P. Brasselet e M.-H. Schwartz.

Definição 6.13. [4] Seja v um campo de vetores radial sobre $X \cap \partial B_{\epsilon}$ e v o levantamento de $v$ sobre o conjunto $\nu^{-1}\left(X \cap \partial B_{\epsilon}\right)$. O campo ṽ define um cociclo de obstrução Obs $(\tilde{v})$, que mede a obstrução para estender $\tilde{v}$ como seção não-nula de $\tilde{T}$ sobre $\nu^{-1}\left(X \cap B_{\epsilon}\right)$ :

$$
\operatorname{Obs}(\tilde{v}) \in Z^{2 N}\left(\nu^{-1}\left(X \cap B_{\epsilon}\right), \nu^{-1}\left(V \cap \partial B_{\epsilon}\right)\right)
$$

A obstrução de Euler local, denotada por $E u_{X}(0)$ é a avaliação do cociclo Obs $(\tilde{v})$ sobre a classe fundamental $\left[\nu^{-1}\left(X \cap B_{\epsilon}\right), \nu^{-1}\left(V \cap \partial B_{\epsilon}\right)\right]$, quer dizer:

$$
E u_{0}(X)=\left\langle\operatorname{Obs}(\tilde{v}),\left[\nu^{-1}\left(X \cap B_{\epsilon}\right), \nu^{-1}\left(V \cap \partial B_{\epsilon}\right)\right]\right\rangle
$$

Observação 6.14. As seguintes propriedades da obstrução de Euler se verificam:

- Eu $u_{x}(X)$ é constante sobre os estratos de uma estratificação de Whitney de X.

- $E u_{x}(X)=1$, se $x \in X_{\text {reg. }}$.

- Assuma que $X$ é localmente mergulhado em $\mathbb{C}^{N}$ e $W$ uma variedade suave em $\mathbb{C}^{N}$ que intercepta a estratificação de Whitney de $X$ transversalmente. Então E $u_{x}(X \cap W)=$ $E u_{x}(X)$ para todo ponto $x \in X \cap W$.

- $E u_{(x, y)}(X \times Y)=E u_{x}(X) \times E u_{y}(Y)$ para $x \in X$ e $y \in Y$.

O teorema seguinte é a fórmula de tipo Lefschetz para a obstrução de Euler, dada em [3, Teorema 3.1]. Este resultado é muito importante para o cálculo da obstrução de Euler.

Teorema 6.15. [3] Seja $(X, 0)$ um germe de espaço analítico complexo equidimensional em $\mathbb{C}^{N}$. Seja $V_{i}, i=1, \ldots, l$ estratos de uma estratificação de Whitney de um representante pequeno $X$ de $(X, 0)$. Então existe um subconjunto aberto Zariski $\Omega$ no espaço de formas lineares complexas sobre $\mathbb{C}^{N}$, tal que para cada $l \in \Omega$, existe $\epsilon_{0}$, tal que para cada $\epsilon, \epsilon_{0}>\epsilon>0$ e $t_{0} \neq 0$ suficientemente pequeno tal que a seguinte fórmula para a obstrução de Euler de $(X, 0)$ se verifica.

$$
E u_{0}(X)=\sum_{1}^{l} \chi\left(V_{i} \cap B_{\epsilon} \cap l^{-1}\left(t_{0}\right)\right) E u_{V_{i}}(X),
$$

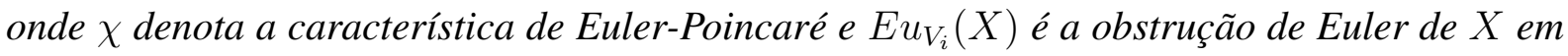
qualquer ponto de $V_{i}, i=1, \ldots, l$.

Para o cálculo da obstrução de Euler de variedades determinantais, a seguinte fórmula dada em [23] é bastante útil. Para variedades determinantais $X=F^{-1}\left(M_{m, n}^{t}\right)$ fora da ori- 
gem, uma seção normal ao estrato $V_{i}=X_{i} \backslash X_{i-1}$ (onde $X_{i}=F^{-1}\left(M_{m, n}^{i}\right)$ ) é isomorfo a $\left(M_{m-i+1, n-i+1}^{t-i+1}, 0\right)$.

Teorema 6.16. [23] Seja $l: M_{m, n} \rightarrow \mathbb{C}$ uma forma linear genérica, e seja $L_{m, n}^{t}=M_{m, n}^{t} \cap$ $l^{-1}(1)$. Então, para $t \leqslant m \leqslant n$, se tem:

$$
\bar{\chi}\left(L_{m, n}^{t}\right)=(-1)^{t}\left(\begin{array}{c}
m-1 \\
t-1
\end{array}\right),
$$

onde $\bar{\chi}\left(L_{m, n}^{t}\right)=\chi\left(L_{m, n}^{t}\right)-1$.

\subsection{Número de Milnor Singular de Seções de Interseções Com- pletas}

Definição 6.17. [12] Sejam $F:\left(\mathbb{C}^{N}, 0\right) \rightarrow\left(\mathbb{C}^{p}, 0\right)$ uma função analítica e $(V, 0) \subset\left(\mathbb{C}^{p}, 0\right)$ um germe de conjunto analítico. Dizemos que $F$ é geometricamente transversal a $(V, 0)$ fora da origem se existe, $U=B_{\epsilon}(0) \backslash\{0\} \subset \mathbb{C}^{N}$ tal que $F$ é transversal a todos os estratos de $V, \forall$ $x \in U$.

Definição 6.18. [12] Seja $(V, 0) \subset\left(\mathbb{C}^{p}, 0\right)$ um germe de uma conjunto analítico. Sejam $\eta_{1}, \ldots, \eta_{k}$ o conjunto de geradores para o $\operatorname{Derlog}(V)$. Uma função $F: \mathbb{C}^{N} \rightarrow \mathbb{C}^{p}$ é algebricamente transversal a $(V, 0)$ em $x_{0} \in \mathbb{C}^{N}$ se

$$
d_{x_{0}} F\left(T_{x_{0}} \mathbb{C}^{N}\right)+\langle\operatorname{Derlog}(V)\rangle_{F\left(x_{0}\right)}=T_{F\left(x_{0}\right)} \mathbb{C}^{P},
$$

onde para $y \in \mathbb{C}^{p},\langle\operatorname{Derlog}(V)\rangle_{y}=\mathbb{C}\left\{\eta_{1}(y), \ldots, \eta_{k}(y)\right\} \subset T_{y} \mathbb{C}^{p}$. Se existe uma vizinhança $U=B_{\epsilon}(0) \backslash\{0\} \subset \mathbb{C}^{N}$ tal que $F$ é algebricamente transversal a $(V, 0)$ em $x \in U$, então dizemos que $F$ é algebricamente transversal a $(V, 0)$ fora da origem.

Seja $F:\left(\mathbb{C}^{N}, 0\right) \rightarrow\left(\mathbb{C}^{p}, 0\right)$ um germe holomorfo e seja $(V, 0) \subset\left(\mathbb{C}^{p}, 0\right)$ uma interseção completa de dimensão $k$. Seja $F^{\prime}:\left(\mathbb{C}^{N} \times \mathbb{C}, 0\right) \rightarrow\left(\mathbb{C}^{p}, 0\right)$ um desdobramento versal de $F$ definido sobre uma vizinhança $U \times T \subset \mathbb{C}^{N} \times \mathbb{C}$. Denotemos por $F_{t}(x)=F^{\prime}(x, t)$.

Definição 6.19. [12] Sejam F e V como acima, assuma que F é geometricamente transversal a $V$ fora da origem. Então chamamos $F^{\prime}$ uma estabilização geométrica com relação a $V$ se para todo $t \in T \backslash\{0\}, F_{t}$ é geometricamente transversal a $V$ sobre $U$.

Definição 6.20. Sejam $F$ e $V$ como acima, assuma que $F$ é algebricamente transversal a $V$ fora da origem. Então $F^{\prime}$ é uma estabilização algébrica de $F$ com relação a $V$ se para todo $t \in T \backslash\{0\}, F_{t}$ é algebricamente transversal a $V$ sobre $U$. 
Proposição 6.21. [12] Sejam $F:\left(\mathbb{C}^{N}, 0\right) \rightarrow\left(\mathbb{C}^{p}, 0\right)$ um germe analítico e $(V, 0) \subset\left(\mathbb{C}^{p}, 0\right)$ um conjunto analítico

1. F tem $\mathcal{K}_{V, e^{-}}$codimensão finita se e somente se $F$ é algebricamente transversal a $V$ fora da origem.

2. Seja $H: \mathbb{C}^{p} \rightarrow \mathbb{C}$ um germe analítico tal que $V=H^{-1}(0)$. Então $F$ tem $\mathcal{K}_{H, e^{-}}$codimensão finita se e somente se $F$ é algebricamente transversal a $V=H^{-1}(0)$ fora da origem.

Teorema 6.22. $[10,11,12]$ Seja $F:\left(\mathbb{C}^{N}, 0\right) \rightarrow\left(\mathbb{C}^{p}, 0\right)$ um germe holomorfo e seja $(V, 0) \subset$ $\left(\mathbb{C}^{p}, 0\right)$ uma interseção completa de dimensão $k$. Suponha que $F$ é geometricamente transversal a $V$ fora da origem. Seja $F^{\prime}:\left(\mathbb{C}^{N} \times \mathbb{C}, 0\right) \rightarrow\left(\mathbb{C}^{p}, 0\right)$ uma estabilização geométrica de $F$ com relação a $V$ e seja $F_{t}=F^{\prime}(\cdot, t)$. Então existem $\epsilon>0$ e $0<\delta \ll \epsilon$ tais que se $0<|t|<\delta$, a fibra $F_{t}^{-1}(V) \cap B_{\epsilon}(0)$ tem o tipo de homotopia de um bouquet de esferas de dimensão real $N-p+k$, e o número de esferas é independente da escolha da estabilização de te $\epsilon$.

Definição 6.23. [12] $O$ conjunto $F_{t}^{-1}(V) \cap B_{\epsilon}$ é chamado fibra de Milnor singular de $F$ com relação a $V$ (ou a $X=F^{-1}(V)$ ). O número de esferas é chamado número de Milnor singular de $X$ e é denotado por $\mu_{V}(F)$ ou $\mu(X)$.

Definição 6.24. Seja $(V, 0) \subset\left(\mathbb{C}^{p}, 0\right)$ uma hipersuperfície. Dizemos que $V$ é um divisor livre se Derlog $(V)$ é um $\mathcal{O}_{\mathbb{C}^{p}, 0}$-módulo livre de posto $p$.

Um divisor livre $V$ é chamado holonômico se para cada $z \in V$ os geradores do Derlog $(V)$ avaliados em z geram o espaço tangente do estrato correspondente da estratificação de Whitney de $V$ que contém z. Se $H$ é a equação que define $V$ e Derlog $(H)$ satisfaz a condição acima, dizemos que $V$ é $H$ - holonômico.

Teorema 6.25. [12] Teorema 3.1] Se $V \subset \mathbb{C}^{p}$ é um divisor livre, H holonômico e $F:\left(\mathbb{C}^{N}, 0\right) \rightarrow$ $\mathbb{C}^{p}, 0$ é transversal a $V$ fora da origem, então

$$
\mu(X)=\mathcal{K}_{H, e}-\operatorname{codim}(F)
$$

onde $\mathcal{K}_{H, e}$ é a $\mathcal{K}_{H, e}$ codimensão de $F$.

Definição 6.26. [12] Uma hipersuperfície singular $(W, 0) \subset\left(\mathbb{C}^{p}, 0\right)$ admite um completamento livre se existe um divisor livre $(V, 0) \subset\left(\mathbb{C}^{p}, 0\right)$ talque $V \cup W$ é outra vez um divisor livre. 


\subsection{A característica de Euler Evanescente de Variedades De- terminantais}

No caso em que $V$ não é uma interseção completa, os autores em [12] definiram a característica de Euler evanescente singular como segue.

Definição 6.27. [12] Seja $V \subset \mathbb{C}^{p}$ uma variedade analítica complexa não necessariamente uma interseção completa e seja $X=F^{-1}(V)$. Suponha que $F: \mathbb{C}^{N} \rightarrow \mathbb{C}^{p}$ é transversal aos estratos de $V$ fora da origem, e considere $F^{\prime}$ uma estabilização de F. Então a característica de Euler evanescente singular de X é dada por:

$$
\tilde{\chi}(X)=\tilde{\chi}\left(F_{t}^{-1}(V)\right)=\chi\left(F_{t}^{-1}(V)\right)-1 \text {, em que } F_{t}=F^{\prime}(\cdot, t), t \neq 0 \text { pequeno. }
$$

Observação 6.28. [12] Se $V$ é uma interseção completa de codimensão igual a $k$ e $X=$ $F^{-1}(V)$ então:

$$
\tilde{\chi}(X)=(-1)^{N-k} \mu(X) .
$$

Lema 6.29. [12] Suponha que $F: \mathbb{C}^{N} \rightarrow \mathbb{C}^{p}$ é transversal a $V, W$ e $V \cap W$ fora da origem $0 \in \mathbb{C}^{N}$, então:

$$
\tilde{\chi}_{W \cup V}(F)=\tilde{\chi}_{W}(F)+\tilde{\chi}_{V}(F)-\tilde{\chi}_{W \cap V}(F)
$$

\begin{tabular}{lclr}
\hline $\mathcal{E}$ & Equação para $\mathcal{E}$ & $\mathcal{D}$ & Equação para $\mathcal{D}$ \\
\hline \hline $\mathcal{E}_{n}$ & $\prod_{k=1}^{n} \operatorname{det}\left(A^{(k)}\right) \cdot \prod_{k=1}^{n-1} \operatorname{det}\left(\hat{A}^{(k)}\right)$ & $\mathcal{D}_{n}$ & $\operatorname{det}\left(\hat{A}^{n-1}\right) \cdot \operatorname{det}(A)$ \\
$\mathcal{E}_{n-1, n}$ & $\prod_{k=1}^{n-1} \operatorname{det}\left(A^{(k)}\right) \cdot \prod_{k=1}^{n-1} \operatorname{det}\left(\hat{A}^{(k)}\right)$ & $\mathcal{D}_{n-1, n}$ & $\operatorname{det}\left(\hat{A}^{n-1}\right) \cdot \operatorname{det}\left(A^{n-1}\right)$ \\
\hline
\end{tabular}

Tabela 6.1: Tabela das equações definindo os divisores livres, $\mathcal{E}$ e as variedades determinantais $\mathcal{D}$. Se $A=\left(A_{i j}\right)$ então $\hat{A}$ denota a matriz obtida eliminando a primeira coluna da matriz $A$ e $A^{(k)}$ denota a submatriz $k \times k$ superior a esquerda de $A$.

Sejam $\mathcal{E}$ e $\mathcal{D}$ definidos na Tabela 6.1, no trabalho [12] se mostra que $\mathcal{E}$ é um divisor livre $H$ holonômico e que $\mathcal{D}$ tem completamento livre dado por:

$$
\begin{aligned}
\mathcal{E}_{n} & =\pi^{*} \mathcal{E}_{n-1, n} \cup \mathcal{D}_{n} \\
\mathcal{E}_{n-1, n} & =\pi^{*} \mathcal{E}_{n-1} \cup \mathcal{D}_{n-1, n}
\end{aligned}
$$

onde $\pi$ e $\pi^{\prime}$ são as projeções dadas por $\pi: M_{n} \rightarrow M_{n-1, n}$ e $\pi^{\prime}: M_{n-1, n} \rightarrow M_{n-1}$. 
As matrizes $2 \times 2, M_{2,2}$ podem ser representadas pelas coordenadas $\left(\begin{array}{ll}a & b \\ c & d\end{array}\right)$, o divisor $\mathcal{E}_{2}$ é definido por $a b(a d-b d)=0$ e $V\left(D_{2}\right)=V(a c-b d)=V(a b) \cup V(a d-b c)$. Então

Teorema 6.30. [12. Teorema 7.1] Sobre o espaço de germes transversais fora da origem as variedades associadas a $\mathcal{E}_{2}$, então

$$
\mu_{V\left(\mathcal{D}_{2}\right)}=\mu_{V\left(\mathcal{E}_{2}\right)}-\left(\left(\mu_{V(a)}+\mu_{V(a, c b)}\right)+\left(\mu_{V(b)}+\mu_{V(b, a d)}\right)\right)
$$

onde $\mu_{V\left(\mathcal{E}_{2}\right)}=\mathcal{K}_{\tilde{G}_{2}}$ - codim onde $\tilde{G}_{2}$ é o grupo de $B_{2} \times C_{2}$ preservando a equação ab $(a d-b c)=$ 0 .

Corolário 6.31. Com as hipótesis do teorema acima, a fórmula 6.3 pode ser reformulada por

$$
\mu_{V\left(\mathcal{D}_{2}\right)}=\mu_{V\left(\mathcal{E}_{2}\right)}+\mu_{V(b c)}-\mu_{V(a b c)}+\mu_{V(a d)}-\mu_{V(a b d)} .
$$

Todos os termos na parte dereita são números de Milnor singular de divisores livres holonômicos, que podem ser calculados usando o Teorema 6.25 .

Teorema 6.32. [12] Teorema 7.4] Para o espaço de germes transversais às variedades associadas a $\mathcal{E}_{2,3}$ fora da origem,

$$
\mu_{V_{1,3}}=\mu_{V\left(\mathcal{E}_{2,3}\right)}-\left(\mu_{V(a, b d e(d f-c e))}+\mu_{V(a)}\right)+\left(\mu_{V(a, e, b d f)}+\mu_{V(a, e)}\right)-\left(\mu_{V(b, a c e)}+\mu_{V(b)}\right) .
$$

Exemplo 6.33. Considere $F: \mathbb{C}^{5} \rightarrow M_{2,2}$ definido por

$$
\left(\begin{array}{cc}
u & v \\
w & x^{3}+y^{3}+u^{2}+v^{2}+w^{2}
\end{array}\right)
$$

Então $X=F^{-1}\left(M_{2,2}^{2}\right)=V\left(u\left(x^{3}+y^{3}+u^{2}+v^{2}+w^{2}\right)-v w\right)$ define uma hipersuperfície que não tem singularidade isolada em 0.

Usaremos a fórmula (6.4) para calcular $\mu_{V\left(\mathcal{D}_{2}\right)}(F)$. Pela tabela 6.4. temos que $\mathcal{D}_{2}=a d-b c$ $e \mathcal{E}_{2}=a b(a d-b c)$.

Como $(u, v, w) e(v, w)$ são submersões, então $\mu_{V(u, v, w)}(F)=\mu_{V(v, w)}(F)=0$.

$\mu_{V(a b(a d-b c))}=\mathcal{K}_{a b(a d-b c), e}-\operatorname{codim}(F)=16$, o Derlog $(a b(a d-b c))$ é gerado pelos vetores:

$$
\left(\begin{array}{cc}
u & 0 \\
-w & -2\left(x^{3}+y^{3}+u^{2}+v^{2}+w^{2}\right)
\end{array}\right),\left(\begin{array}{cc}
0 & v \\
-2 w & -\left(x^{3}+y^{3}+u^{2}+v^{2}+w^{2}\right)
\end{array}\right),\left(\begin{array}{ll}
0 & 0 \\
u & v
\end{array}\right) .
$$

Uma base para o espaço tangente estendido do grupo $\mathcal{K}_{H}, T \mathcal{K}_{H, e} \cdot F$, é dada pelos vetores 


$$
\left(\begin{array}{cc}
0 & 0 \\
0 & u^{2}
\end{array}\right),\left(\begin{array}{ll}
0 & 0 \\
0 & v
\end{array}\right),\left(\begin{array}{cc}
0 & 0 \\
0 & w^{2}
\end{array}\right),\left(\begin{array}{cc}
0 & 0 \\
0 & x^{2}
\end{array}\right),\left(\begin{array}{ll}
0 & 0 \\
0 & y^{2}
\end{array}\right),\left(\begin{array}{ll}
1 & 0 \\
0 & 0
\end{array}\right),\left(\begin{array}{ll}
0 & 1 \\
0 & 0
\end{array}\right),\left(\begin{array}{ll}
0 & 0 \\
1 & 0
\end{array}\right) .
$$

Derlog $(a d)$ e Derlog $(a b d)$ são gerados por $\left\{a \frac{\partial}{\partial a}-d \frac{\partial}{\partial d}, \frac{\partial}{\partial b}, \frac{\partial}{\partial c}\right\}$ e $\left\{a \frac{\partial}{\partial a}-d \frac{\partial}{\partial d}, b \frac{\partial}{\partial b}-d \frac{\partial}{\partial d}, \frac{\partial}{\partial c}\right\}$, $\operatorname{assim} \mathcal{K}_{a d, e}-\operatorname{codim}(F)=8 e \mathcal{K}_{a b d, e}-\operatorname{codim}(F)=16$.

Por Corolário 6.31, temos que $\mu_{V(a d-b c)}(F)=16+8-16=8$.

Teorema 6.34. [12. Teorema 8.1] Para um germe F transversal aos estratos de $M_{2,3}^{2}$, fora da origem, seja $X=F^{-1}\left(M_{2,3}^{2}\right)$ a EIDS correspondente. Então a característica de Euler evanescente singular de X é calculada por

$$
\tilde{\chi}(X)=(-1)^{N-1}\left(\mu_{V_{123}}(F)-\sum \mu_{V_{i j}}(F)+\sum_{i=1}^{3} \mu_{V_{i}}(F)\right),
$$

em que a primeira soma é sobre $\{i, j\}=\{1,2\},\{1,3\},\{2,3\}$ e $V_{123}=V_{1} \cup V_{2} \cup V_{3}$ é holonômico e pode ser calculado usando o Teorema 6.25 .

Se $F: \mathbb{C}^{N} \rightarrow M_{2,3}$ é um germe genérico de coposto igual a 1, Então

$$
F(x, y)=\sum_{i=1}^{5} x_{i} w_{i}+g(y) w_{0}
$$

onde $\left\{w_{1}, w_{2}, w_{3}, w_{4}, w_{5}\right\}$ é uma base para $W=d F(0)\left(\mathbb{C}^{N}\right), w_{0} \notin W$ e $g(y)$ define uma singularidade isolada sobre $\mathbb{C}^{N-5}$.

Corolário 6.35. [12] Se F é um germe genérico de coposto $1, N \geqslant 6$,

$$
\tilde{\chi}(X)=(-1)^{N-1} \mu_{V_{123}}(F)=(-1)^{N-1} \mu(g)
$$

Se gé quase-homogêneo, $\mu(g)$ é igual a $\tau(X)$.

\subsection{Obstrução de Euler de Variedades Determinantais}

Seja $X=F^{-1}\left(M_{m, n}^{t}\right)$ uma EIDS, definida pela função $F: \mathbb{C}^{N} \rightarrow M_{m, n}$. Se $N \leqslant(m-$ $t+3)(n-t+3)$ então $\Sigma X=F^{-1}\left(M_{m, n}^{t-1}\right)$ é uma variedade com singularidade isolada. Logo a variedade admite 3 estratos $\left\{V_{0}, V_{1}, V_{2}\right\}, V_{0}=\{0\}, V_{1}=\Sigma X \backslash\{0\}, V_{2}=X_{\text {reg. }}$. Esta é a classe de EIDS que consideramos nesta seção.

Para o cálculo da obstrução de Euler usamos a fórmula do Teorema 6.15, então temos $E u_{0}(X)=\chi\left(V_{0} \cap l^{-1}(r) \cap B_{\epsilon}\right) E u_{V_{0}}(X)+\chi\left(V_{1} \cap l^{-1}(r) \cap B_{\epsilon}\right) E u_{V_{1}}(X)+\chi\left(V_{2} \cap l^{-1}(r) \cap B_{\epsilon}\right) E u_{V_{2}}(X)$. 
Como $V_{0} \cap l^{-1}(r) \cap B_{\epsilon}=\emptyset$ então $\chi\left(V_{0} \cap l^{-1}(r) \cap B_{\epsilon}\right)=0$. Temos também $E u_{V_{2}}(X)=1$, então

$$
E u_{0}(X)=\chi\left(V_{1} \cap l^{-1}(r) \cap B_{\epsilon}\right)\left(E u_{V_{1}}(X)-1\right)+\chi\left(X \cap l^{-1}(r) \cap B_{\epsilon}\right)
$$

Note que $\chi\left(V_{1} \cap l^{-1}(r) \cap B_{\epsilon}\right)=\chi\left(\Sigma X \cap l^{-1}(r) \cap B_{\epsilon}\right)$ e por outro lado dado $a \in V_{1}$ existe um aberto $U_{a}$ contendo $a$ tal que $U_{a} \cong B_{\delta} \times c\left(L_{V_{1}}\right)$, onde a dimensão de $B_{\delta}$ é $\operatorname{dim} V_{1}=N-(m-$ $t+2)(n-t+2)$ e $c\left(L_{V_{1}}\right)$ é o cone do link complexo de $V_{1}$ em $X, L_{V_{1}}=X \cap \mathcal{N} \cap p^{-1}(s)$, com $\mathcal{N}$ transversal a $V_{1}$ em $a, \operatorname{codim} \mathcal{N}=\operatorname{dim} V_{1}$, então $E u_{V_{1}}(X)=E u_{a}\left(B_{\delta}\right) \times E u_{a}\left(c\left(L_{V_{1}}\right)\right)=$ $E u_{a}\left(c\left(L_{V_{1}}\right)\right)=\chi\left(L_{V_{1}}\right)=\chi\left(X \cap \mathcal{N} \cap p^{-1}(s)\right)$.

$X \cap \mathcal{N}$ é a suavização essencial de uma variedade determinantal de tipo $(m, n, t)$ em $\mathbb{C}^{r}$, onde $r=\operatorname{codim} V_{1}=(m-t+2)(n-t+2)$, por tanto com singularidade isolada.

Substituindo na equação (6.6), temos

$$
E u_{0}(X)=\chi\left(\Sigma X \cap l^{-1}(r) \cap B_{\epsilon}\right)\left(\chi\left(L_{V_{1}}\right)-1\right)+\chi\left(X \cap l^{-1}(r) \cap B_{\epsilon}\right) .
$$

Esta fórmula pode ser expressa em termos da característica de Euler evanescente singular da Definição 6.27

$$
E u_{0}(X)=\left(\tilde{\chi}\left(\Sigma X \cap l^{-1}(0) \cap B_{\epsilon}\right)+1\right)\left(\chi\left(L_{V_{1}}\right)-1\right)+\tilde{\chi}\left(X \cap l^{-1}(0) \cap B_{\epsilon}\right)+1 .
$$

Proposição 6.36. Seja $X=F^{-1}\left(M_{m, n}^{t}\right)$ uma EIDS, definida pela função $F: \mathbb{C}^{N} \rightarrow M_{m, n}$, se $N \leqslant(n-t+3)(m-t+3)$ e $\Sigma X$ é uma ICIS, então:

$$
E u_{0}(X)=\left((-1)^{\operatorname{dim}\left(\Sigma X \cap l^{-1}(0)\right)} \mu\left(\Sigma X \cap l^{-1}(0)\right)+1\right)\left(\chi\left(L_{V_{1}}\right)-1\right)+\tilde{\chi}\left(X \cap l^{-1}(0) \cap B_{\epsilon}\right)+1,
$$

onde l: $\mathbb{C}^{N} \rightarrow \mathbb{C}$ é uma projeção linear genérica, $L_{V_{1}}$ é o link complexo do estrato $V_{1}$ em $X$ e $B_{\epsilon}$ é a bola de raio $\epsilon$ em $\mathbb{C}^{N}$.

\section{Demonstração:}

Se $\Sigma X$ é uma interseção completa, então $\chi\left(\Sigma X \cap l^{-1}(r)\right)=1+(-1)^{\operatorname{dim}\left(\Sigma X \cap l^{-1}(0)\right)} \mu(\Sigma X \cap$ $\left.l^{-1}(0)\right)$ Substituindo esta fórmula em (6.8) obtemos o resultado.

O seguinte resultado mostra a existência de variedades determinantais cujo conjunto singular é uma ICIS.

Proposição 6.37. Seja $X=F^{-1}\left(M_{2, n}^{2}\right) \subset \mathbb{C}^{N}$ uma EIDS definida pela função $F: \mathbb{C}^{N} \rightarrow$ $M_{2, n}, n \geqslant 2$ então o conjunto singular de $X, \Sigma X \subset \mathbb{C}^{N}$ é uma ICIS.

Demonstração: Como $X=F^{-1}\left(M_{2, n}^{2}\right)$ é uma EIDS então $\Sigma X=F^{-1}\left(\Sigma M_{2, n}^{2}\right)=F^{-1}\left(M_{2, n}^{1}\right)$, como $M_{2, n}^{1}=\{0\}$, então $\Sigma X=F^{-1}(0)$ e $\operatorname{codim} \Sigma X=(2-2+2)(n-2+2)=2 \cdot n$, portanto $\Sigma X$ é uma interseção completa. 
Seja $x \in \Sigma X, x \neq 0$ então rank $F(x)=0$, pela definição de EIDS, temos que $F$ é transversal ao estrato corresponde, neste caso $F$ é transversal à $\{0\}$ fora da origem. Então $d F$ é uma submersão em $x \neq 0$, assim $\Sigma X$ é uma ICIS.

\subsubsection{Obstrução de Euler de IDS, caso $N<(n-t+2)(m-t+2)$}

Nesta seção trataremos as variedades IDS, definidas pela função $F: \mathbb{C}^{N} \rightarrow M_{m, n}$, tal que $N<(n-t+2)(m-t+2)$. Estas variedades admitem suavização e a obstrução de Euler foi estudada no trabalho [31], usando a característica de Euler evanescente e a multiplicidade da variedade.

Teorema 6.38. [31] Seja $X=F^{-1}\left(M_{m, n}^{t}\right)$ definida pela função $F: \mathbb{C}^{N} \rightarrow M_{m, n}$ com $N<$ $(n-t+2)(m-t+2)$, então

$$
E u_{0}(X)=1+(-1)^{d} \nu(X, 0)+(-1)^{d+1} m_{d}(X, 0)
$$

Demonstração: Pelo teorema 6.15 e pela definição da característica de Euler evanescente temos que $E u_{0}(X)=\chi\left(X \cap p^{-1}(t)\right)=(-1)^{d} \nu\left(X \cap p^{-1}(0)\right)+1$. Usando a fórmula de tipo Lê-Greuel (3.3), temos que $\nu(X)+\nu\left(X \cap p^{-1}(0)\right)=m_{d}(X)$. Então

$$
E u_{0}(X)+(-1)^{d} m_{d}(X)=1+(-1)^{d} \nu(X) .
$$

Então obtemos o resultado.

Exemplo 6.39. Seja $X=F^{-1}\left(M_{2,3}^{2}\right) \subset \mathbb{C}^{4}$ uma variedade determinantal com singularidade isolada, definida por $F$.

$$
\begin{aligned}
F: \mathbb{C}^{4} & \rightarrow M_{2,3} \\
(x, y, z, w) & \mapsto\left(\begin{array}{lll}
x & y & z \\
y & z & w
\end{array}\right)
\end{aligned}
$$

Por [31] temos que $m_{2}(X)=3, \nu(X)=1$, logo $E u_{0}(X)=-1$.

\subsection{Obstrução de Euler para as EIDS, definidas por $F: \mathbb{C}^{N} \rightarrow$ $M_{2,3}$}

O próximo resultado é para o caso $N=6$, neste caso a variedade determinantal não admite suavização, a fórmula do Teorema 6.38, dada acima não vale. 
Teorema 6.40. Seja $X=F^{-1}\left(M_{2,3}^{2}\right)$ definida pela função $F: \mathbb{C}^{N} \rightarrow M_{2,3}$ com $N=6$, então

$$
E u_{0}(X)=b_{2}\left(X \cap l^{-1}(r)\right)-b_{3}\left(X \cap l^{-1}(r)\right)+1 .
$$

Demonstração: $\quad$ Segue directamente pois $\chi\left(X \cap l^{-1}(r)\right)-1=\tilde{\chi}\left(X \cap l^{-1}(0)\right)=b_{2}(X \cap$ $\left.l^{-1}(r)\right)-b_{3}\left(X \cap l^{-1}(r)\right)$.

Nas tabelas 6.2, 6.3, calculamos a obstrução de Euler de variedades determinantais 4dimensionais em $\mathbb{C}^{6}$, classificadas em [18]. Usamos a Tabela 5 do trabalho [12] para obter os resultados.

\subsection{Obstrução de Euler de EIDS, caso $N \geqslant 7$}

Teorema 6.41. Seja $X \subset \mathbb{C}^{N}$ uma EIDS, definida pela função $F: \mathbb{C}^{N} \rightarrow M_{2,3}$, com $N \geqslant 7$. Então

$$
E u_{0}(X)=(-1)^{N-7} \mu\left(\Sigma X \cap l^{-1}(0)\right)+\tilde{\chi}\left(X \cap l^{-1}(0)\right)+2 .
$$

Demonstração: Como $X$ tem três estratos, $\left\{V_{0}, V_{1}, V_{2}\right\}, V_{0}=\{0\}, V_{1}=\Sigma \backslash\{0\}, V_{2}=X_{\text {reg }}$ e $V_{1}=F^{-1}\left(M_{2,3}^{1}\right) \backslash F^{-1}\left(M_{2,3}^{0}\right)$. Temos que $L_{V_{1}}=X \cap \mathcal{N} \cap p^{-1}(s)$, a variedade $X \cap \mathcal{N} \cong M_{2,3}^{2}$. Assim $\chi\left(L_{V_{1}}\right)-1=1$ pelo Teorema 6.16. Substituindo este valor na Proposição 6.36 temos o resultado.

Corolário 6.42. Com as hipóteses do Teorema 6.41 se F é de coposto igual a 1, então

$$
E u_{0}(X)=2
$$

Demonstração: Como $F$ tem coposto 1, então

$$
F(x, y)=\sum_{i=1}^{5} x_{i} w_{i}+g(y) w_{0}
$$

onde $\left\{w_{1}, w_{2}, w_{3}, w_{4}, w_{5}\right\}$ é uma base para $W=d_{0} F\left(\mathbb{C}^{N}\right), w_{0} \notin W$ e $g\left(y_{1}, y_{2}, \ldots, y_{N-5}\right)$ define uma singularidade isolada sobre $\mathbb{C}^{N-5}$. Suponha sem perda de generalidade que $l(x, y)=$ $y_{1}$ e considere $\tilde{g}\left(y_{2}, \ldots, y_{N-5}\right)=g\left(0, y_{2}, \ldots, y_{N-5}\right)$

Usando a fórmula do Corolário 6.35 para $X \cap l^{-1}(0) \subset \mathbb{C}^{N-1}$, temos que $\tilde{\chi}\left(X \cap l^{-1}(0)\right)=$ $(-1)^{N-2} \mu(\tilde{g})$.

Também temos que $\Sigma X \cap l^{-1}(0)=\left\{\left(0, \ldots, 0, y_{2}, \ldots, y_{N-5}\right) \mid \tilde{g}\left(y_{2}, \ldots, y_{N-5}\right)=0\right\}$ e portanto $\mu\left(\Sigma X \cap l^{-1}(0)\right)=\mu(\tilde{g})$. Substituindo na fórmula (6.10), temos que $E u_{0}(X)=2$.

Veremos dois exemplos de cálculo da obstrução de Euler usando Corolário 6.42 . 


\begin{tabular}{|c|c|c|c|c|}
\hline Tipo & $\begin{array}{c}\text { Apresentação } \\
\text { da Matriz }\end{array}$ & Condições & $\tau$ & $E u_{0}(X)$ \\
\hline$\Omega_{1}$ & $\left(\begin{array}{ccc}x & y & v \\
z & w & u\end{array}\right)$ & & 0 & 2 \\
\hline$\Omega_{k}$ & $\left(\begin{array}{ccc}x & y & v \\
z & w & x+u^{k}\end{array}\right)$ & $k \geqslant 2$ & $k-1$ & 2 \\
\hline$A_{k}^{\dagger}$ & $\left(\begin{array}{ccc}x & y & z \\
w & v & u^{2}+x^{k+1}+y^{2}\end{array}\right)$ & $k \geqslant 1$ & $k-2$ & 1 \\
\hline$D_{k}^{\dagger}$ & $\left(\begin{array}{ccc}x & y & z \\
w & v & u^{2}+x y^{2}+x^{k-1}\end{array}\right)$ & $k \geqslant 4$ & $k+2$ & -1 \\
\hline$E_{6}^{\dagger}$ & $\left(\begin{array}{ccc}x & y & z \\
w & v & u^{2}+y^{4}\end{array}\right)$ & & 8 & 0 \\
\hline$E_{7}^{\dagger}$ & $\left(\begin{array}{ccc}x & y & z \\
w & v & u^{2}+x^{3}+x y^{3}\end{array}\right)$ & & 9 & 0 \\
\hline$E_{8}^{\dagger}$ & $\left(\begin{array}{ccc}x & y & z \\
w & v & u^{2}+x^{3}+y^{5}\end{array}\right)$ & & 10 & 0 \\
\hline & $\left(\begin{array}{ccc}x & y & z \\
w & v & u x+y^{k}+u^{l}\end{array}\right)$ & $k \geqslant 2, l \geqslant 3$ & $k+l-1$ & $3-k$ \\
\hline & $\left(\begin{array}{ccc}x & y & z \\
w & v & x^{2}+y^{2}+u^{3}\end{array}\right)$ & & 6 & 1 \\
\hline$F_{q, r}^{\dagger}$ & $\left(\begin{array}{ccc}w & y & x \\
z & w+v u & y+v^{q}+u^{r}\end{array}\right)$ & $q, r \geqslant 2$ & $q+r$ & 2 \\
\hline$G_{5}^{\dagger}$ & $\left(\begin{array}{ccc}w & y & x \\
z & w+v^{2} & y+u^{3}\end{array}\right)$ & & 7 & 2 \\
\hline$G_{7}^{\dagger}$ & $\left(\begin{array}{ccc}w & y & x \\
z & w+v^{2} & y+u^{4}\end{array}\right)$ & & 10 & 2 \\
\hline$H_{q+3}^{\dagger}$ & $\left(\begin{array}{ccc}w & y & x \\
z & w+v^{2}+u^{q} & y+v u^{2}\end{array}\right)$ & $q \geqslant 3$ & $q+5$ & 2 \\
\hline
\end{tabular}

Tabela 6.2: Ostrução de Euler, $X \subset \mathbb{C}^{6}$ 


\begin{tabular}{|c|c|c|c|c|}
\hline Tipo & $\begin{array}{c}\text { Apresentação } \\
\text { da Matriz }\end{array}$ & Condições & $\tau$ & $E u_{0}(X)$ \\
\hline$I_{2 q-1}^{\dagger}$ & $\left(\begin{array}{ccc}w & y & v \\
z & w+v^{2}+u^{3} & y+u^{q}\end{array}\right)$ & $q \geqslant 4$ & $2 q+1$ & 2 \\
\hline \multirow[t]{7}{*}{$I_{2 r+2}^{\dagger}$} & $\left(\begin{array}{ccc}w & y & x \\
z & w+v^{2}+u^{3} & y+v u^{r}\end{array}\right)$ & $r \geqslant 3$ & $2 r+4$ & 2 \\
\hline & $\left(\begin{array}{ccc}w & y & x \\
z & w+v^{2} & u^{2}+y v\end{array}\right)$ & & 6 & 1 \\
\hline & $\left(\begin{array}{ccc}w & y & x \\
z & w+u v & u^{2}+y v+v^{k}\end{array}\right)$ & $k \geqslant 3$ & $k+4$ & 1 \\
\hline & $\left(\begin{array}{ccc}w & y & x \\
z & w+v^{k} & u^{2}+y v+v^{3}\end{array}\right)$ & $k \geqslant 3$ & $2 k+2$ & 1 \\
\hline & $\left(\begin{array}{ccc}w & y & x \\
z & w+u v^{k} & u^{2}+y v+v^{3}\end{array}\right)$ & $k \geqslant 2$ & $2 k+5$ & 1 \\
\hline & $\left(\begin{array}{ccc}w & y & x \\
z & w+v^{3} & u^{2}+y v\end{array}\right)$ & & 9 & 1 \\
\hline & $\left(\begin{array}{ccc}w & y & x \\
z & w+v^{k} & u^{2}+y^{2}=v^{3}\end{array}\right)$ & $k \geqslant 3$ & $2 k+3$ & 1 \\
\hline$H_{q+3}^{\dagger}$ & $\left(\begin{array}{ccc}w & y & x \\
z & w+u v^{k} & u^{2}+y^{2}+v^{3}\end{array}\right)$ & $k \geqslant 2$ & $2 k+6$ & 1 \\
\hline
\end{tabular}

Tabela 6.3: Obstrução de Euler, $X \subset \mathbb{C}^{6}$. 
Exemplo 6.43. Seja $X$ definido pela função $F$.

$$
\begin{aligned}
F: \mathbb{C}^{8} & \rightarrow \\
(x, y) & \mapsto\left(\begin{array}{ccc}
x_{1} & x_{2} & M_{2,3} \\
x_{4} & x_{5} & x_{1}+y_{1}^{2}-y_{2}^{2}+y_{3}^{2}
\end{array}\right)
\end{aligned}
$$

onde $x=\left(x_{1}, x_{2}, x_{3}, x_{4}, x_{5}\right)$ e $y=\left(y_{1}, y_{2}, y_{3}\right)$. A variedade $X$ é uma EIDS de dimensão 6 em $\mathbb{C}^{8}$. O conjunto singular é de dimensão 2 e é dado pelo conjunto

$$
\Sigma X=\left\{\left(0,0,0,0,0, y_{1}, y_{2}, y_{3}\right) \mid y_{1}^{2}-y_{2}^{2}+y_{3}^{2}=0\right\}
$$

Esta variedade tem três estratos $V_{0}=0, V_{1}=\Sigma X-\{0\}$ e $V_{2}=X_{\text {reg. }}$

Como o coposto de F é 1. Então pelo Corolário 6.42, temos:

$$
E u_{0}(X)=2
$$

Exemplo 6.44. Seja $X$ definido pela função $F$.

$$
\begin{aligned}
F: \mathbb{C}^{7} & \rightarrow M_{2,3} \\
(x, y) & \mapsto\left(\begin{array}{llc}
x_{1} & x_{2} & x_{3} \\
x_{4} & x_{5} & x_{1}+y_{1}^{2}-y_{2}^{2}
\end{array}\right)
\end{aligned}
$$

onde $x=\left(x_{1}, x_{2}, x_{3}, x_{4}, x_{5}\right)$ e $y=\left(y_{1}, y_{2}\right)$. A variedade $X$ é uma EIDS de dimensão 5 em $\mathbb{C}^{7}$. O conjunto singular é de dimensão 2 e é dado pelo conjunto

$$
\Sigma X=\left\{\left(0,0,0,0,0, y_{1}, y_{2}\right) \mid y_{1}^{2}-y_{2}^{2}=0\right\}
$$

Esta variedade tem três estratos $V_{0}=0, V_{1}=\Sigma X-\{0\}$ e $V_{2}=X_{\text {reg. }}$.

Como o coposto de F é 1. Então usando o Corolário 6.42 temos:

$$
E u_{0}(X)=2
$$




\section{Referências Bibliográficas}

[1] E. Arbarello, M. Cornalba, P. A. Griffiths, and J. Harris. Geometry of algebraic curves. Vol. I, volume 267 of Grundlehren der Mathematischen Wissenschaften [Fundamental Principles of Mathematical Sciences]. Springer-Verlag, New York, 1985.

[2] J.-P. Brasselet. Milnor classes via polar varieties. In Singularities in algebraic and analytic geometry (San Antonio, TX, 1999), volume 266 of Contemp. Math., pages 181-187. Amer. Math. Soc., Providence, RI, 2000.

[3] J.-P. Brasselet, D. T. Lê, and J. Seade. Euler obstruction and indices of vector fields. Topology, 39(6):1193-1208, 2000.

[4] J.-P. Brasselet et M.-H. Schwartz. Sur les classes de Chern d'un ensemble analytique complexe. In The Euler-Poincaré characteristic (French), volume 82 of Astérisque, pages 93147. Soc. Math. France, Paris, 1981.

[5] J.-P. Brasselet, J. Seade, and T. Suwa. Vector fields on singular varieties, volume 1987 of Lecture Notes in Mathematics. Springer-Verlag, Berlin, 2009.

[6] J. Briançon, A. Galligo, et M. Granger. Déformations équisingulières des germes de courbes gauches réduites. Mém. Soc. Math. France (N.S.), (1):69, 1980/81.

[7] R.-O. Buchweitz and G.-M. Greuel. The Milnor number and deformations of complex curve singularities. Invent. Math., 58(3):241-281, 1980.

[8] E. M. Chirka. Complex analytic sets, volume 46 of Mathematics and its Applications (Soviet Series). Kluwer Academic Publishers Group, Dordrecht, 1989. Translated from the Russian by R. A. M. Hoksbergen.

[9] J. Damon. The unfolding and determinacy theorems for subgroups of $A$ and $K$. Mem. Amer. Math. Soc., 50(306):x+88, 1984. 
[10] J. Damon. Higher multiplicities and almost free divisors and complete intersections. Mem. Amer. Math. Soc., 123(589):x+113, 1996.

[11] J. Damon and D. Mond. A-codimension and the vanishing topology of discriminants. Invent. Math., 106(2):217-242, 1991.

[12] J. Damon and B. Pike. Solvable groups, free divisors and nonisolated matrix singularities II: Vanishing topology. Geom. Topol., 18(2):911-962, 2014.

[13] L. R. Dias. Regularity at infinity and global fibrations of real algebraic maps. $\mathrm{PhD}$ thesis, ICMC-USP, 2013.

[14] W. Ebeling and S. M. Gusein-Zade. Radial index and Euler obstruction of a 1-form on a singular variety. Geom. Dedicata, 113:231-241, 2005.

[15] D. Einsenbud. Commutative Algebra with a view towards Algebraic Geometry. SpringerVerlag, 1995.

[16] A. Frühbis-Krüger. Classification of simple space curve singularities. Comm. Algebra, 27(8):3993-4013, 1999.

[17] A. Frühbis-Krüger. Moduli spaces for space curve singularities. PhD thesis, Universität Kaiserslautern, 2000.

[18] A. Frühbis-Krüger and A. Neumer. Simple Cohen-Macaulay codimension 2 singularities. Comm. Algebra, 38(2):454-495, 2010.

[19] W. Fulton. Algebraic curves. An introduction to algebraic geometry. W. A. Benjamin, Inc., New York-Amsterdam, 1969. Notes written with the collaboration of Richard Weiss, Mathematics Lecture Notes Series.

[20] T. Gaffney. Polar multiplicities and equisingularity of map germs. Topology, 32(1):185223, 1993.

[21] T. Gaffney. Aureoles and integral closure of modules. In Stratifications, singularities and differential equations, II (Marseille, 1990; Honolulu, HI, 1990), volume 55 of Travaux en Cours, pages 55-62. Hermann, Paris, 1997.

[22] G.-M. Greuel and J. Steenbrink. On the topology of smoothable singularities. In Singularities, Part 1 (Arcata, Calif., 1981), volume 40 of Proc. Sympos. Pure Math., pages 535-545. Amer. Math. Soc., Providence, R.I., 1983. 
[23] S. M. Guse ̌n-Zade and W. Èbeling. On the indices of 1-forms on determinantal singularities. Tr. Mat. Inst. Steklova, 267(Osobennosti i Prilozheniya):119-131, 2009.

[24] R. Hartshorne. Book Review: Algebraic geometry I: Complex projective varieties. Bull. Amer. Math. Soc. (N.S.), 1(3):513-515, 1979.

[25] J.-P. G. Henry et D. T. Lê. Limites d'espaces tangents. In Fonctions de plusieurs variables complexes, II (Sém. François Norguet, 1974-1975), pages 251-265. Lecture Notes in Math., Vol. 482. Springer, Berlin, 1975.

[26] D. T. Lê. Generic sections of singularities. In Singularity theory, pages 677-682. World Sci. Publ., Hackensack, NJ, 2007.

[27] D. T. Lê et B. Teissier. Variétés polaires locales et classes de Chern des variétés singulières. Ann. of Math. (2), 114(3):457-491, 1981.

[28] D. T. Lê et B. Teissier. Limites d'espaces tangents en géométrie analytique. Comment. Math. Helv., 63(4):540-578, 1988.

[29] R. D. MacPherson. Chern classes for singular algebraic varieties. Ann. of Math. (2), 100:423-432, 1974.

[30] W. L. Marar, J. A. Montaldi, and M. A. S. Ruas. Multiplicities of zero-schemes in quasihomogeneous corank-1 singularities $\mathbf{C}^{n} \rightarrow \mathbf{C}^{n}$. In Singularity theory (Liverpool, 1996), volume 263 of London Math. Soc. Lecture Note Ser., pages 353-367. Cambridge Univ. Press, Cambridge, 1999.

[31] J. Nuño Ballesteros, B. Oréfice-Okamoto, and J. Tomazella. The vanishing Euler characteristic of an isolated determinantal singularity. Israel Journal of Mathematics, 197(1):475495, 2013.

[32] J. J. Nuño-Ballesteros and J. N. Tomazella. The Milnor number of a function on a space curve germ. Bull. Lond. Math. Soc., 40(1):129-138, 2008.

[33] B. Oréfice. O número de Milnor de uma singularidade isolada. PhD thesis, UFSCAR, 2011.

[34] M. S. Pereira. Variedades determinantais e singularidades de matrizes. $\mathrm{PhD}$ thesis, ICMC, 2010.

[35] M. S. Pereira and M. A. S. Ruas. Codimension two determinantal varieties with isolated singularities. Math. Scandinavica, to appear. 
[36] B. Pike. Singular Milnor numbers of non-isolated singularities. PhD thesis, University of North Carolina, 2010.

[37] D. Rees. A-transforms of local rings and a theorem on multiplicities of ideals. Proc. Cambridge Philos. Soc., 57:8-17, 1961.

[38] J. Snoussi. Limites d'espaces tangents à une surface normale. Comment. Math. Helv., 76(1):61-88, 2001.

[39] B. Teissier. Variétés polaires. II. Multiplicités polaires, sections planes, et conditions de Whitney. In Algebraic geometry (La Rábida, 1981), volume 961 of Lecture Notes in Math., pages 314-491. Springer, Berlin, 1982.

[40] J. Wahl. Smoothings of normal surface singularities. Topology, 20(3):219-246, 1981. 